Supporting Information

\title{
Regioselective [5,5]-sigmatropic rearrangement reactions of aryl hydrazides
}

Hong-Min Kang, Young-Kwan Lim, In-Ji Shin, Hee-Yeon Kim and Cheon-Gyu Cho*

Department of Chemistry, Hanyang University, Seoul, Korea 133-791. 
Materials and Methods. Reactions were carried out in oven or flame-dried glassware under an argon atmosphere, unless otherwise noted. All solvents were reagent grade. Diethyl ether $\left(\mathrm{Et}_{2} \mathrm{O}\right)$ and tetrahydrofuran (THF) were freshly distilled from sodium/benzophenone under argon. $n$-Butyllithium and $t$-butyllithium were purchased from Aldrich. Reactions were magnetically stirred and monitored by thin layer chromatography (TLC) with $0.25 \mathrm{~mm}$ E. Merck pre-coated silica gel plates. Yields refer to chromatographically and spectroscopically pure compounds, unless otherwise stated. Infrared spectra were recorded on a Jasco Model FT/IR480 Plus spectrometer. Proton and carbon-13 NMR spectra were recorded on a Varian 400 MHz spectrometer. Chemical shifts are reported relative to chloroform $(\delta=7.26)$ or TMS $(\delta=$ $0.00)$ for ${ }^{1} \mathrm{H}$ NMR and chloroform $(\delta=77.0)$ for ${ }^{13} \mathrm{C}$ NMR. High resolution mass spectra were measured at the Korean Basic Research Center, Seoul, Korea.

\section{Experimental Procedures}

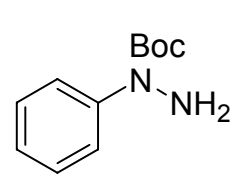

N-Phenyl-hydrazinecarboxylic aicd tert-butyl ester (4a)

${ }^{1} \mathrm{H}$ NMR $\left(400 \mathrm{MHz}, \mathrm{CDCl}_{3}\right) \delta 7.42(\mathrm{~d}, \mathrm{~J}=8.0 \mathrm{~Hz}, 2 \mathrm{H}), 7.28-7.22(\mathrm{~m}, 2 \mathrm{H}), 7.08-7.04(\mathrm{~m}, 1 \mathrm{H})$, 4.43 (bs, 2H), 1.48 (s, 9H); Lim, Y.-K.; Choi, S.; Park, K.-B.; Cho, C.-G. J. Org. Chem. 2004, 69, 2603.

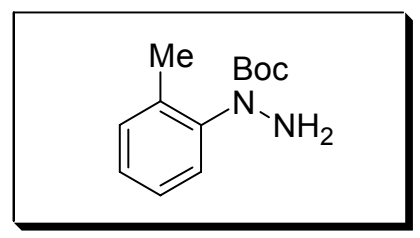

N-o-Tolyl-hydrazinecarboxylic acid tert-butyl ester (4b)

${ }^{1} \mathrm{H}$ NMR (400 MHz, $\left.\mathrm{CDCl}_{3}\right) 7.19$ - $7.16(\mathrm{~m}, 4 \mathrm{H}), 4.46$ (bs, 2H), 2.25 (s, 3H), 1.39 (s, 9H); Wolter, M.; Klapars, A.; Buchwald, S. L. Org. Lett, 2001, 3, 3803. 


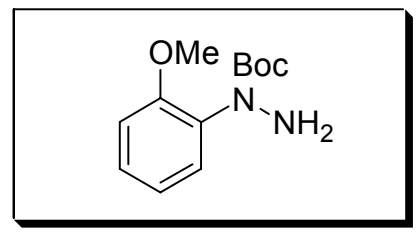

N-(2-Methoxy-phenyl)-hydrazinecarboxylic acid tert-butyl ester (4c)

${ }^{1} \mathrm{H}$ NMR $\left(400 \mathrm{MHz}, \mathrm{CDCl}_{3}\right) \delta$ 7.24-7.18 (m, 2H), 6.92 - $6.88(\mathrm{~m}, 2 \mathrm{H}), 4.48(\mathrm{~s}, 2 \mathrm{H}), 3.83(\mathrm{~s}, 3 \mathrm{H})$, 1.40 (s, 9H); Lim, Y.-K.; Choi, S.; Park, K.-B.; Cho, C.-G. J. Org. Chem. 2004, 69, 2603.

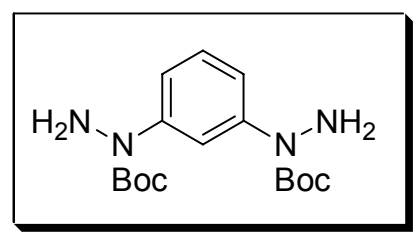

$N$-[3-(N-tert-Butoxycarbonyl-hydrazino)-phenyl]-hydrazinecarboxylic acid tert-butyl ester (8)

To a sealed tube were charged $2.00 \mathrm{~g}(6.06 \mathrm{mmol})$ of 1,3-diiodobenzene, $1.15 \mathrm{~g}(6.06 \mathrm{mmol})$ of Cul, $1.09 \mathrm{~g}$ (6.06 mmol) of 1,10-phenanthroline, $5.92 \mathrm{~g}$ (18.18 mmol) of $\mathrm{Cs}_{2} \mathrm{CO}_{3}, 2.40 \mathrm{~g} \mathrm{(18.18}$ $\mathrm{mmol}$ ) of tert-butyl hydrazate, and $5 \mathrm{~mL}$ anhydrous DMF at rt. The reaction mixture was degassed, charged with $\operatorname{Ar}$ gas and heated to $80^{\circ} \mathrm{C}$. After $6 \mathrm{~h}$, The resulting mixture was directly purified by column chromatography (hexane:EtOAc $=5: 1$ ) to afford $1.67 \mathrm{~g}$ of product in $82 \%$ yields. ${ }^{1} \mathrm{H}$ NMR (400 MHz, $\left.\mathrm{CDCl}_{3}\right) \delta 7.67$ (s, 1H), 7.21 (bs, 3H), 4.43 (bs, $\left.4 \mathrm{H}\right), 1.51$ (s, $\left.18 \mathrm{H}\right) ;{ }^{13} \mathrm{C}$ NMR $\left(100 \mathrm{MHz}, \mathrm{CDCl}_{3}\right) \delta 155.0,143.0,127.5,119.5,118.2,81.8$, 28.3; FT-IR $\left(\mathrm{CHCl}_{3}\right)$ 3339, 2977, 2930, 1771, 1699, 1602, 1488, $1368 \mathrm{~cm}^{-1}$; HRMS Calcd for $\mathrm{C}_{16} \mathrm{H}_{26} \mathrm{~N}_{4} \mathrm{NaO}_{4}\left(\mathrm{M}+\mathrm{Na}^{+}\right)$: 361.1852 , found 361.1862 .

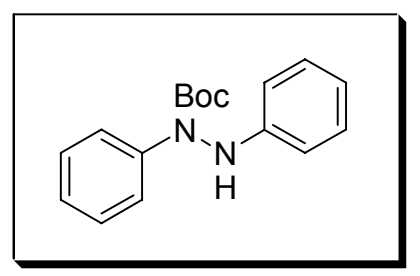

\section{$N, N$ '-Diphenyl-hydrazinecarboxylic acid tert-butyl ester (6a)}

To a round flask were charged $329 \mathrm{mg}(1.58 \mathrm{mmol})$ of $\mathrm{N}$-phenyl-hydrazine carboxylic acid tertbutyl ester, $267 \mathrm{mg}$ (1.32 mmol) of iodobenzene, $5 \mathrm{~mol} \%$ of $\mathrm{Pd}(\mathrm{OAc})_{2}, 5 \mathrm{~mol} \%$ of $\mathrm{P}(t-\mathrm{Bu})_{3} \cdot \mathrm{BF}_{4}$, $602 \mathrm{mg}(1.85 \mathrm{mmol})$ of $\mathrm{Cs}_{2} \mathrm{CO}_{3}$, and $5 \mathrm{~mL}$ of anhydrous toluene at rt. The reaction mixture was 
stirred for $30 \mathrm{~min}$ and refluxed for $2 \mathrm{~h}$. The resulting mixture was directly purified by column chromatography (hexane:EtOAc $=20: 1)$ to afford $360 \mathrm{mg}$ of product in $96 \%$ yield. ${ }^{1} \mathrm{H}$ NMR $(400$ $\left.\mathrm{MHz}, \mathrm{CDCl}_{3}\right) \delta 7.60(\mathrm{~d}, \mathrm{~J}=7.6 \mathrm{~Hz}, 2 \mathrm{H}), 7.35-7.30(\mathrm{~m}, 2 \mathrm{H}), 7.27-7.21(\mathrm{~m}, 2 \mathrm{H}), 7.13-7.10(\mathrm{~m}$, $1 \mathrm{H}), 6.93-6.88(\mathrm{~m}, 1 \mathrm{H}), 6.81(\mathrm{~d}, \mathrm{~J}=7.2 \mathrm{~Hz}, 2 \mathrm{H}), 6.38(\mathrm{bs}, 1 \mathrm{H}), 1.38(\mathrm{~s}, 9 \mathrm{H}) ;{ }^{13} \mathrm{C} \mathrm{NMR}(100$ $\left.\mathrm{MHz}, \mathrm{CDCl}_{3}\right) \delta 153.9,148.3,142.8,129.1,128.4,124.4,121.5,120.9,112.9,82.2$, 28.0; FT-IR $\left(\mathrm{CHCl}_{3}\right)$ 3354, 3055, 2983, 2933, 1710, 1601, 1496, 1369, $1265 \mathrm{~cm}^{-1}$; HRMS Calcd for $\mathrm{C}_{17} \mathrm{H}_{20} \mathrm{~N}_{2} \mathrm{O}_{2}\left(\mathrm{M}^{+}\right): 284.1525$, found 284.1529 .

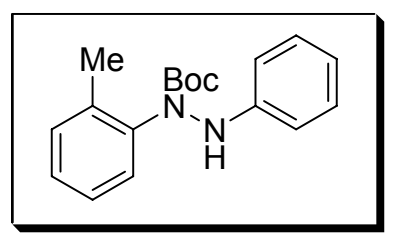

\section{N'-Phenyl-N-o-tolyl-hydrazinecarboxylic acid tert-butyl ester (6b)}

${ }^{1} \mathrm{H}$ NMR $\left(400 \mathrm{MHz}, \mathrm{CDCl}_{3}\right) \delta 7.41-7.39(\mathrm{~m}, 1 \mathrm{H}), 7.26-7.16(\mathrm{~m}, 5 \mathrm{H}), 6.94-6.88(\mathrm{~m}, 3 \mathrm{H}), 6.40$ (bs, 1H), 2.35 (s, 3H), 1.37 (s, 9H); ${ }^{13} \mathrm{C}$ NMR (100 MHz, $\left.\mathrm{CDCl}_{3}\right) \delta$ 155.8, 148.7, 142.2, 135.2, 131.6, 129.8, 127.9, 127.4, 127.1, 121.7, 114.3, 82.4, 28.8, 19.1; FT-IR $\left(\mathrm{CHCl}_{3}\right) 3325,3057$, 2978, 2928, 1701, 1601, 1497, 1369, 1333, 1240, $1154 \mathrm{~cm}^{-1}$; HRMS Calcd for $\mathrm{C}_{18} \mathrm{H}_{22} \mathrm{~N}_{2} \mathrm{O}_{2}\left(\mathrm{M}^{+}\right)$: 298.1681, found 298.1693.

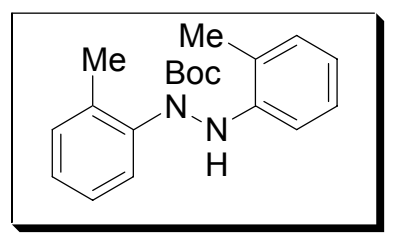

N,N'-Di-o-tolyl-hydrazinecarboxylic acid tert-butyl ester (6c)

${ }^{1} \mathrm{H}$ NMR $\left(400 \mathrm{MHz}, \mathrm{CDCl}_{3}\right) \delta 7.44-7.42(\mathrm{~m}, 1 \mathrm{H}), 7.23-7.13(\mathrm{~m}, 4 \mathrm{H}), 7.08-7.05$ (d, J= $8.8 \mathrm{~Hz}$, $2 \mathrm{H}), 6.84-6.80(\mathrm{td}, \mathrm{J}=7.3,1.1 \mathrm{~Hz}, 1 \mathrm{H}), 6.34(\mathrm{bs}, 1 \mathrm{H}), 2.38(\mathrm{~s}, 3 \mathrm{H}), 2.22(\mathrm{~s}, 3 \mathrm{H}), 1.37(\mathrm{~s}, 9 \mathrm{H})$; ${ }^{13} \mathrm{C}$ NMR $\left(100 \mathrm{MHz}, \mathrm{CDCl}_{3}\right) \delta 155.7,146.3,142.1,135.2,131.6,131.1,127.8,127.5,127.4$, 127.0, 123.2, 121.3, 112.8, 82.4, 28.7, 19.3, 17.7; FT-IR $\left(\mathrm{CHCl}_{3}\right)$ 3301, 3022, 2977, 2930, 1702 , 1607, 1590, 1482, 1457, 1368, 1332, 1247, 1155; HRMS Calcd for $\mathrm{C}_{19} \mathrm{H}_{24} \mathrm{~N}_{2} \mathrm{O}_{2}\left(\mathrm{M}^{+}\right)$: 312.1838 , found 312.1831 . 


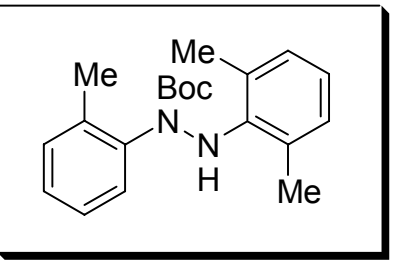

$N^{\prime}$-(2,6-Dimethyl-phenyl)-N-o-tolyl-hydrazinecarboxylic acid tert-butyl ester (6d)

${ }^{1} \mathrm{H}$ NMR $\left(400 \mathrm{MHz}, \mathrm{CDCl}_{3}\right) \delta 7.71-7.10(\mathrm{~m}, 2 \mathrm{H}), 6.99-6.95(\mathrm{~m}, 1 \mathrm{H}), 6.91-6.83(\mathrm{~m}, 3 \mathrm{H})$, $6.67(\mathrm{dd}, \mathrm{J}=1.2 \mathrm{~Hz}, 1 \mathrm{H}), 5.76(\mathrm{~s}, 1 \mathrm{H}), 2.24(\mathrm{~s}, 3 \mathrm{H}), 2.12(\mathrm{~s}, 6 \mathrm{H}), 1.45(\mathrm{~s}, 9 \mathrm{H}) ;{ }^{13} \mathrm{C}$ NMR $(100$ $\left.\mathrm{MHz}, \mathrm{CDCl}_{3}\right) \delta 155.4,141.8,141.0,135.1,131.1,130.7,128.8,128.0,127.9,126.3,124.0,80.8$, 28.2, 17.9, 17.4; FT-IR $\left(\mathrm{CHCl}_{3}\right) 3257,2976,2927,2568,1716,1684,1521,1507 \mathrm{~cm}^{-1}$; HRMS Calcd for $\mathrm{C}_{20} \mathrm{H}_{26} \mathrm{~N}_{2} \mathrm{NaO}_{2}\left(\mathrm{M}+\mathrm{Na}^{+}\right): 349.1892$, found 349.1879.

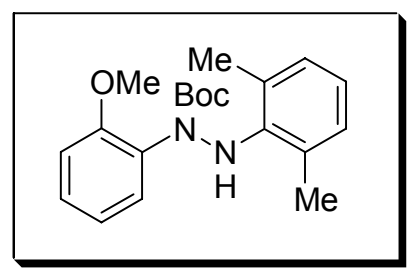

$N^{\prime}$-(2,6-Dimethyl-phenyl)-N-(2-methoxy-phenyl)-hydrazinecarboxylic acid tert-butyl ester (6e) ${ }^{1} \mathrm{H}$ NMR $\left(400 \mathrm{MHz}, \mathrm{CDCl}_{3}\right) \delta 7.20-7.16(\mathrm{~m}, 1 \mathrm{H}), 6.91-6.86(\mathrm{~m}, 3 \mathrm{H}), 6.80-6.70(\mathrm{~m}, 3 \mathrm{H})$, 6.30 (bs, 1H), 3.92 (s, 3H), $2.18(\mathrm{~s}, 6 \mathrm{H}), 1.43(\mathrm{~s}, 9 \mathrm{H}) ;{ }^{13} \mathrm{C} \mathrm{NMR}\left(100 \mathrm{MHz}, \mathrm{CDCl}_{3}\right) \delta$ 157.0, 155.0, 143.0, 131.7, 130.2, 129.8, 129.5, 129.4, 123.4, 121.0, 111.8, 81.4, 56.1, 28.9, 18.6; FTIR $\left(\mathrm{CHCl}_{3}\right) 3278,2975,2930,1730,1700,1533,1493,1458,1248 \mathrm{~cm}^{-1}$; HRMS Calcd for $\mathrm{C}_{20} \mathrm{H}_{26} \mathrm{~N}_{2} \mathrm{NaO}_{3}\left(\mathrm{M}+\mathrm{Na}^{+}\right): 365.1841$, found 365.1837.

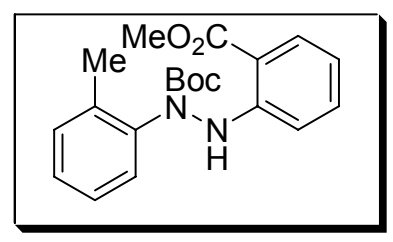

\section{2-(N'-tert-Butoxycarbonyl-N'-o-tolyl-hydrazino)-benzoic acid methyl ester (6f)}

${ }^{1} \mathrm{H}$ NMR $\left(400 \mathrm{MHz}, \mathrm{CDCl}_{3}\right) \delta 7.93-7.91$ (dd, $\left.\mathrm{J}=8.1,1.5 \mathrm{~Hz}, 1 \mathrm{H}\right), 7.46-7.40(\mathrm{~m}, 2 \mathrm{H}), 7.25-$ $7.14(\mathrm{~m}, 4 \mathrm{H}), 6.81-6.77\left(\mathrm{td}, J_{1}=7.3 \mathrm{~Hz}, J_{2}=1.1 \mathrm{~Hz}, 1 \mathrm{H}\right), 3.87(\mathrm{~s}, 3 \mathrm{H}), 2.41(\mathrm{~s}, 3 \mathrm{H}), 1.38(\mathrm{~s}$, $9 \mathrm{H}) ;{ }^{13} \mathrm{C}$ NMR $\left(100 \mathrm{MHz}, \mathrm{CDCl}_{3}\right) \delta 169.1,155.5,151.8 .141 .9,135.4,135.1,132.0,131.8,128.0$, 127.3, 127.1, 118.8, 113.3, 112.1, 82.3, 52.5, 28.7, 19.1; FT-IR $\left(\mathrm{CHCl}_{3}\right)$ 3328, 2977, 2929, 1718, $1693,1606,1586,1498,1457,1438,1368,1331,1256,1160$; HRMS Calcd for $\mathrm{C}_{20} \mathrm{H}_{24} \mathrm{~N}_{2} \mathrm{NaO}_{4}$ $\left(\mathrm{M}+\mathrm{Na}^{+}\right): 379.1634$, found 379.1628 . 


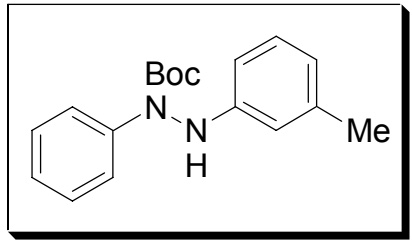

$N^{\prime}$-(1,3-Dimethyl-buta-1,3-dienyl)- $N$-phenyl-hydrazinecarboxylic acid tert-butyl ester (13a) ${ }^{1} \mathrm{H}$ NMR $\left(400 \mathrm{MHz}, \mathrm{CDCl}_{3}\right) \delta 7.60-7.58(\mathrm{~m}, 2 \mathrm{H}), 7.33-7.29(\mathrm{~m}, 2 \mathrm{H}), 7.12-7.08(\mathrm{~m}, 2 \mathrm{H}), 6.72$ $-6.70\left(\mathrm{~d}, \mathrm{~J}=7.3 \mathrm{~Hz}, 1 \mathrm{H}, 6.62-6.60(\mathrm{~m}, 2 \mathrm{H}), 6.34\right.$ (bs, 1H), 2.27 (s, 3H), 1.37 (s, 9H); ${ }^{13} \mathrm{C} \mathrm{NMR}$ $\left(100 \mathrm{MHz}, \mathrm{CDCl}_{3}\right) \delta 154.6,149.0,143.6,139.7,129.7,129.1,125.1,122.5,122.2,114.2,110.8$, 82.9, 28.7, 22.2; FT-IR $\left(\mathrm{CHCl}_{3}\right) 3338,3042,2978,2929,1714,1611,1596,1494,1455,1392$, 1368, 1335, 1307, 1251, 1156; HRMS Calcd for $\mathrm{C}_{18} \mathrm{H}_{22} \mathrm{~N}_{2} \mathrm{O}_{2}\left(\mathrm{M}^{+}\right)$: 298.1681, found 298.1687.

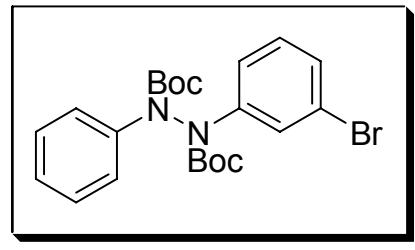

\section{Di-tert-butyl 1-(3-bromophenyl)-2-phenylhydrazine-1,2-dicarboxylate (13b)}

${ }^{1} \mathrm{H}$ NMR $\left(400 \mathrm{MHz}, \mathrm{CDCl}_{3}\right) \delta 7.66$ (bs, $\left.1 \mathrm{H}\right), 7.37-7.26-7.40(\mathrm{~m}, 6 \mathrm{H}), 7.18-7.14(\mathrm{t}, \mathrm{J}=8.1 \mathrm{~Hz}$, 2H), 1.52 (s, 18H); $\left.{ }^{13} \mathrm{C} \mathrm{NMR} \mathrm{(100} \mathrm{MHz,} \mathrm{CDCl}_{3}\right)$ 153.6, 153.4, 143.1, 141.5, 135.0, 130.5, 129.3, 129.0, 126.4, 122.9 (bs), 122.7, 121.1 (bs), 83.5, 83.3, 28.8, 28.8; FT-IR $\left(\mathrm{CHCl}_{3}\right)$ 2979, 2932, 1726, 1590, 1573, 1494, 1477, 1456, 1369, 1341, 1320, 1255, 1153; HRMS Calcd for $\mathrm{C}_{22} \mathrm{H}_{27} \mathrm{BrN}_{2} \mathrm{O}_{4}\left(\mathrm{M}^{+}\right): 462.1154$, found 462.1148 .

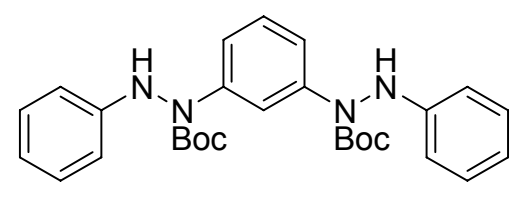

Di-tert-butyl 1,1'-(1,3-phenylene)bis(2-phenylhydrazinecarboxylate) (9a)

${ }^{1} \mathrm{H}$ NMR $\left(400 \mathrm{MHz}, \mathrm{CDCl}_{3}\right) \delta 7.89(\mathrm{~s}, 1 \mathrm{H}), 7.38-7.36(\mathrm{~m}, 2 \mathrm{H}), 7.28-7.20(\mathrm{~m}, 5 \mathrm{H}), 6.91-6.88$ $(\mathrm{t}, J=7.3 \mathrm{~Hz}, 2 \mathrm{H}), 6.81-6.79(\mathrm{~d}, \mathrm{~J}=7.7 \mathrm{~Hz}, 4 \mathrm{H}), 6.37(\mathrm{bs}, 2 \mathrm{H}), 1.35(\mathrm{~s}, 18 \mathrm{H}) ;{ }^{13} \mathrm{C}$ NMR $(100$ $\left.\mathrm{MHz}, \mathrm{CDCl}_{3}\right) \delta 153.6,148.0 .143 .0,129.0,128.1,120.9,117.8,115.2,113.0,82.3,28.1 ; \mathrm{FT}-\mathrm{IR}$ $\left(\mathrm{CHCl}_{3}\right)$ 3336, 2982, 2928, 2857, 1709, 1601, 1497, 1455, 1369, 1322, 1304, 1254, 1151; HRMS Calcd for $\mathrm{C}_{28} \mathrm{H}_{34} \mathrm{~N}_{4} \mathrm{NaO}_{4}\left(\mathrm{M}+\mathrm{Na}^{+}\right): 513.2478$, found 513.2487. 


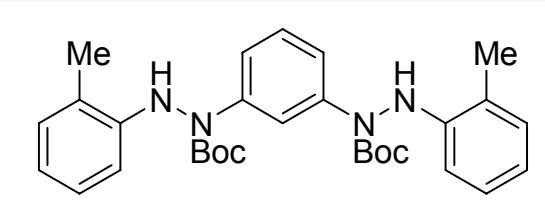

Di-tert-butyl 1,1'-(1,3-phenylene)bis(2-o-tolylhydrazinecarboxylate) (9b)

${ }^{1} \mathrm{H}$ NMR $\left(400 \mathrm{MHz}, \mathrm{CDCl}_{3}\right) \delta 7.90(\mathrm{~s}, 1 \mathrm{H}), 7.38-7.35$ (dd, $\left.\mathrm{J}_{1}=8.2 \mathrm{~Hz}, \mathrm{~J}_{2}=1.8 \mathrm{~Hz}, 2 \mathrm{H}\right), 7.23-$ $7.21(\mathrm{~m}, 1 \mathrm{H}), 7.07-7.04(\mathrm{~m}, 4 \mathrm{H}), 6.82-6.75(\mathrm{~m}, 4 \mathrm{H}), 6.35(\mathrm{bs}, 2 \mathrm{H}), 2.23(\mathrm{~s}, 6 \mathrm{H}), 1.33(\mathrm{~s}$, $18 \mathrm{H}) ;{ }^{13} \mathrm{C}$ NMR $\left(100 \mathrm{MHz}, \mathrm{CDCl}_{3}\right) \delta 153.5,145.7,142.9,130.1,128.1,126.9,122.0,120.5$, 117.7, 115.5, 111.5, 82.2, 28.1, 17.1; FT-IR $\left(\mathrm{CHCl}_{3}\right)$ 3354, 2978, 2928, 2853, 1712, 1598, 1480 , 1455, 1365, 1329, 1301, 1251, 1154; HRMS Calcd for $\mathrm{C}_{30} \mathrm{H}_{38} \mathrm{~N}_{4} \mathrm{NaO}_{4}\left(\mathrm{M}+\mathrm{Na}^{+}\right)$: 541.2791, found 541.2801 .

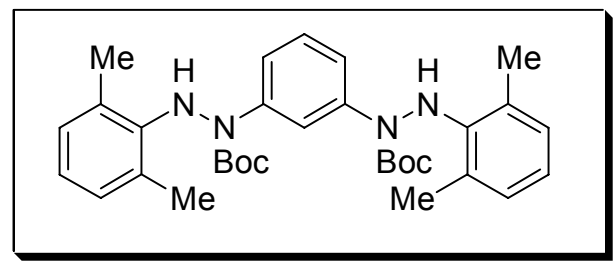

Di-tert-butyl 1,1'-(1,3-phenylene)bis(2-(2,6-dimethylphenyl)hydrazinecarboxylate) (9c)

${ }^{1} \mathrm{H}$ NMR $\left(400 \mathrm{MHz}, \mathrm{CDCl}_{3}\right) \delta 7.90(\mathrm{t}, \mathrm{J}=2.4 \mathrm{~Hz}, 1 \mathrm{H}), 7.40(\mathrm{dd}, \mathrm{J}=2.0 \mathrm{~Hz}, 2 \mathrm{H}), 7.22(\mathrm{t}, \mathrm{J}=4.0$ $\mathrm{Hz}, 1 \mathrm{H}$ ), 6.88 (d, J = 7.2 Hz, 4H), $7.74(\mathrm{t}, \mathrm{J}=3.6 \mathrm{~Hz}, 2 \mathrm{H}), 6.16(\mathrm{~s}, 2 \mathrm{H}), 2.17$ (s, $12 \mathrm{H}), 1.31$ (s, $18 \mathrm{H}) ;{ }^{13} \mathrm{C}$ NMR $\left(100 \mathrm{MHz}, \mathrm{CDCl}_{3}\right) \delta 154.3,143.4,143.3,129.2,128.1,125.8,121.6,118.4$, 115.7, 82.0, 28.1, 19.9; FT-IR $\left(\mathrm{CHCl}_{3}\right)$ 3357, 2976, 2930, 2592, 1920, 1717, 1701, 1598, 1475 $\mathrm{cm}^{-1}$; HRMS Calcd for $\mathrm{C}_{32} \mathrm{H}_{42} \mathrm{~N}_{4} \mathrm{NaO}_{4}\left(\mathrm{M}+\mathrm{Na}^{+}\right)$: 569.3104, found 569.3113 .

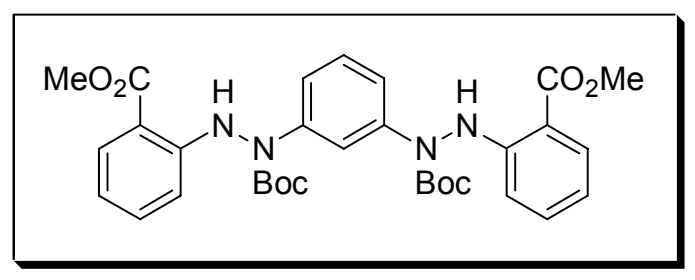

Di-tert-butyl1,1'-(1,3-phenylene)bis(2-(2-(methoxycarbonyl)phenyl)hydrazinecarboxy late) (9d)

${ }^{1} \mathrm{H}$ NMR $\left(400 \mathrm{MHz}, \mathrm{CDCl}_{3}\right) \delta 9.62(\mathrm{~s}, 2 \mathrm{H}), 7.95-7.91(\mathrm{~m}, 3 \mathrm{H}), 7.38-7.33(\mathrm{~m}, 4 \mathrm{H}), 7.27(\mathrm{t}, J=$ $3.2 \mathrm{~Hz}, 1 \mathrm{H}), 6.89(\mathrm{~d}, \mathrm{~J}=8.4 \mathrm{~Hz}, 2 \mathrm{H}), 6.84-6.80(\mathrm{~m}, 2 \mathrm{H}), 3.90(\mathrm{~s}, 6 \mathrm{H}), 1.34(\mathrm{~s}, 18 \mathrm{H}) ;{ }^{13} \mathrm{C} \mathrm{NMR}$ $\left(100 \mathrm{MHz}, \mathrm{CDCl}_{3}\right) \delta 186.2,153.4,151.4,143.0,134.5,131.2,128.5,118.6,117.5,114.9,112.7$, 112.0, 82.2, 51.8, 27.9; FT-IR $\left(\mathrm{CHCl}_{3}\right) 3327,2977,1718,1698,1589,1500,1325,1257 \mathrm{~cm}^{-1}$; 
HRMS Calcd for $\mathrm{C}_{32} \mathrm{H}_{38} \mathrm{~N}_{4} \mathrm{NaO}_{8}\left(\mathrm{M}+\mathrm{Na}^{+}\right): 629.2587$, found 629.2572 .

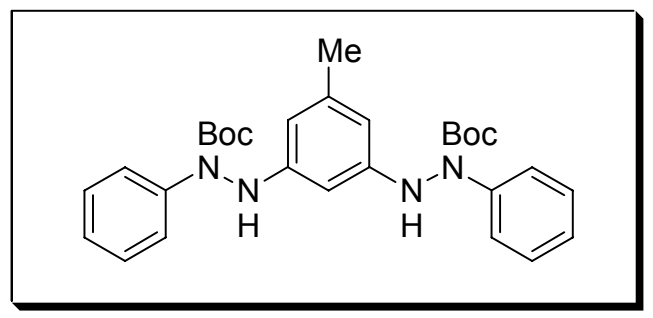

N'-[3-(tert-Butoxycarbonyl-N'-phenyl-hydrazino)-5-methyl-phenyl]-N-phenyl-

hydrazinecarboxylic aicd tert-butyl ester (9e)

${ }^{1} \mathrm{H}$ NMR $\left(400 \mathrm{MHz}, \mathrm{CDCl}_{3}\right) \delta 7.51(\mathrm{~d}, \mathrm{~J}=7.6 \mathrm{~Hz}, 4 \mathrm{H}), 7.30-7.26(\mathrm{~m}, 4 \mathrm{H}), 7.11-7.07(\mathrm{~m}, 2 \mathrm{H})$, $6.31(\mathrm{~s}, 2 \mathrm{H}), 6.18(\mathrm{~d}, \mathrm{~J}=2.0 \mathrm{~Hz}, 2 \mathrm{H}), 6.07(\mathrm{t}, \mathrm{J}=1.6 \mathrm{~Hz}, 1 \mathrm{H}), 2.19(\mathrm{~s}, 3 \mathrm{H}), 1.36(\mathrm{~s}, 18 \mathrm{H}) ;{ }^{13} \mathrm{C}$ NMR $\left(100 \mathrm{MHz}, \mathrm{CDCl}_{3}\right) \delta 153.9,149.3,142.7,140.1,128.4,124.4,121.7,106.7,95.0,82.1$, 28.0, 21.7; FT-IR $\left(\mathrm{CHCl}_{3}\right) 3340,2977,2929,2256,1715,1608,1495,1368,1338 \mathrm{~cm}^{-1}$; HRMS Calcd for $\mathrm{C}_{29} \mathrm{H}_{36} \mathrm{~N}_{4} \mathrm{NaO}_{4}\left(\mathrm{M}+\mathrm{Na}^{+}\right): 527.2634$, found 527.2622 .

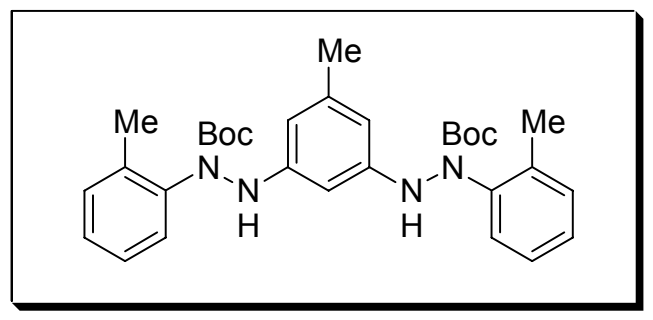

$N^{\prime}$-[3-(tert-Butoxycarbonyl-N'-phenyl-hydrazino)-5-methyl-phenyl]-N-o-tolyl-

hydrazinecarboxylic aicd tert-butyl ester (9f)

${ }^{1} \mathrm{H}$ NMR $\left(400 \mathrm{MHz}, \mathrm{CDCl}_{3}\right) \delta 7.36-7.34(\mathrm{~m}, 2 \mathrm{H}), 7.21-7.11(\mathrm{~m}, 6 \mathrm{H}), 6.38(\mathrm{~s}, 1 \mathrm{H}), 6.32$ (bs, $2 \mathrm{H}), 6.27(\mathrm{~s}, 2 \mathrm{H}), 2.31(\mathrm{~s}, 6 \mathrm{H}), 2.22(\mathrm{~s}, 3 \mathrm{H}), 1.36(\mathrm{~s}, 18 \mathrm{H}) ;{ }^{13} \mathrm{C} \mathrm{NMR}\left(100 \mathrm{MHz}, \mathrm{CDCl}_{3}\right) \delta 155.0$, $149.1,141.5,139.7,134.3,130.8,127.0,126.6,126.3,107.4,96.0,81.5,28.0,21.7,18.4$; FTIR $\left(\mathrm{CHCl}_{3}\right)$ 3336, 2976, 2928, 2583, 2135, 1715, 1702, 1520, 1507, 1456, 1367, $1158 \mathrm{~cm}^{-1}$; HRMS Calcd for $\mathrm{C}_{31} \mathrm{H}_{40} \mathrm{~N}_{4} \mathrm{NaO}_{4}\left(\mathrm{M}+\mathrm{Na}^{+}\right): 555.2947$, found 555.2944.

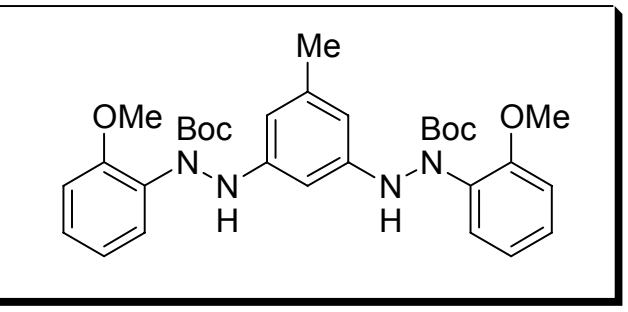


$N^{\prime}-\left\{3-\left[N^{\prime}-t e r t-B u t o x y c a r b o n y l-N\right.\right.$ '-(2-methoxy-phenyl)-hydrazino]-5-methyl-phenyl\}-N-(2methoxy-phenyl)-hydrazinecarboxylic aicd tert-butyl ester (9g)

${ }^{1} \mathrm{H} \mathrm{NMR}\left(400 \mathrm{MHz}, \mathrm{CDCl}_{3}\right) \delta 7.38\left(\mathrm{dd}, \mathrm{J}_{1}=0.8 \mathrm{~Hz}, \mathrm{~J}_{2}=1.6 \mathrm{~Hz}, 2 \mathrm{H}\right), 7.24-7.19(\mathrm{~m}, 2 \mathrm{H}), 6.91-$ $6.87(\mathrm{~m}, 4 \mathrm{H}), 6.43(\mathrm{~s}, 3 \mathrm{H}), 6.22(\mathrm{~s}, 2 \mathrm{H}), 3.87(\mathrm{~s}, 6 \mathrm{H}), 2.20(\mathrm{~s}, 3 \mathrm{H}), 1.38(\mathrm{~s}, 18 \mathrm{H}) ;{ }^{13} \mathrm{C}$ NMR $(100$ $\left.\mathrm{MHz}, \mathrm{CDCl}_{3}\right) \delta 156.1,154.4,148.8,139.5,128.7,128.44,128.40,120.4,111.5,106.7,95.5$, 81.1, 55.5, 28.1, 21.8; FT-IR $\left(\mathrm{CHCl}_{3}\right)$ 3350, 2976, 2929, 2594, 1730, 1718, 1526, 1505, 1458, $1158 \mathrm{~cm}^{-1}$; HRMS Calcd for $\mathrm{C}_{31} \mathrm{H}_{40} \mathrm{~N}_{4} \mathrm{NaO}_{6}\left(\mathrm{M}+\mathrm{Na}^{+}\right): 587.2846$, found 587.2855.

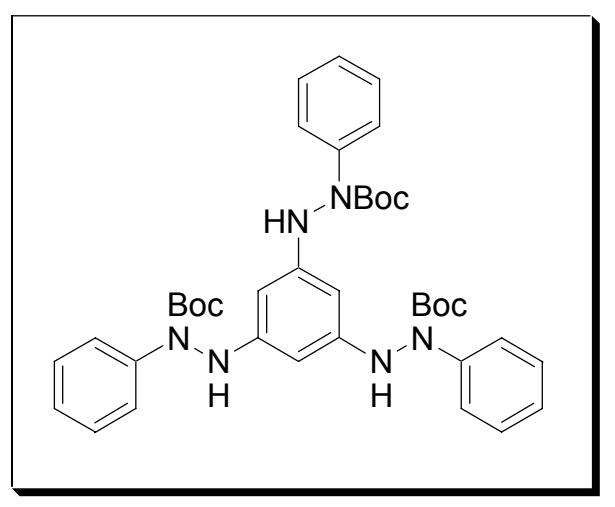

$N^{\prime}$-[3,5-Bis-( $N$ '-tert-butoxycarbonyl- $N$ '-phenyl-hydrazino)-phenyl]- $N$-phenyl-

hydrazinecarboxylic acid tert-butyl ester (11a)

${ }^{1} \mathrm{H} \mathrm{NMR}\left(400 \mathrm{MHz}, \mathrm{CDCl}_{3}\right) \delta 7.43(\mathrm{~d}, \mathrm{~J}=8.0 \mathrm{~Hz}, 6 \mathrm{H}), 7.21(\mathrm{t}, \mathrm{J}=8.8 \mathrm{~Hz}, 6 \mathrm{H}), 7.03(\mathrm{t}, J=7.2 \mathrm{~Hz}$, $3 \mathrm{H}), 6.43$ (bs, 3H), 5.81 (s, 3H), $1.32(\mathrm{~s}, 27 \mathrm{H}) ;{ }^{13} \mathrm{C} \mathrm{NMR}\left(100 \mathrm{MHz}, \mathrm{CDCl}_{3}\right) \delta 153.6,150.1$, 142.5, 128.1, 124.2, 121.8, 90.7, 82.0, 28.1; FT-IR $\left(\mathrm{CHCl}_{3}\right)$ 3344, 2978, 2931, 1711, 1616, 1495 , 1339, $1156 \mathrm{~cm}^{-1}$; HRMS Calcd for $\mathrm{C}_{39} \mathrm{H}_{49} \mathrm{~N}_{6} \mathrm{O}_{6}\left(\mathrm{M}^{+}\right): 697.3714$, found 697.3687 .

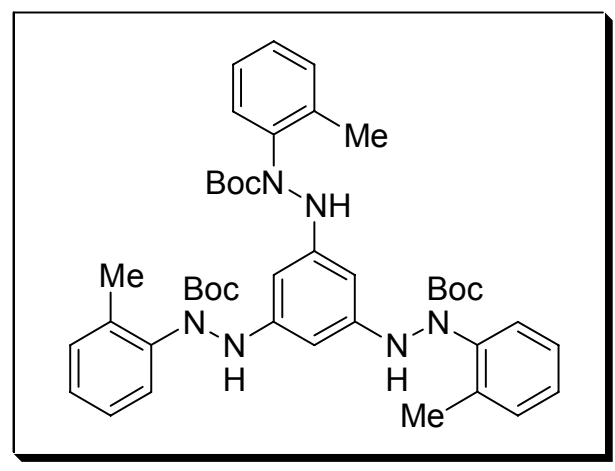

$N^{\prime}$-[3,5-Bis-(N'-tert-butoxylcarbonyl-N'-o-tolyl-hydrazino)-phenyl]-N-o-tolyl-

hydrazinecarboxylic acid tert-butyl ester (11b)

${ }^{1} \mathrm{H} \mathrm{NMR}\left(400 \mathrm{MHz}, \mathrm{CDCl}_{3}\right) \delta 7.33(\mathrm{~d}, \mathrm{~J}=7.2 \mathrm{~Hz}, 3 \mathrm{H}), 7.19-7.08(\mathrm{~m}, 9 \mathrm{H}), 6.30$ (bs, 3H), 6.08 (s, $3 \mathrm{H}), 2.29$ (s, 9H), $1.36(\mathrm{~s}, 27 \mathrm{H}) ;{ }^{13} \mathrm{C} \mathrm{NMR}\left(100 \mathrm{MHz}, \mathrm{CDCl}_{3}\right) \delta 154.8,150.1,141.4,134.3,130.8$, 
127.0, 126.7, 126.3, 92.1, 81.5, 28.0, 18.4; FT-IR $\left(\mathrm{CHCl}_{3}\right)$ 3337, 2977, 2930, 2580, 2124, 1716, 1616, 1519, 1300, $1158 \mathrm{~cm}^{-1}$; HRMS Calcd for $\mathrm{C}_{42} \mathrm{H}_{54} \mathrm{~N}_{6} \mathrm{NaO}_{6}\left(\mathrm{M}+\mathrm{Na}^{+}\right): 761.4003$, found 761.4000 .

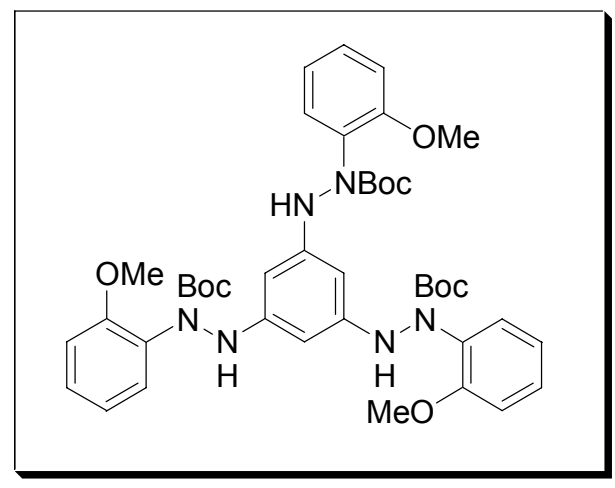

$N^{\prime}$-[3,5-Bis-( $N$ '-tert-butoxycarbonyl- $N$ '-(2-methoxy-phenyl)-hydrazino)-phenyl]- $N$-(2methoxy-phenyl)-hydrazinecarboxylic acid tert-butyl ester (11c)

${ }^{1} \mathrm{H}$ NMR $\left(400 \mathrm{MHz}, \mathrm{CDCl}_{3}\right) \delta 7.35$ - $7.32(\mathrm{~m}, 3 \mathrm{H}), 7.19-7.15(\mathrm{~m}, 3 \mathrm{H}), 6.87$ - $6.81(\mathrm{~m}, 6 \mathrm{H}), 6.36$ (bs, 3H), $6.03(\mathrm{~s}, 3 \mathrm{H}), 3.84(\mathrm{~s} 9 \mathrm{H}), 1.35(\mathrm{~s}, 27 \mathrm{H}) ;{ }^{13} \mathrm{C} \mathrm{NMR}\left(100 \mathrm{MHz}, \mathrm{CDCl}_{3}\right) \delta 155.7,154.1$, 149.5, 131.4, 128.7, 129.2, 120.3, 111.3, 91.2, 81.0, 55.5, 28.2; FT-IR $\left(\mathrm{CHCl}_{3}\right)$ 3340, 2977, 1715, 1613, 1500, 1340, $1159 \mathrm{~cm}^{-1}$; HRMS Calcd for $\mathrm{C}_{42} \mathrm{H}_{55} \mathrm{~N}_{6} \mathrm{O}_{9}\left(\mathrm{M}+\mathrm{Na}^{+}\right)$: 787.4031, found 787.4033.

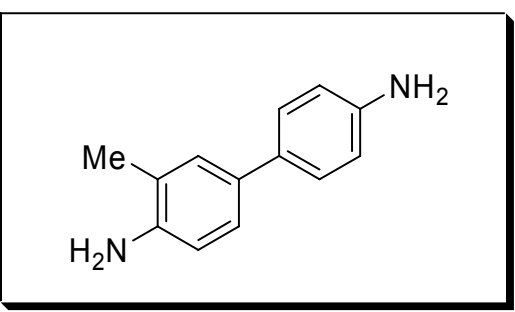

3-Methylbiphenyl-4,4'-diamine (7b)

${ }^{1} \mathrm{H}$ NMR $\left(400 \mathrm{MHz}, \mathrm{CDCl}_{3}\right) 7.35-7.33(\mathrm{~d}, \mathrm{~J}=8.0 \mathrm{~Hz}, 2 \mathrm{H}), 7.24-7.20(\mathrm{~m}, 2 \mathrm{H}), 6.74-6.69(\mathrm{~m}$, 3H), 3.64 (bs, 4H), 2.21 (s, 3H). Eggertsen, F. T.; Weiss, F. T. Anal. Chem. 1956, 28, 1008. 


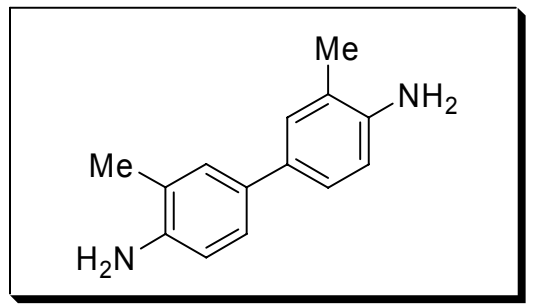

3,3'-Dimethylbiphenyl-4,4'-diamine (7c)

${ }^{1} \mathrm{H}$ NMR $\left(400 \mathrm{MHz}, \mathrm{CDCl}_{3}\right) 7.24-7.20(\mathrm{~m}, 4 \mathrm{H}), 6.70-6.68(\mathrm{~d}, \mathrm{~J}=8.0 \mathrm{~Hz}, 2 \mathrm{H}), 3.56(\mathrm{bs}, 4 \mathrm{H})$, 2.20 (s, 6H); Gupta, P. N.; Raina, A. J. Indian Chem Soc. 1989, 66, 865.

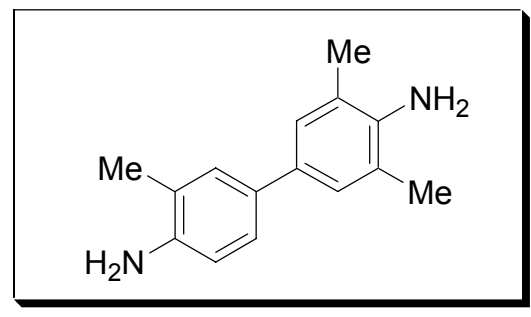

3, 5, 3'-Trimethyl-biphenyl-4, 4'-diamine (7d)

${ }^{1} \mathrm{H}$ NMR $\left(400 \mathrm{MHz}, \mathrm{CDCl}_{3}\right) \delta 7.23-7.19(\mathrm{~m}, 2 \mathrm{H}), 7.13(\mathrm{~s}, 2 \mathrm{H}), 6.66(\mathrm{~d}, \mathrm{~J}=8.0 \mathrm{~Hz}, 1 \mathrm{H}), 3.52$ (bs, $4 \mathrm{H}), 2.20(\mathrm{~s}, 6 \mathrm{H}), 2.18(\mathrm{~s}, 3 \mathrm{H}) ;{ }^{13} \mathrm{C} N M R\left(100 \mathrm{MHz}, \mathrm{CDCl}_{3}\right) \delta 143.0,141.3,132.1,131.2,128.5$, 126.3, 124.9, 122.4, 121.9, 115.2, 17.7, 17.4; FT-IR $\left(\mathrm{CHCl}_{3}\right)$ 3458, 3374, 2906, 1625, 1483 , $1267 \mathrm{~cm}^{-1}$; HRMS Calcd for $\mathrm{C}_{15} \mathrm{H}_{18} \mathrm{~N}_{2}\left(\mathrm{M}^{+}\right): 226.1470$, found 226.1474 .

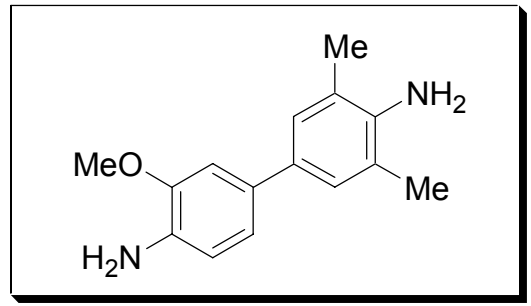

3'-Methoxy-3, 5-dimethyl-biphenyl-4, 4'-diamine (7e)

${ }^{1} \mathrm{H}$ NMR (400 MHz, $\left.\mathrm{CDCl}_{3}\right) \delta 7.13(\mathrm{~s}, 2 \mathrm{H}), 6.98-6.96(\mathrm{~m}, 2 \mathrm{H}), 6.72(\mathrm{~d}, \mathrm{~J}=8.4 \mathrm{~Hz}, 1 \mathrm{H}), 3.89$ (s, 3H), 3.66 (bs, 4H), 2.22 (s, 6H); ${ }^{13} \mathrm{C}$ NMR $\left(100 \mathrm{MHz}, \mathrm{CDCl}_{3}\right) \delta$ 147.4, 141.4, 134.5, 132.4, 131.5, 126.4, 121.9, 119.0, 115.1, 109.0, 55.4, 17.7; FT-IR $\left(\mathrm{CHCl}_{3}\right)$ 3466, 3364, 3052, 2982, 1622 , 1467, $1266 \mathrm{~cm}^{-1}$; HRMS Calcd for $\mathrm{C}_{15} \mathrm{H}_{18} \mathrm{~N}_{2} \mathrm{O}\left(\mathrm{M}^{+}\right): 242.1419$, found 242.1424 . 


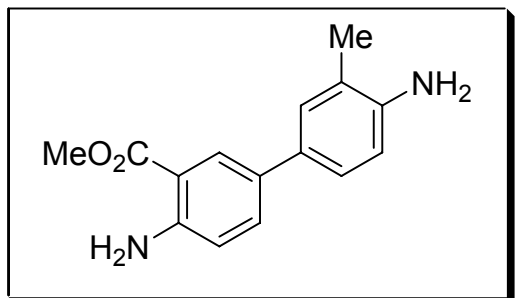

4,4'-Diamino-3'-methyl-biphenyl-3-carboxylic acid methyl ester (7f)

${ }^{1} \mathrm{H} \mathrm{NMR}\left(400 \mathrm{MHz}, \mathrm{CDCl}_{3}\right) \delta 8.04-8.03(\mathrm{~d}, \mathrm{~J}=2.2 \mathrm{~Hz}, 1 \mathrm{H}), 7.49-7.46\left(\mathrm{dd}, \mathrm{J}_{1}=8.4 \mathrm{~Hz}, \mathrm{~J}_{2}=2.2\right.$ $\mathrm{Hz}, 1 \mathrm{H}), 7.24-7.21(\mathrm{~m}, 2 \mathrm{H}), 6.72-6.70(\mathrm{~d}, \mathrm{~J}=8.0 \mathrm{~Hz}, 1 \mathrm{H}), 6.70-6.68(\mathrm{~d}, \mathrm{~J}=8.4 \mathrm{~Hz}, 1 \mathrm{H}), 3.89$ (s, 3H), $2.22(\mathrm{~s}, 3 \mathrm{H}) ;{ }^{13} \mathrm{C}$ NMR $\left(100 \mathrm{MHz}, \mathrm{CDCl}_{3}\right) \delta 169.4,149.6,144.1,133.1,131.7,130.4$, 129.2, 129.0, 125.5, 123.3, 117.8, 116.0, 111.6, 52.2, 18.2; FT-IR $\left(\mathrm{CHCl}_{3}\right) 3476,3370,2949$, 1689, 1624, 1586, 1491, 1437, 1299, 1239 $\mathrm{cm}^{-1}$; HRMS Calcd for $\mathrm{C}_{15} \mathrm{H}_{16} \mathrm{~N}_{2} \mathrm{O}_{2}\left(\mathrm{M}^{+}\right): 256.1212$, found 256.1210 .

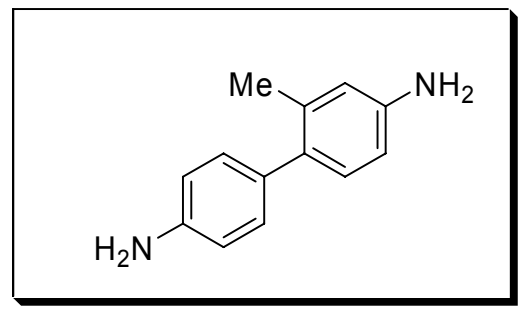

\section{2-methylbiphenyl-4,4'-diamine (14a)}

${ }^{1} \mathrm{H} \mathrm{NMR}\left(400 \mathrm{MHz}, \mathrm{CDCl}_{3}\right) 7.10-7.08(\mathrm{~d}, \mathrm{~J}=8.4 \mathrm{~Hz}, 2 \mathrm{H}), 7.02-7.00(\mathrm{~d}, \mathrm{~J}=8.0 \mathrm{~Hz}, 1 \mathrm{H}), 6.72$ $-6.70(\mathrm{~d}, \mathrm{~J}=8.4 \mathrm{~Hz}, 2 \mathrm{H}), 6.60-6.59(\mathrm{~d}, \mathrm{~J}=2.4 \mathrm{~Hz}, 1 \mathrm{H}), 6.57-6.54\left(\mathrm{dd}, \mathrm{J}_{1}=8.0 \mathrm{~Hz}, \mathrm{~J}_{2}=2.4\right.$ Hz, 1H), 3.64 (bs, 4H), 2.21 (s, 3H); Badger, G. M.; Drewer, R. J.; Lewis, G. E. Australian J. Chem. 1963, 16, 1042.

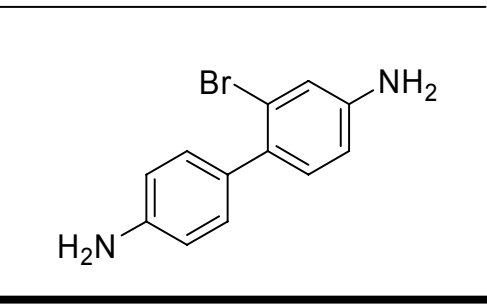

\section{2-bromobiphenyl-4,4'-diamine (14b)}

${ }^{1} \mathrm{H}$ NMR $\left(400 \mathrm{MHz}, \mathrm{CDCl}_{3}\right) 7.20-7.17(\mathrm{~d}, \mathrm{~J}=8.6 \mathrm{~Hz}, 2 \mathrm{H}), 7.09-7.07(\mathrm{~d}, \mathrm{~J}=8.2 \mathrm{~Hz}, 1 \mathrm{H}), 6.98$ $-6.97(\mathrm{~d}, J=2.8 \mathrm{~Hz}, 1 \mathrm{H}), 6.72-6.69(\mathrm{~d}, \mathrm{~J}=8.6 \mathrm{~Hz}, 2 \mathrm{H}), 6.65-6.21\left(\mathrm{dd}, \mathrm{J}_{1}=8.2 \mathrm{~Hz}, \mathrm{~J}_{2}=2.8\right.$ Hz, 1H), 3.70 (bs, 4H); Kimura, M.; Kuwano, S.; Sawaki, Y.; Fujikawa, H. J. Materials Chem. 2005, 15, 2393. 


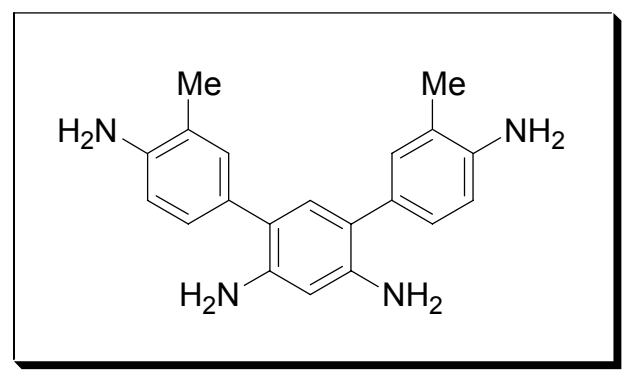

3,3"-Dimethyl-[1,1';3',1"]terphenyl-4,4',6',4"-tetraamine (10b)

${ }^{1} \mathrm{H}$ NMR $\left(400 \mathrm{MHz}, \mathrm{CDCl}_{3}\right) \delta 7.14-7.12(\mathrm{~m}, 4 \mathrm{H}), 6.90(\mathrm{~s}, 1 \mathrm{H}), 6.73-6.71$ (d, J= $\left.7.7 \mathrm{~Hz}, 2 \mathrm{H}\right)$, $6.18(\mathrm{~s}, 1 \mathrm{H}), 3.67$ (s, 8H), 2.19 (s, 6H); ${ }^{13} \mathrm{C} \mathrm{NMR}\left(100 \mathrm{MHz}, \mathrm{CDCl}_{3}\right) \delta$ 144.0, 143.8, 133.1, 132.0, 130.5, 128.4, 123.3, 120.2, 115.8, 102.8, 18.1; FT-IR $\left(\mathrm{CHCl}_{3}\right)$ 3437, 3352, 2921, 2850, 1619, 1493, 1280, $1262 \mathrm{~cm}^{-1}$; HRMS Calcd for $\mathrm{C}_{20} \mathrm{H}_{22} \mathrm{~N}_{4}\left(\mathrm{M}^{+}\right)$: 318.1844, found 318.1839.

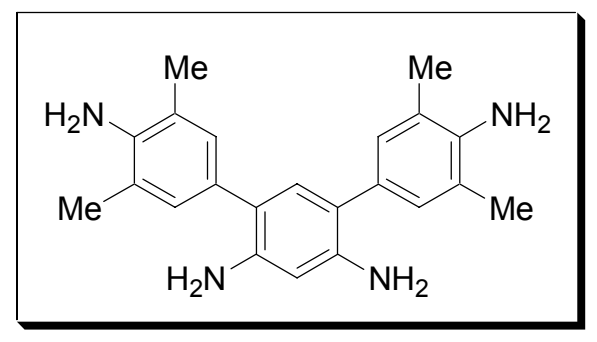

3, 5, 3", 5"-Tetramethyl-[1, 1'; 3', 1']terphenyl-4, 4', 6', 4"'-tetraamine (10c)

${ }^{1} \mathrm{H} \mathrm{NMR}\left(400 \mathrm{MHz}, \mathrm{CDCl}_{3}\right) \delta 7.05(\mathrm{~s}, 4 \mathrm{H}), 6.91$ (s, 1H), 6.19 (s, 1H), 3.72 (bs, 4H), 3.60 (bs, 4H), 2.20 (s, 12H); ${ }^{13} \mathrm{C}$ NMR $\left(100 \mathrm{MHz}, \mathrm{CDCl}_{3}\right) \delta 143.0,141.2,132.3,129.1,128.9,121.8,119.6$, 102.1, 17.8; FT-IR $\left(\mathrm{CHCl}_{3}\right) 3054,2987,2686,1621,1558,1422 \mathrm{~cm}^{-1}$; HRMS Calcd for $\mathrm{C}_{22} \mathrm{H}_{26} \mathrm{~N}_{4}$ $\left(\mathrm{M}^{+}\right)$: 346.2157, found 346.2147 .

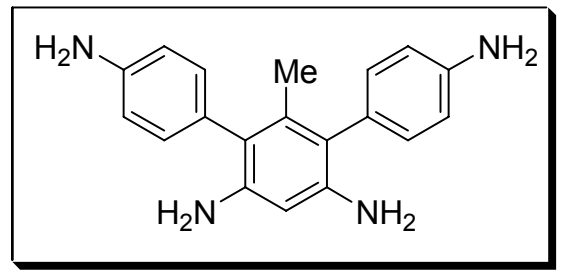

2'-Methyl-[1, 1'; 3', 1']terphenyl-4, 4', 6', 4'-tetraamine (10e)

${ }^{1} \mathrm{H}$ NMR $\left(400 \mathrm{MHz}, \mathrm{CDCl}_{3}\right) \delta 7.05(\mathrm{~d}, \mathrm{~J}=8.4 \mathrm{~Hz}, 4 \mathrm{H}), 6.79(\mathrm{~d}, \mathrm{~J}=8.4 \mathrm{~Hz}, 4 \mathrm{H}), 6.11(\mathrm{~s}, 1 \mathrm{H}), 3.56$ (bs, 8H), $1.70(\mathrm{~s}, 3 \mathrm{H}) ;{ }^{13} \mathrm{C}$ NMR $\left(100 \mathrm{MHz}, \mathrm{CDCl}_{3}\right) \delta 145.0,144.1,136.7,131.6,128.9,119.3$, 115.6, 98.9, 18.9; FT-IR $\left(\mathrm{CHCl}_{3}\right)$ 3055, 2987, 2686, 2524, 1612, 1514, 1425, $1566 \mathrm{~cm}^{-1}$; HRMS Calcd for $\mathrm{C}_{19} \mathrm{H}_{20} \mathrm{~N}_{4}\left(\mathrm{M}^{+}\right)$: 304.1688 , found 304.1673. 


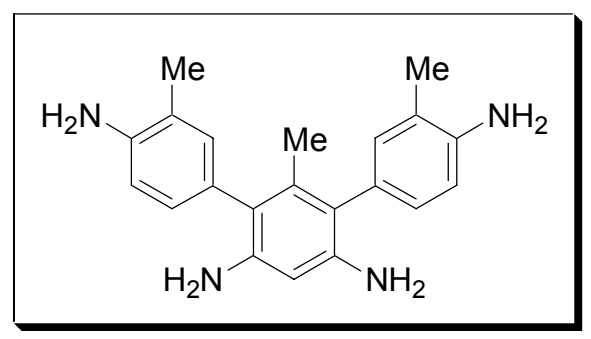

3, 2', 3"-Trimethyl-[1, 1'; 3', 1"]terphenyl-4, 4', 6', 4"-tetraamine (10f)

${ }^{1} \mathrm{H}$ NMR $\left(400 \mathrm{MHz}, \mathrm{CDCl}_{3}\right) \delta 6.95-6.91(\mathrm{~m}, 4 \mathrm{H}), 6.73(\mathrm{~d}, \mathrm{~J}=8.0 \mathrm{~Hz}, 2 \mathrm{H}), 6.10(\mathrm{~s}, 1 \mathrm{H}), 3.52$ (bs, $8 \mathrm{H}), 2.18(\mathrm{~s}, 6 \mathrm{H}), 1.70(\mathrm{~s}, 3 \mathrm{H}) ;{ }^{13} \mathrm{C}$ NMR $\left(100 \mathrm{MHz}, \mathrm{CDCl}_{3}\right) \delta 144.0,143.1,136.5,132.7,129.1$, 129.0, 122.7, 119.5, 115.4, 99.0, 18.9, 17.4; FT-IR $\left(\mathrm{CHCl}_{3}\right)$ 3054, 2986, 2927, 2686, 1610, 1460 , 1426, $1266 \mathrm{~cm}^{-1}$; HRMS Calcd for $\mathrm{C}_{21} \mathrm{H}_{24} \mathrm{~N}_{4}\left(\mathrm{M}^{+}\right)$: 332.2001, found 332.2000.

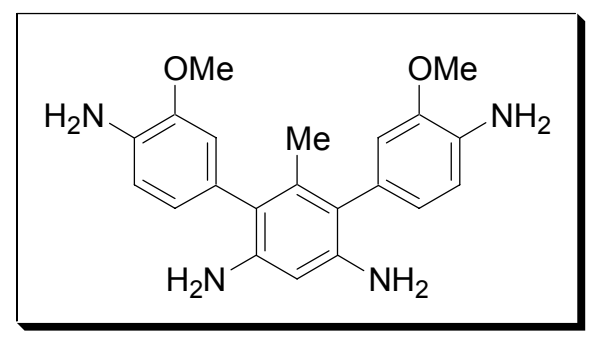

3, 3"'-Dimethoxy-2'-methyl-[1, 1'; 3', 1']terphenyl-4, 4', 6', 4"-tetraamine (10g) ${ }^{1} \mathrm{H}$ NMR $\left(400 \mathrm{MHz}, \mathrm{CDCl}_{3}\right) \delta 6.77(\mathrm{~d}, \mathrm{~J}=3.6 \mathrm{~Hz}, 2 \mathrm{H}), 6.72-6.68(\mathrm{~m}, 4 \mathrm{H}), 6.12(\mathrm{~s}, 1 \mathrm{H}), 3.83(\mathrm{~s}$, $6 \mathrm{H}), 3.66$ (bs, 8H), 1.74 (s, 3H); ${ }^{13} \mathrm{C} \mathrm{NMR}\left(100 \mathrm{MHz}, \mathrm{CDCl}_{3}\right) \delta 147.6,144.2,136.6,134.6,128.9$, 123.0, 119.5, 115.3, 112.8, 98.8, 55.5, 18.8; FT-IR $\left(\mathrm{CHCl}_{3}\right)$ 3054, 2987, 2685, 1610, 1518, 1421 , $1263 \mathrm{~cm}^{-1}$; HRMS Calcd for $\mathrm{C}_{21} \mathrm{H}_{24} \mathrm{~N}_{4} \mathrm{O}_{2}\left(\mathrm{M}^{+}\right)$: 364.1899 , found 364.1877 .

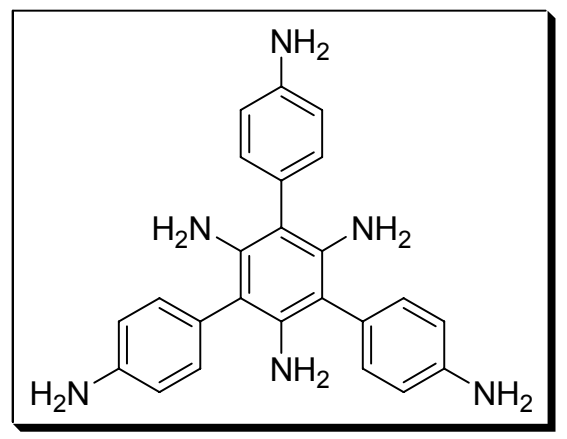

12a: ${ }^{1} \mathrm{H}$ NMR $\left(400 \mathrm{MHz}, \mathrm{CD}_{3} \mathrm{OD}\right) \delta 7.06(\mathrm{~d}, \mathrm{~J}=8.4 \mathrm{~Hz}, 6 \mathrm{H}), 6.83(\mathrm{~d}, \mathrm{~J}=8.4 \mathrm{~Hz}, 6 \mathrm{H}) ;{ }^{13} \mathrm{C}$ NMR (100 MHz, CD $\left.{ }_{3} \mathrm{OD}\right) \delta 148.5,143.1,133.3,127.1,117.9,109.1 ; \mathrm{FT}-\mathrm{IR}\left(\mathrm{CHCl}_{3}\right) 3054,2986,2931$, $1715,1605,1514,1447,1422,1266 \mathrm{~cm}^{-1}$; HRMS Calcd for $\mathrm{C}_{24} \mathrm{H}_{24} \mathrm{~N}_{6}\left(\mathrm{M}^{+}\right): 396.2062$, found 396.2061. 


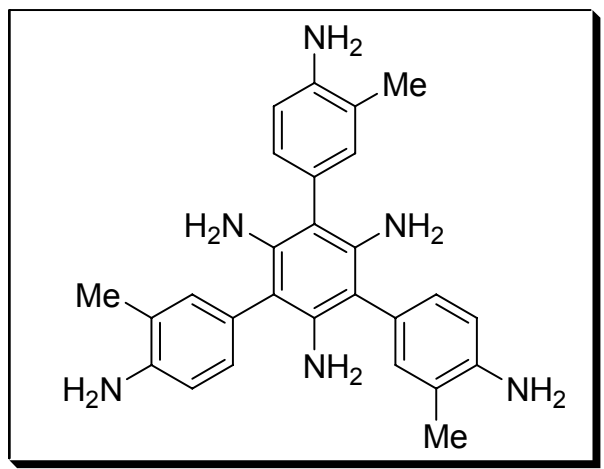

12b: ${ }^{1} \mathrm{H}$ NMR (400 MHz, $\left.\mathrm{CDCl}_{3}\right) \delta 7.13(\mathrm{~s}, 3 \mathrm{H}), 7.09\left(\mathrm{dd}, \mathrm{J}_{1}=1.6 \mathrm{~Hz}, \mathrm{~J}_{2}=2.0 \mathrm{~Hz}, 3 \mathrm{H}\right), 6.76(\mathrm{~d}, \mathrm{~J}$ $=7.6 \mathrm{~Hz}, 3 \mathrm{H}), 3.62(\mathrm{bs}, 6 \mathrm{H}), 3.31$ (bs, 6H), $2.18(\mathrm{~s}, 9 \mathrm{H}) ;{ }^{13} \mathrm{C} \mathrm{NMR}\left(100 \mathrm{MHz}, \mathrm{CDCl}_{3}\right) \delta 143.6$, 142.1, 133.4, 129.9, 126.5, 123.4, 115.9, 105.1, 17.4; FT-IR $\left(\mathrm{CHCl}_{3}\right)$ 3054, 2987, 2830, 1611 , 1517, 1422, $1266 \mathrm{~cm}^{-1}$; HRMS Calcd for $\mathrm{C}_{27} \mathrm{H}_{30} \mathrm{~N}_{6}\left(\mathrm{M}^{+}\right)$: 438.2532, found 438.2522.

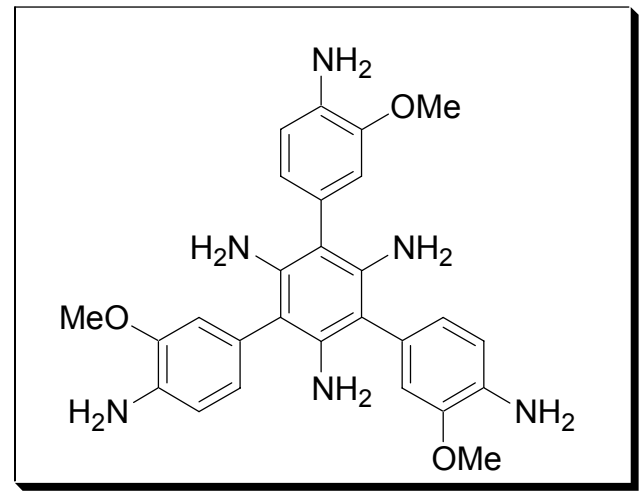

12c: ${ }^{1} \mathrm{H}$ NMR (400 MHz, $\left.\mathrm{CDCl}_{3}\right) \delta 6.88-6.80(\mathrm{~m}, 9 \mathrm{H}), 3.84$ (s, $\left.15 \mathrm{H}\right), 3.39$ (bs, $\left.6 \mathrm{H}\right) ;{ }^{13} \mathrm{C} \mathrm{NMR}$ $\left(100 \mathrm{MHz}, \mathrm{CDCl}_{3}\right) \delta 148.0,142.5,135.1,126.3,123.8,115.8,113.3,104.8,55.5 ; \mathrm{FT}-\mathrm{IR}\left(\mathrm{CHCl}_{3}\right)$ $3054,2987,2835,1733,1603,1517,1440,1422,1265 \mathrm{~cm}^{-1}$; HRMS Calcd for $\mathrm{C}_{27} \mathrm{H}_{30} \mathrm{~N}_{6} \mathrm{O}_{3}\left(\mathrm{M}^{+}\right)$: 486.2379 , found 486.2366 . 


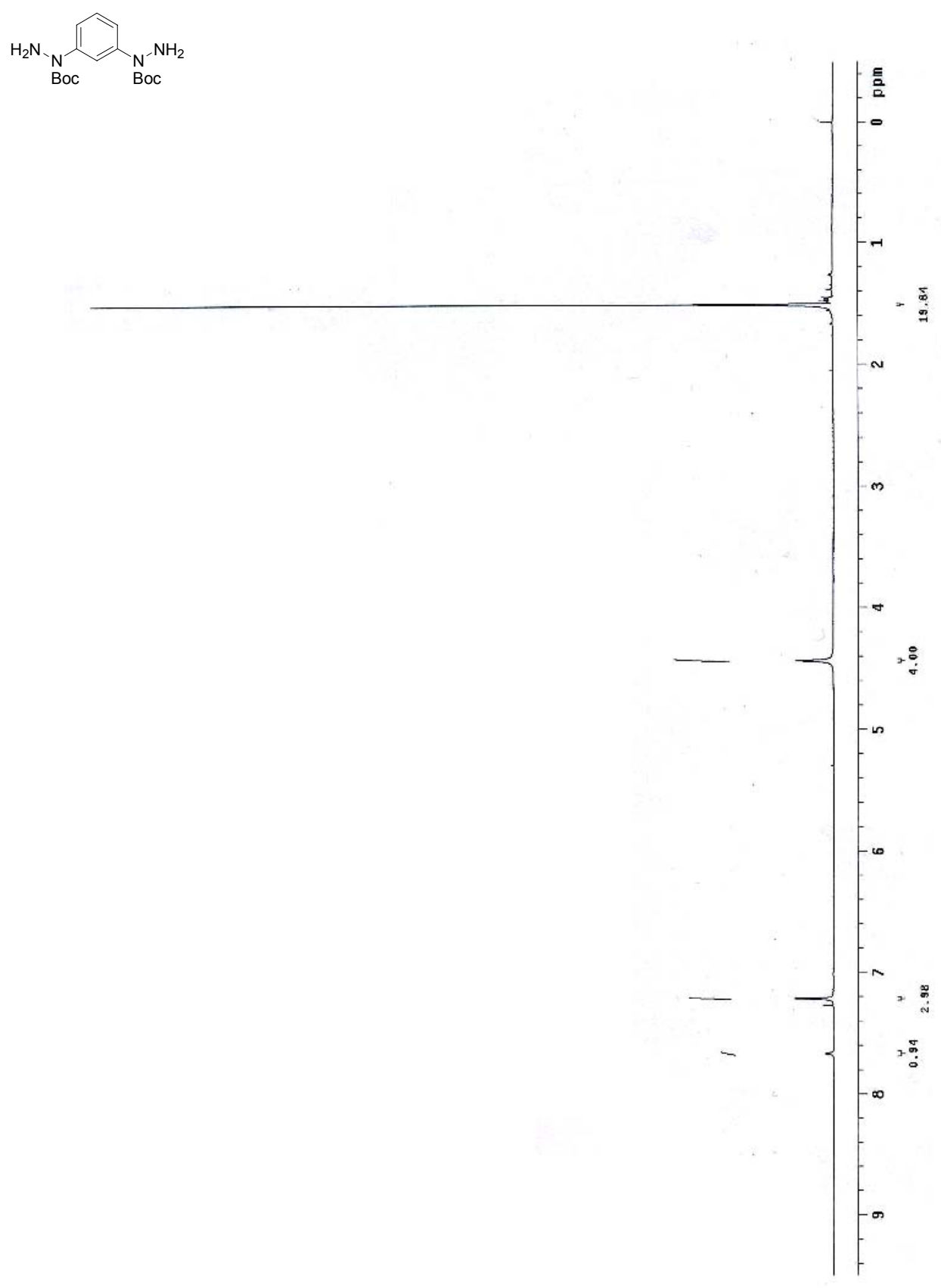



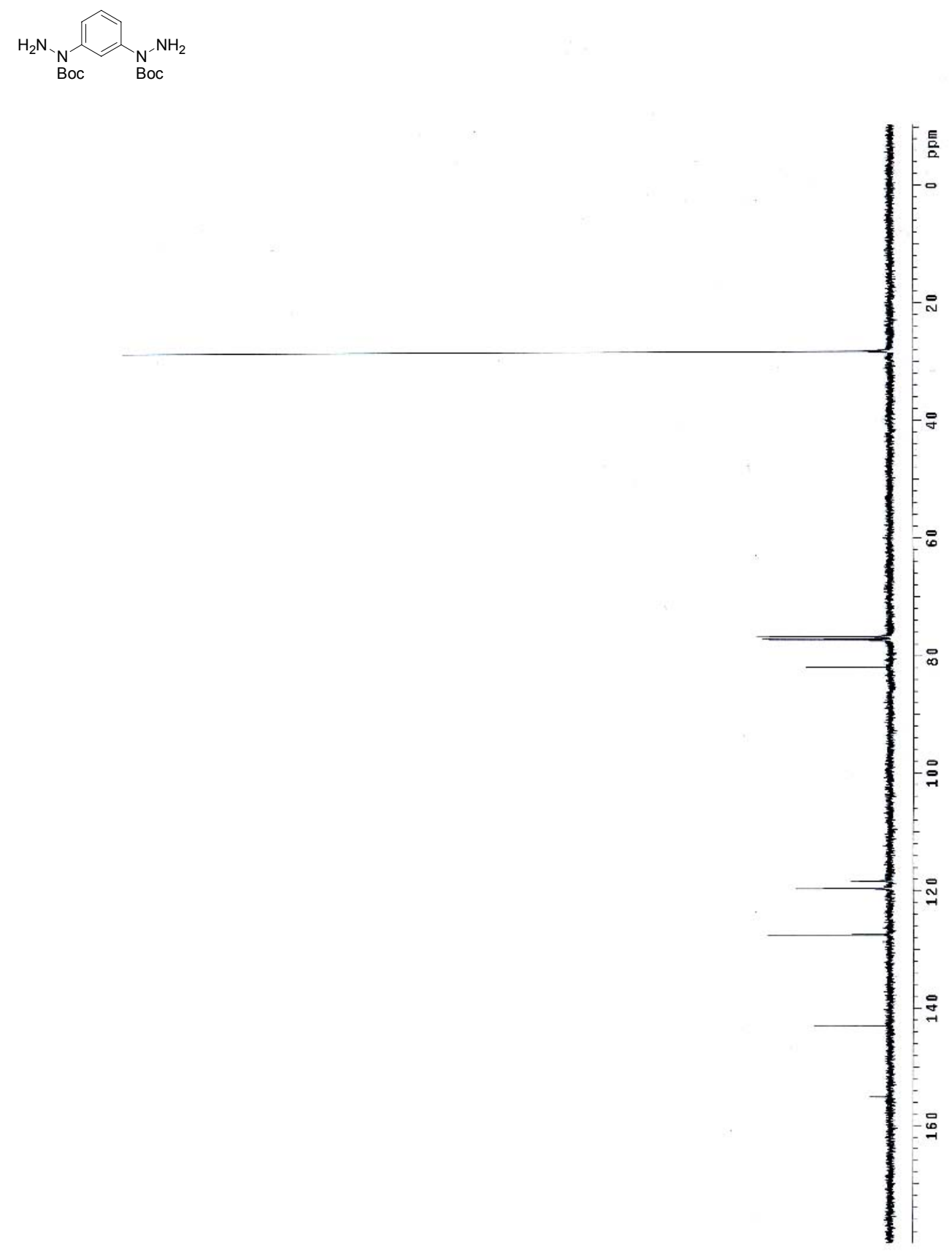

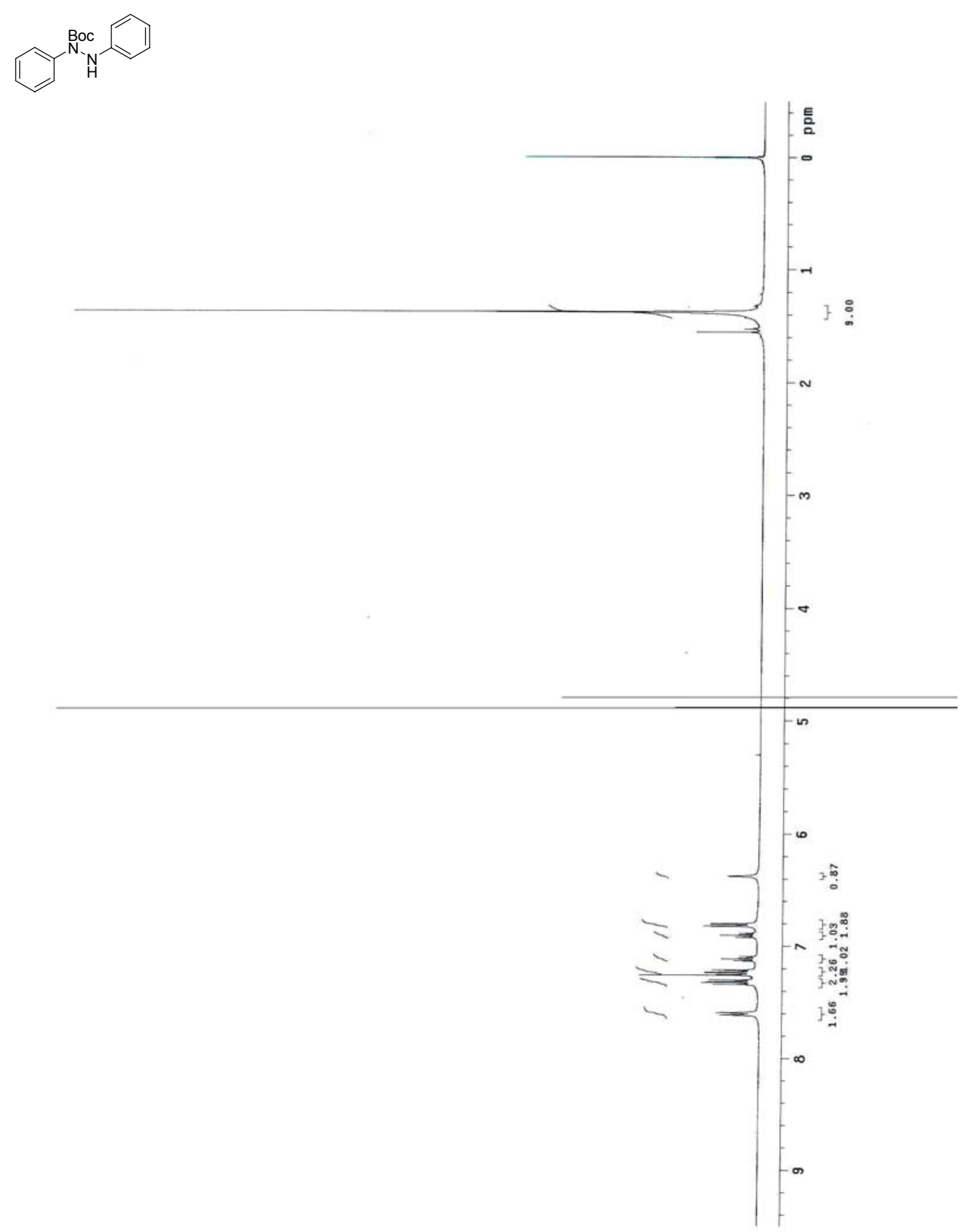

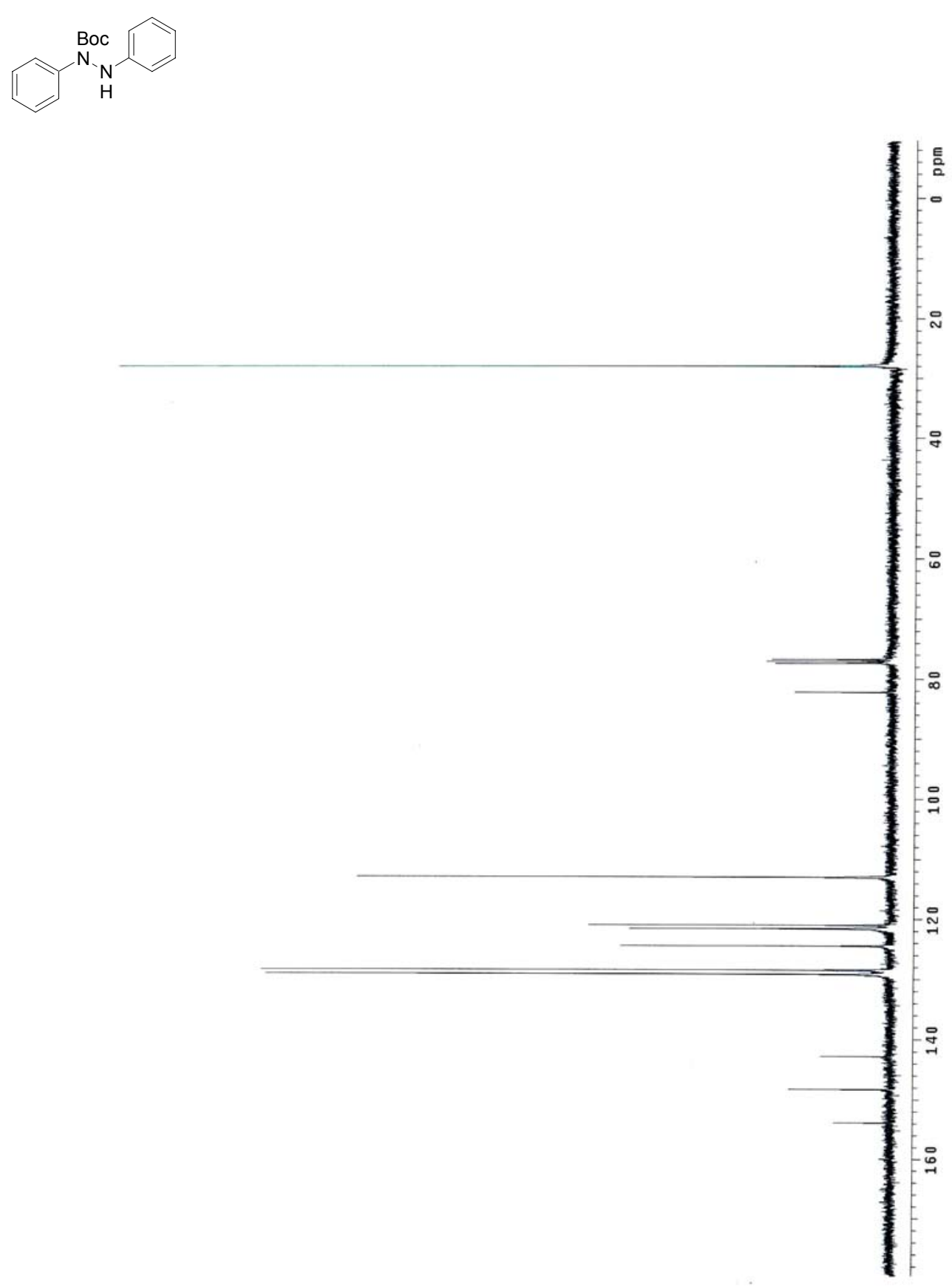

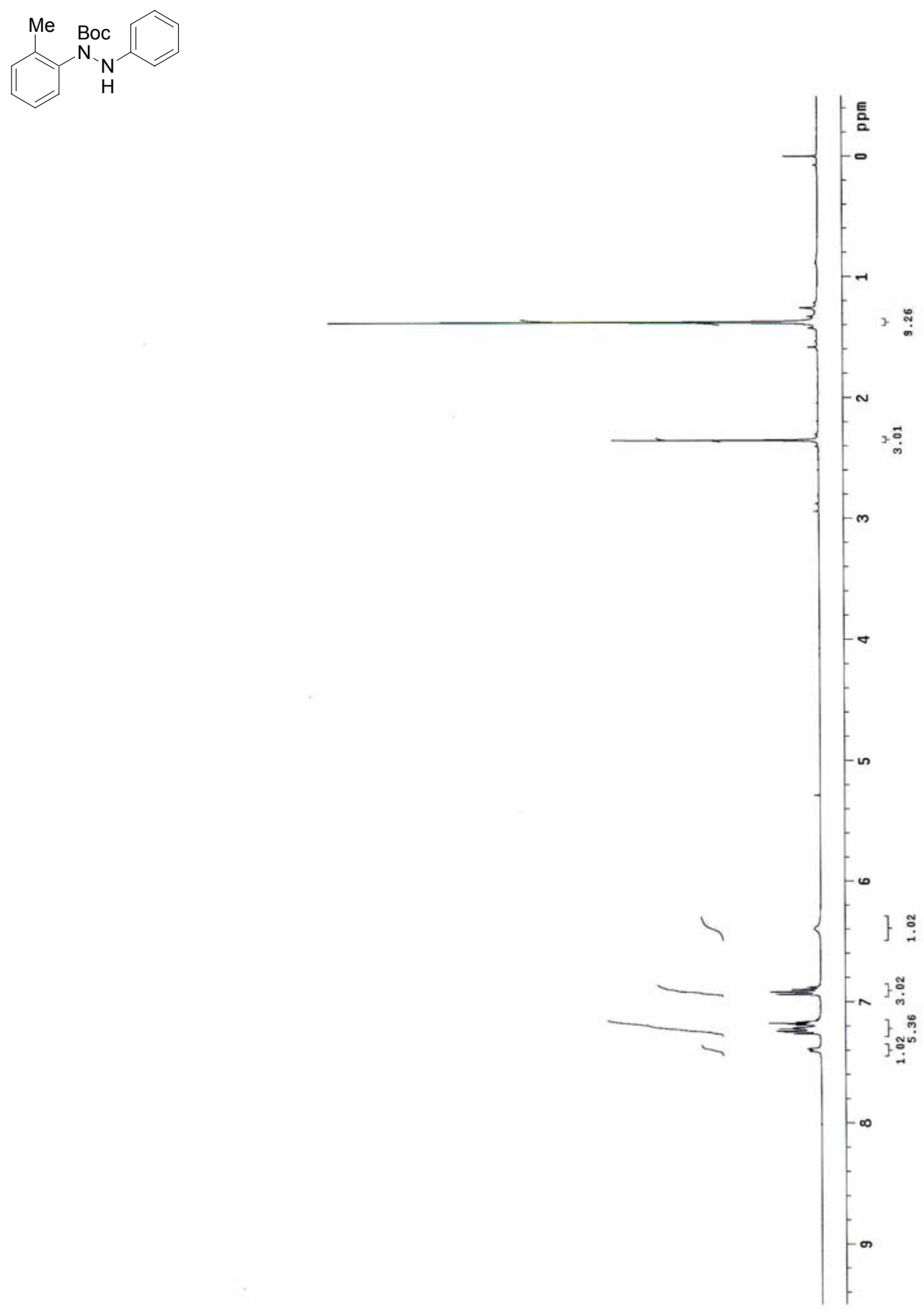


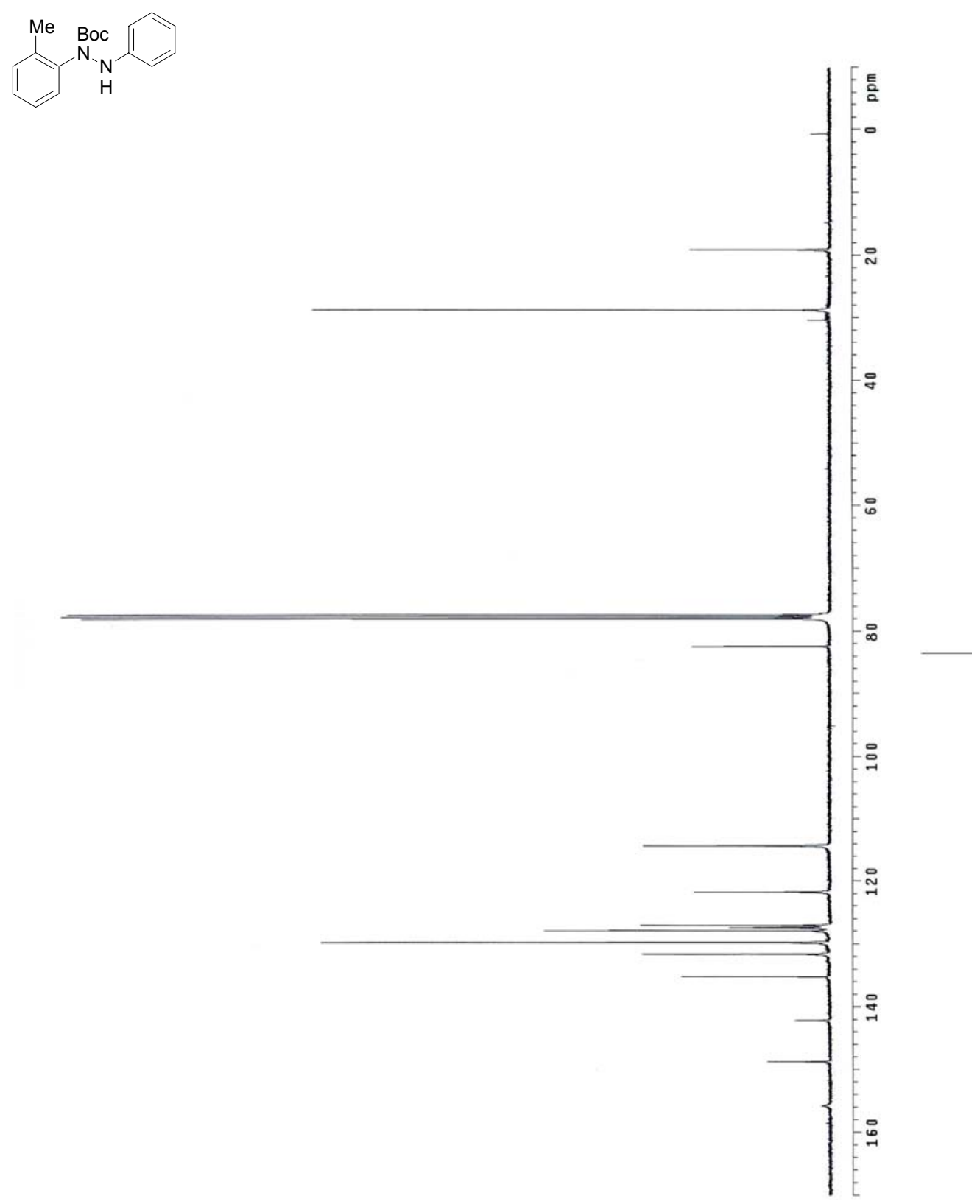




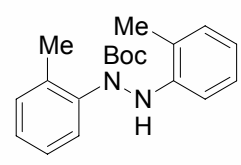

$\mathrm{H}$

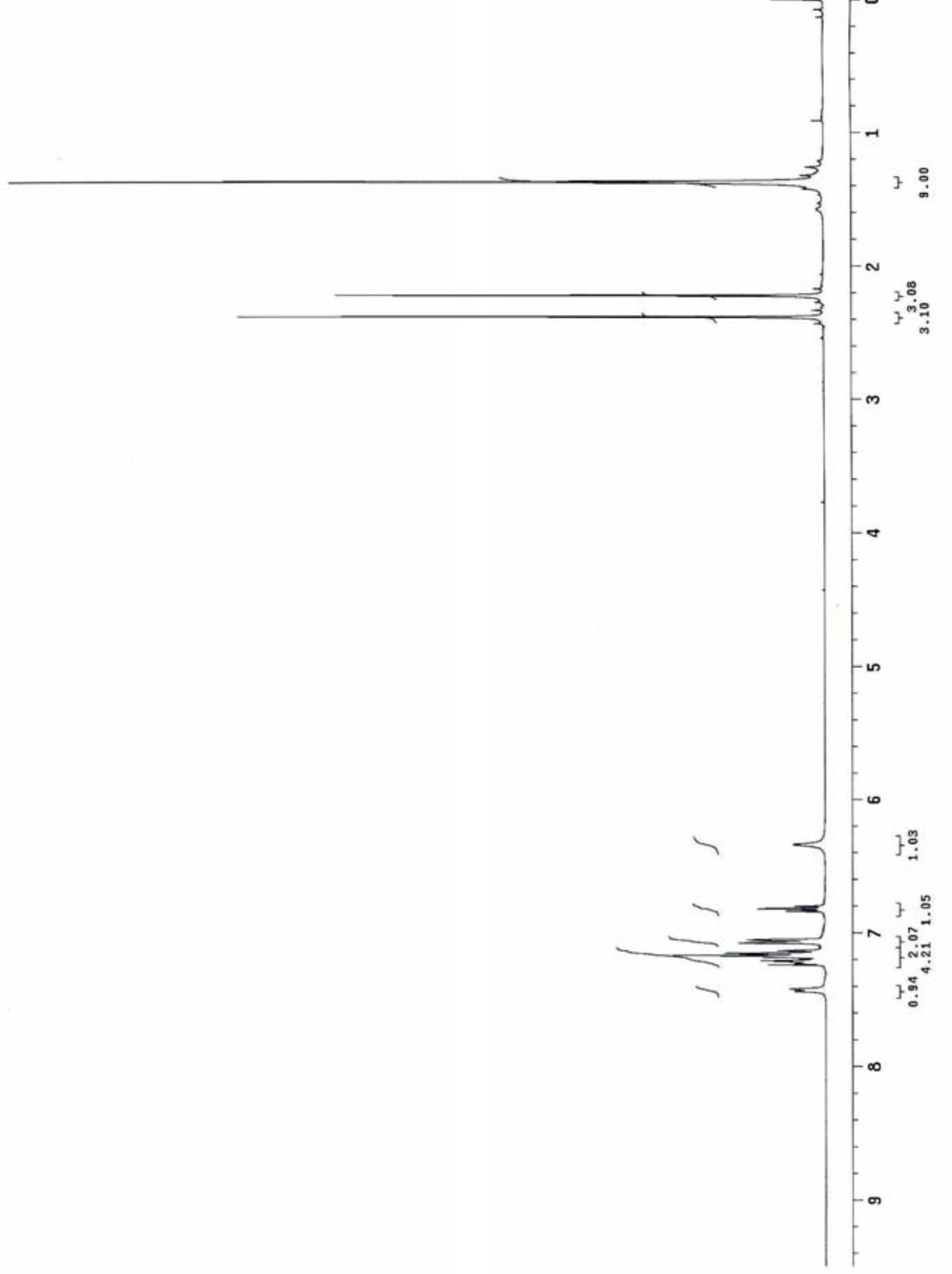



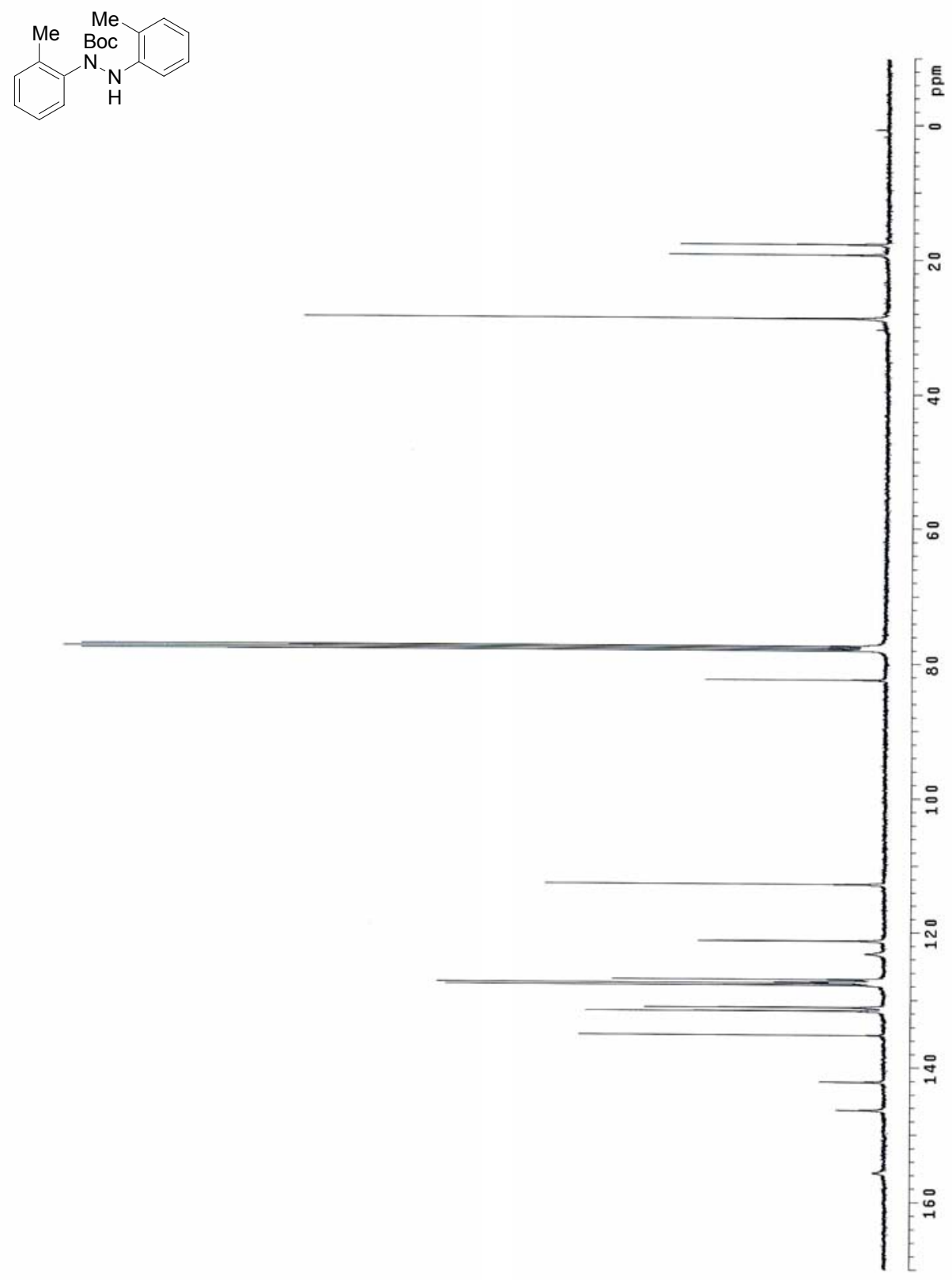

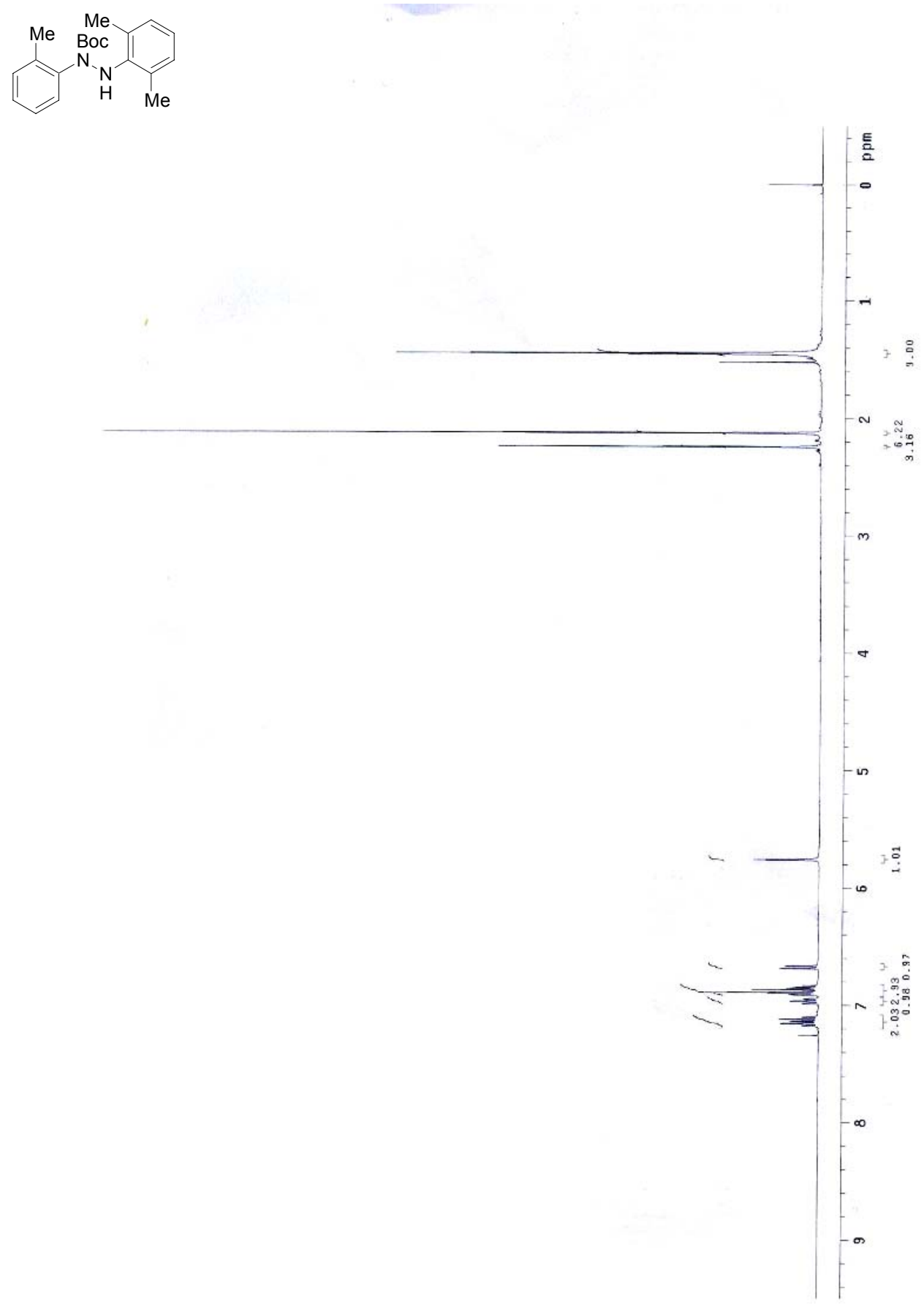

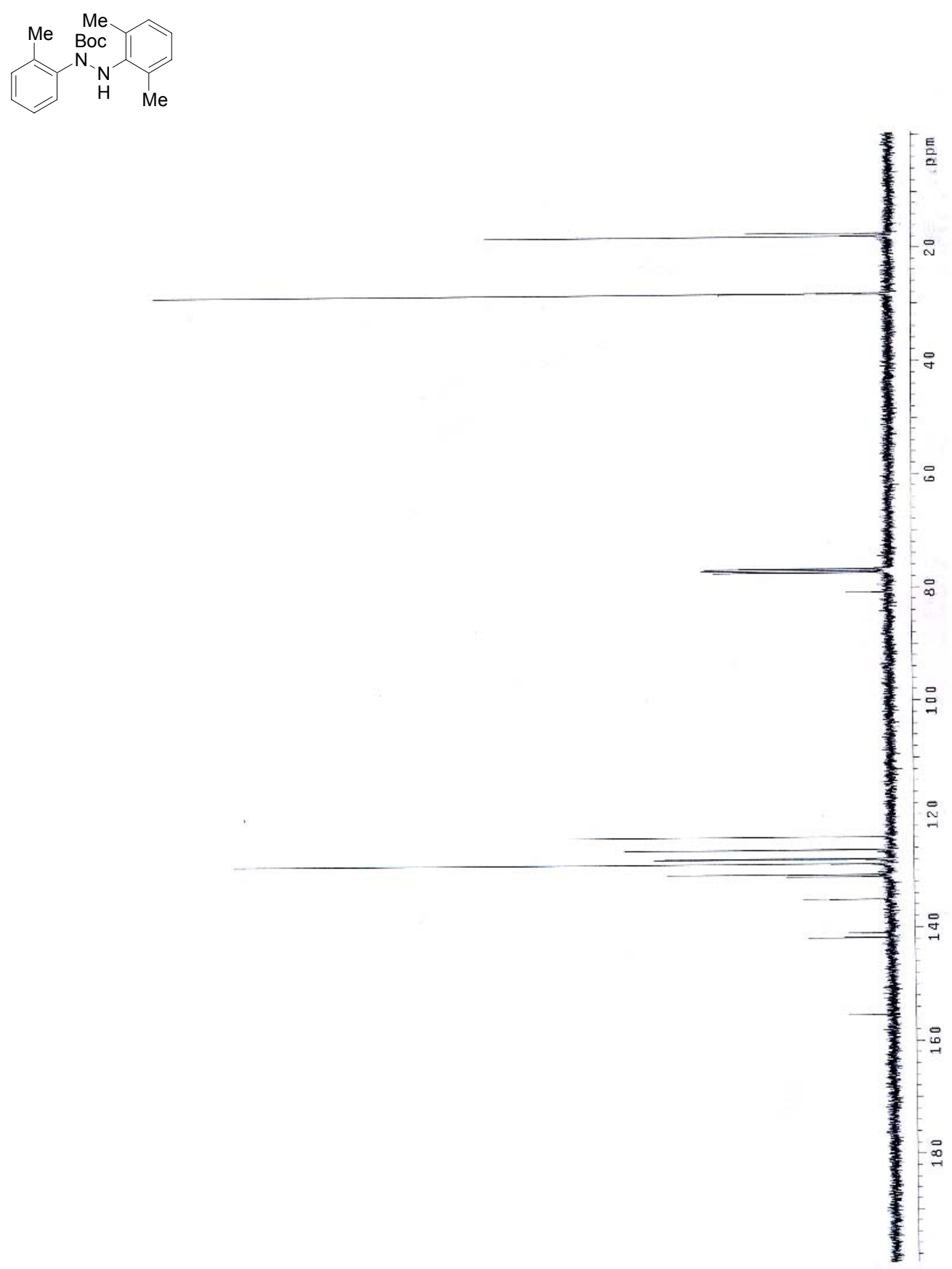

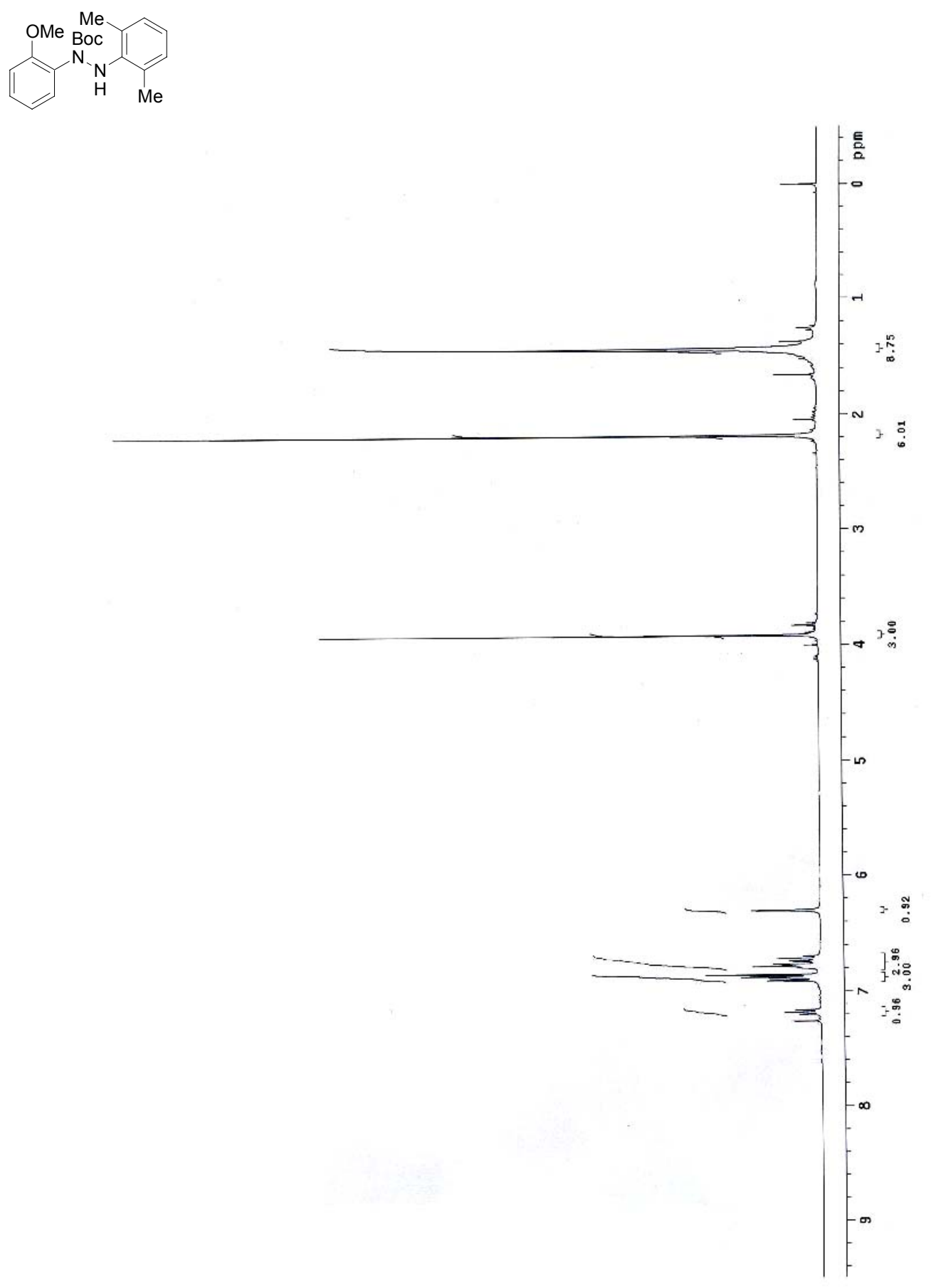

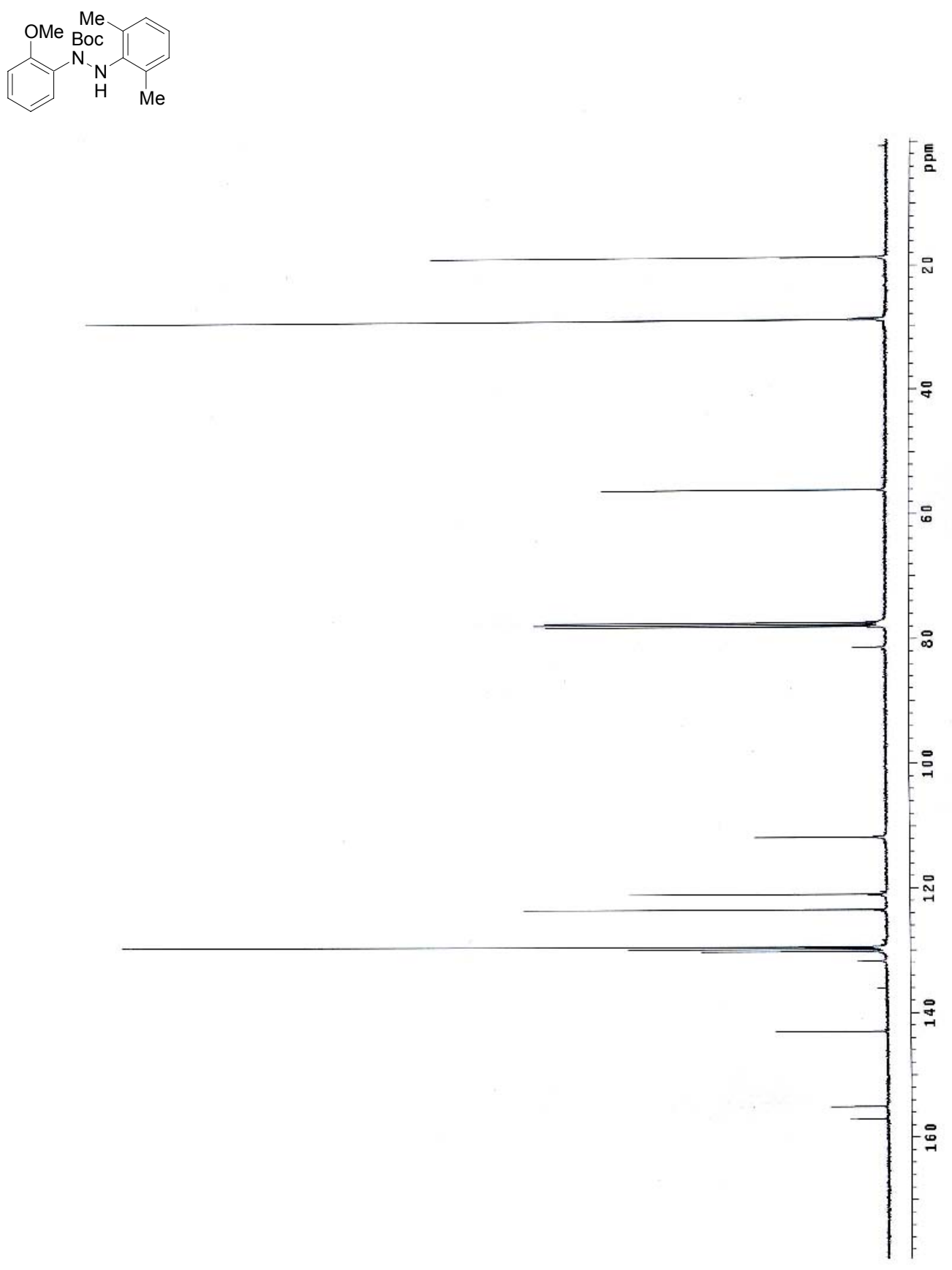


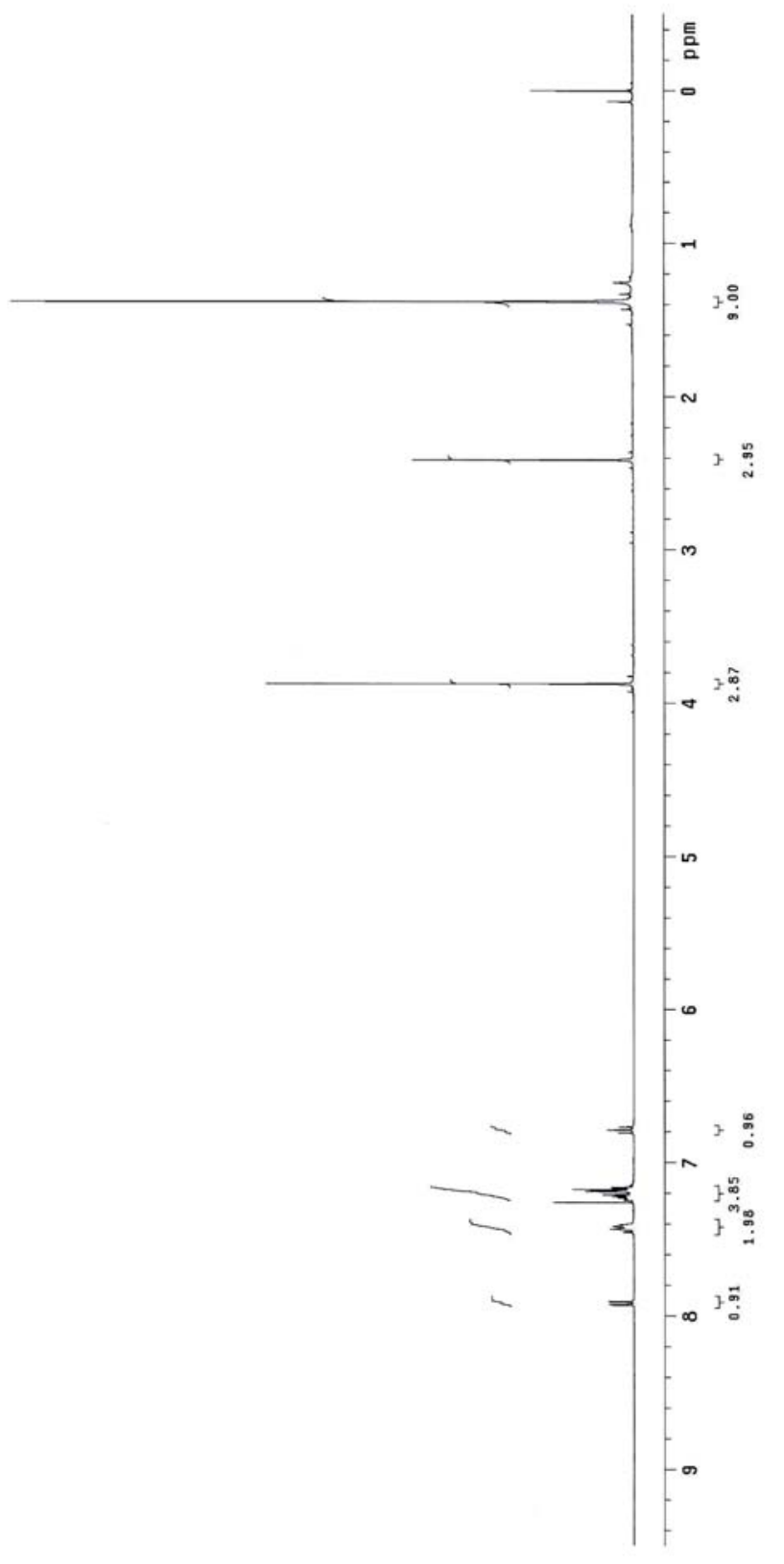



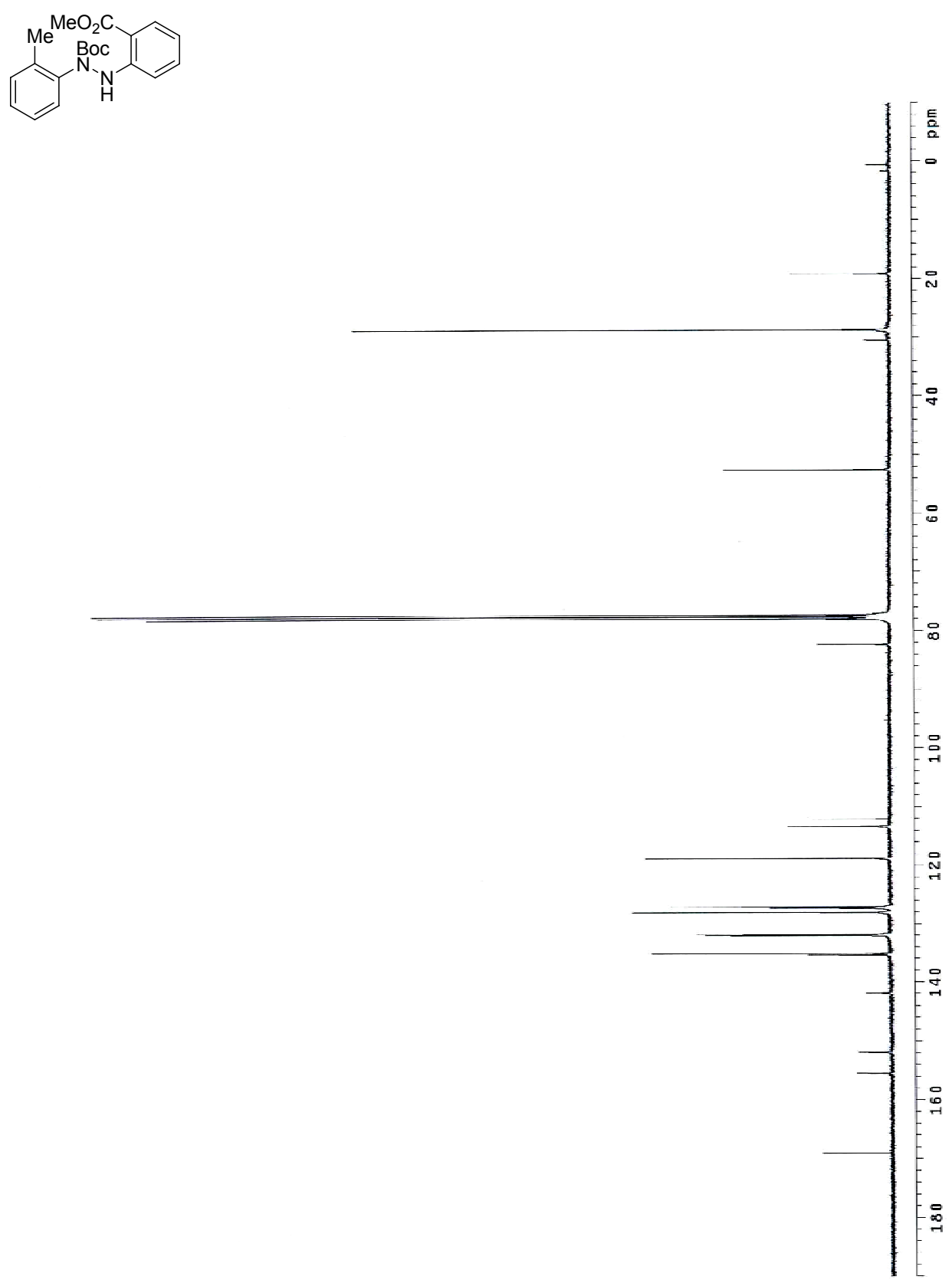

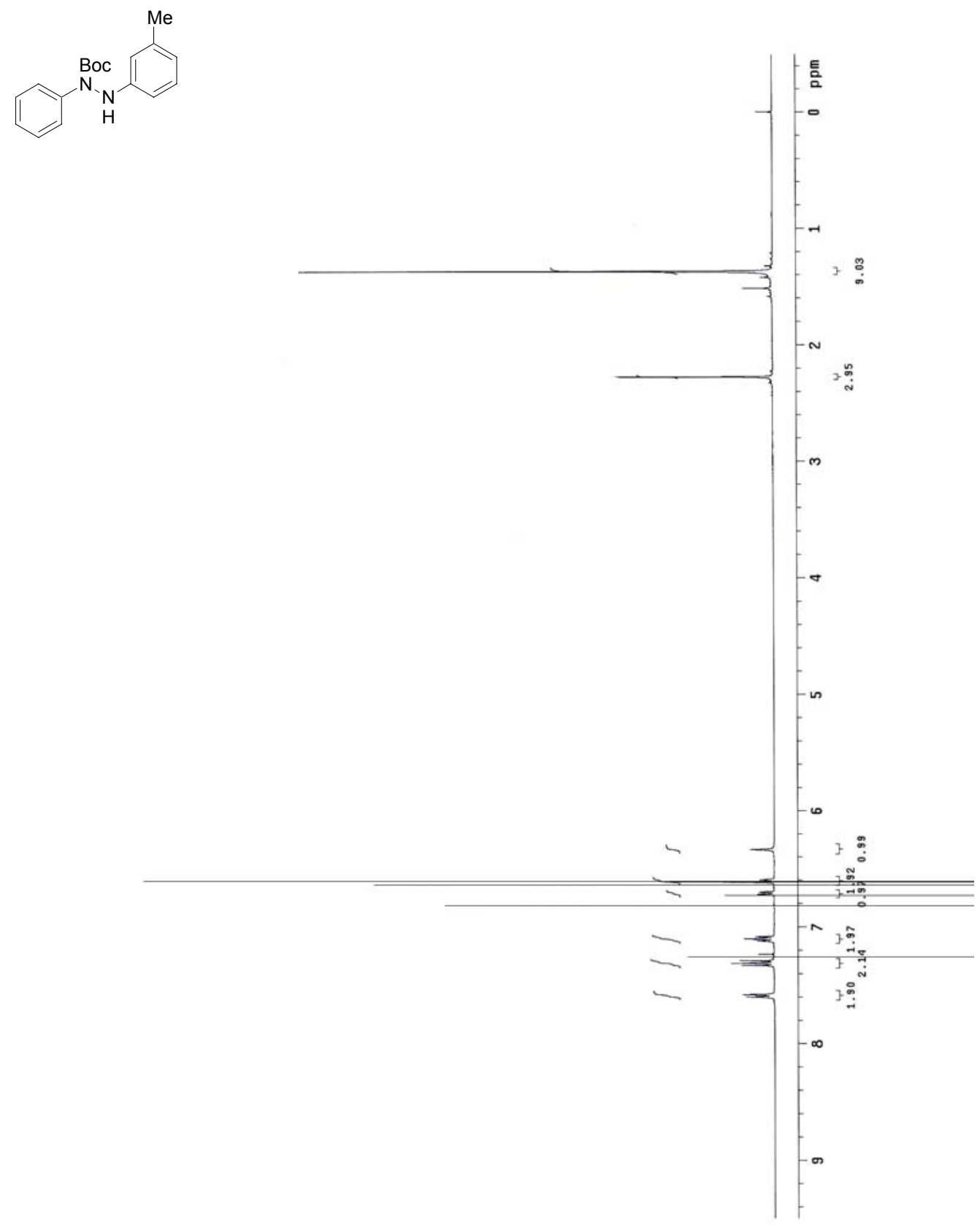

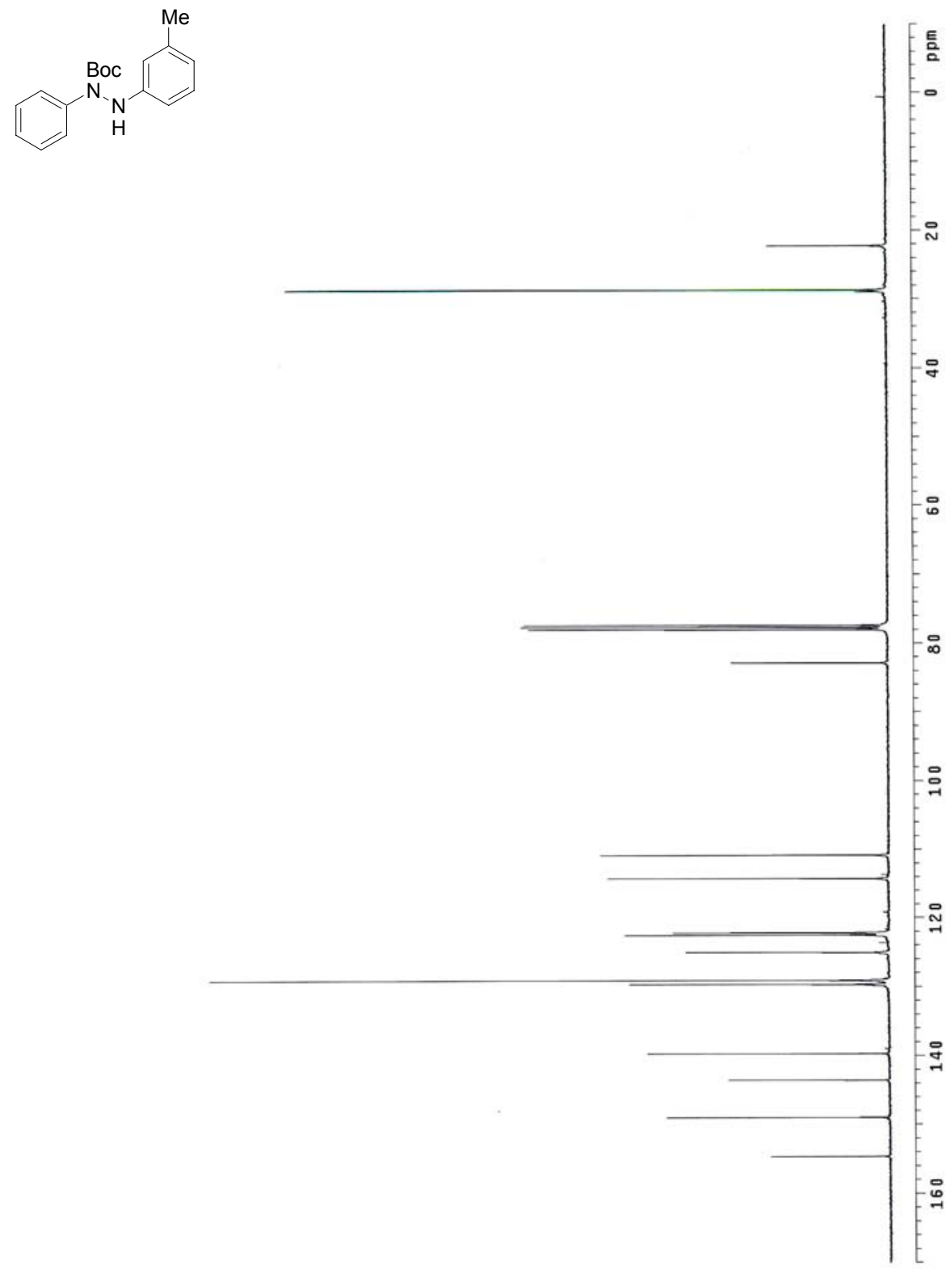

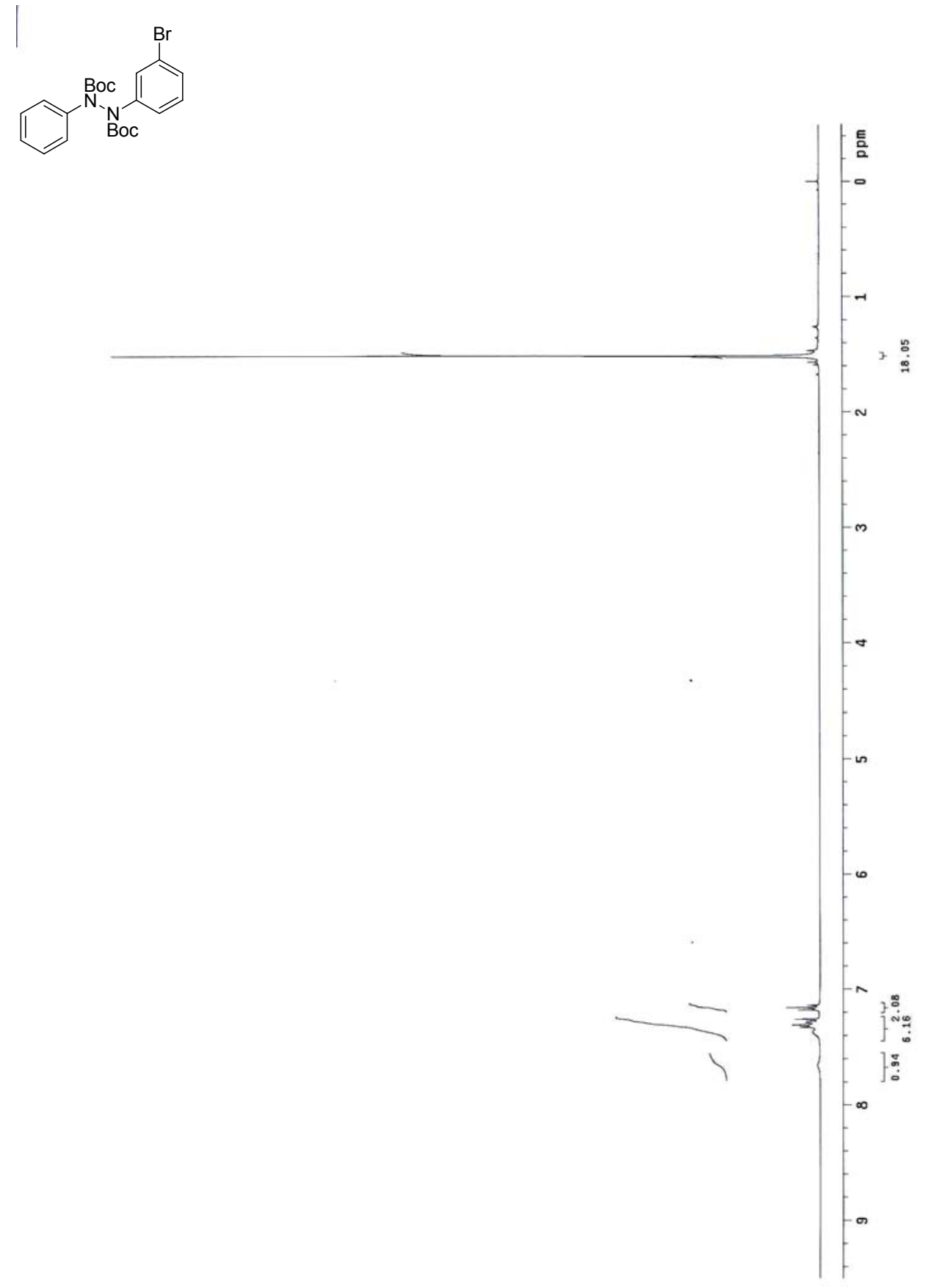


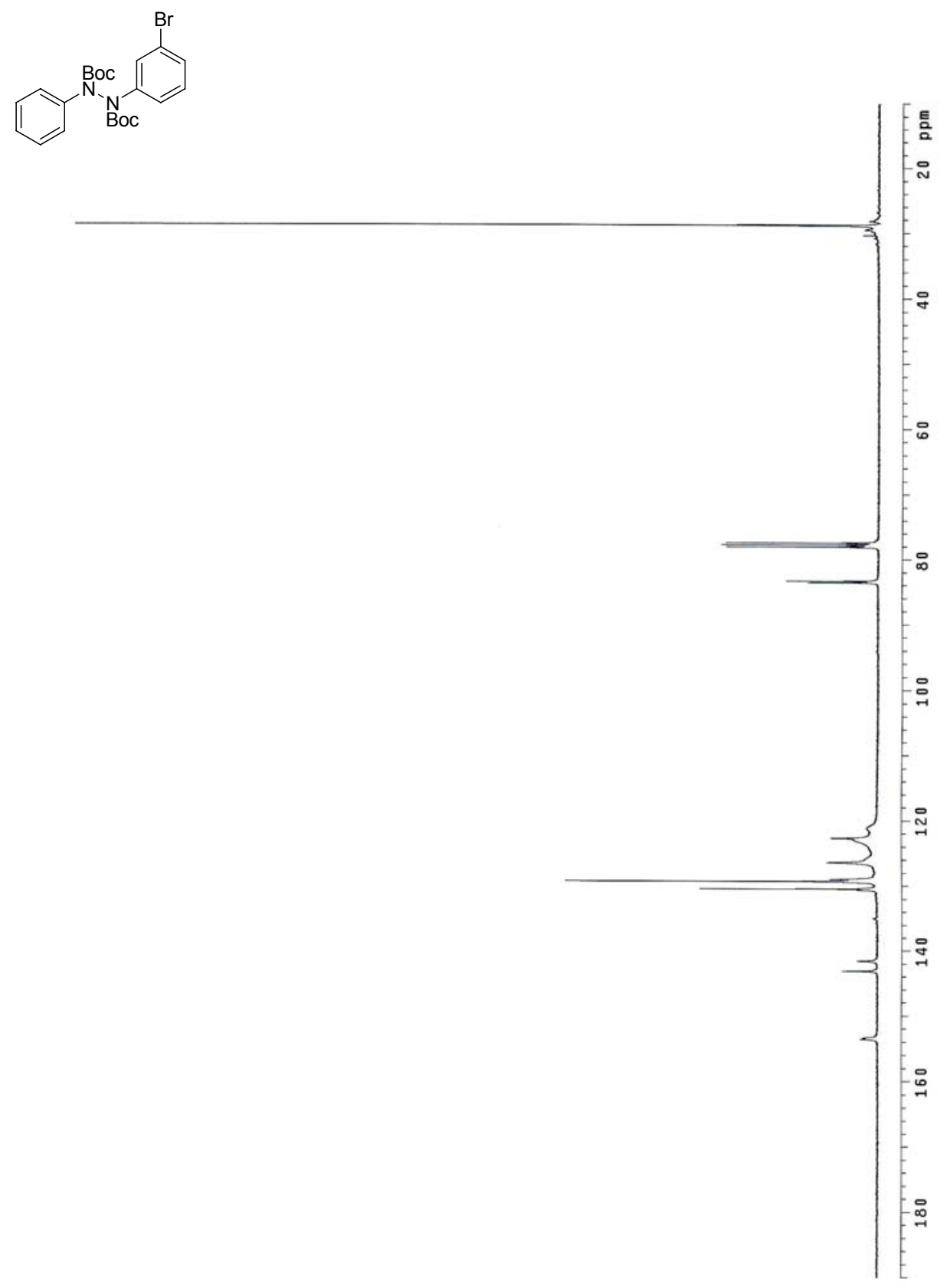



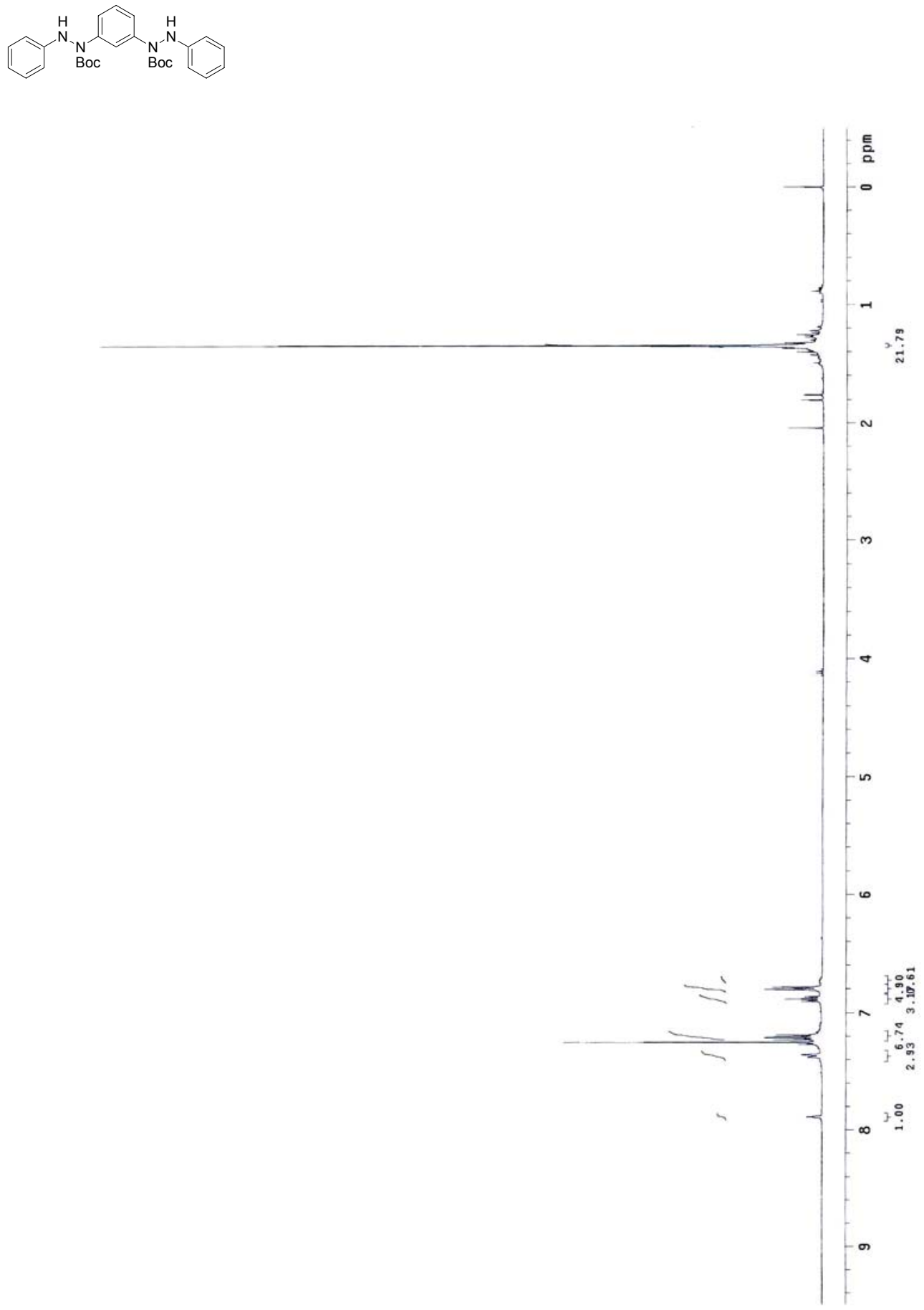

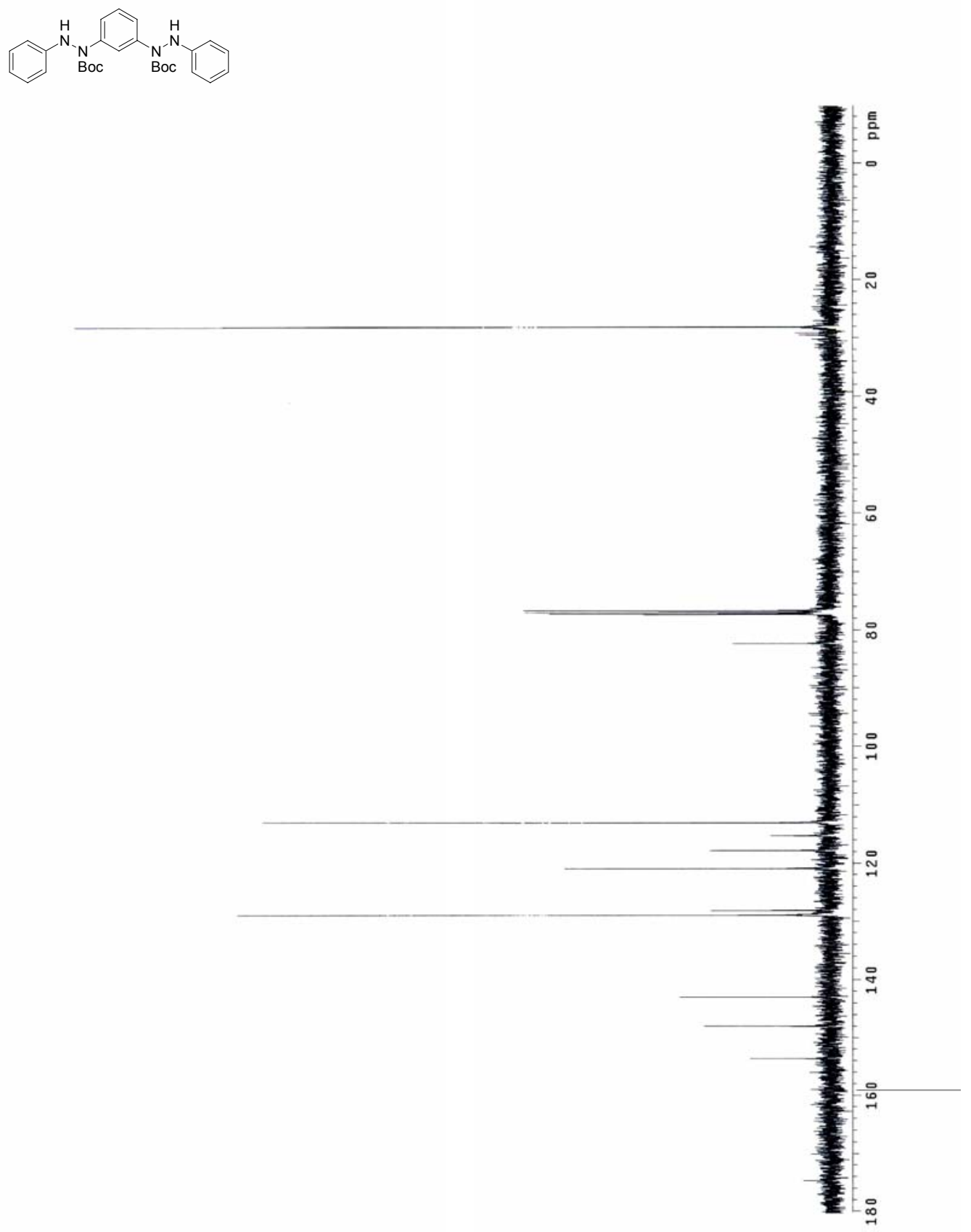
$\underbrace{M e}_{B \text { Boc }}$

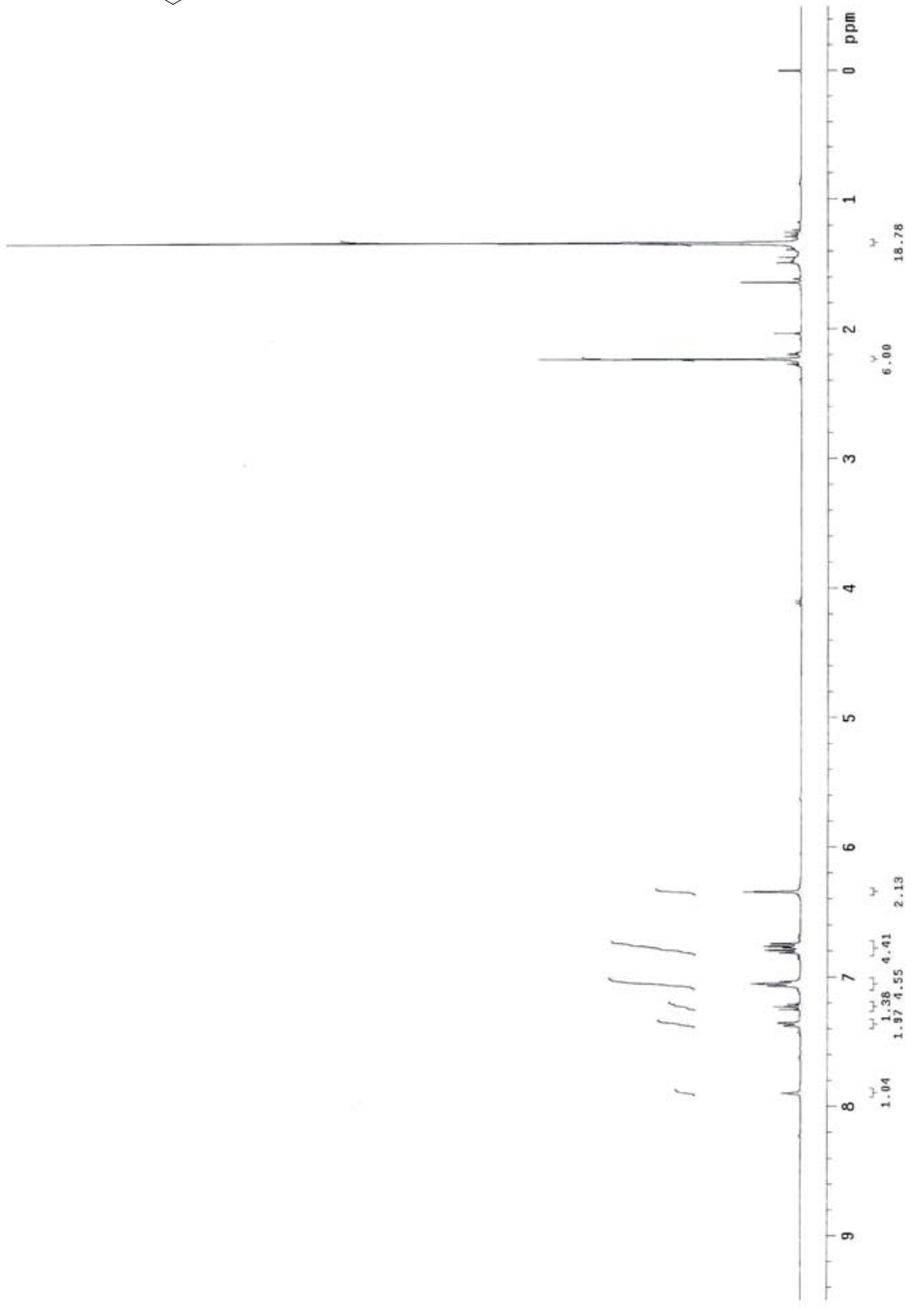




$$
\text { Boc }
$$

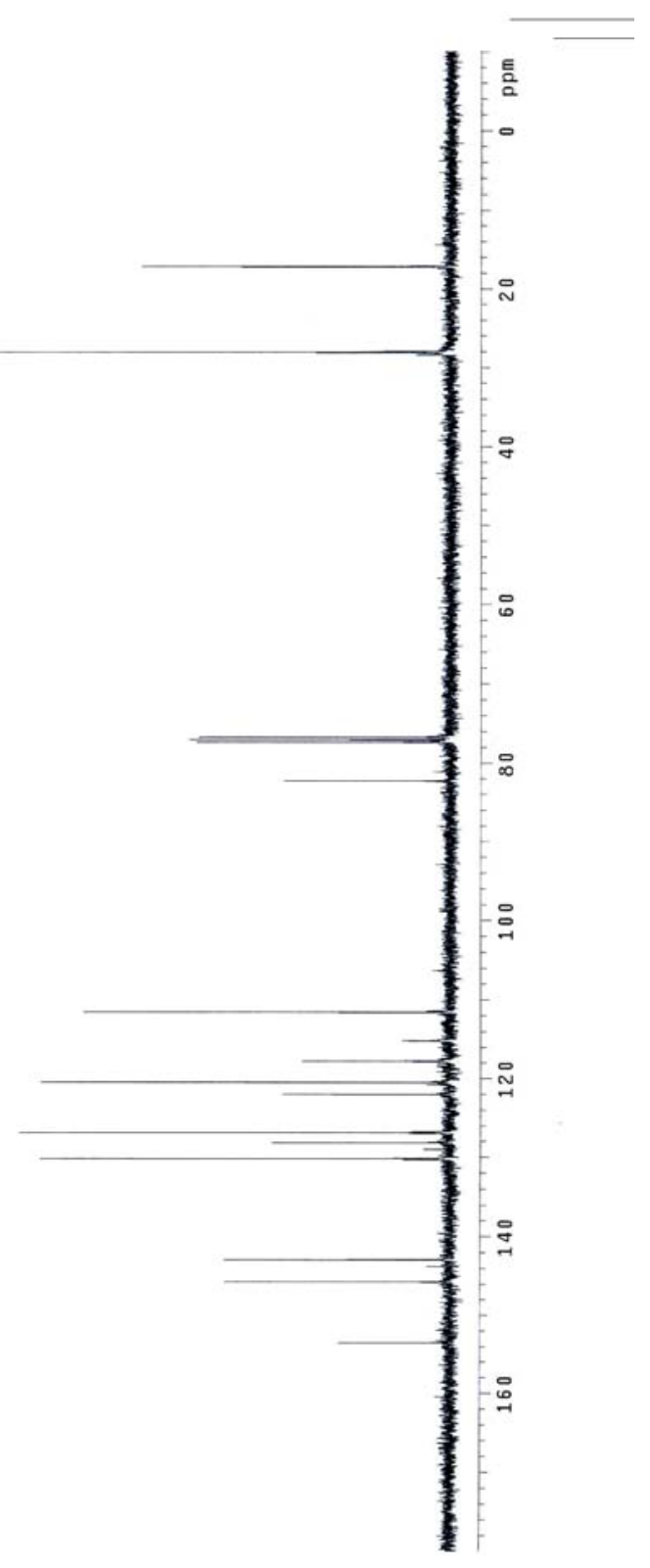


$\underbrace{B_{B o c}^{M}}_{M e}$

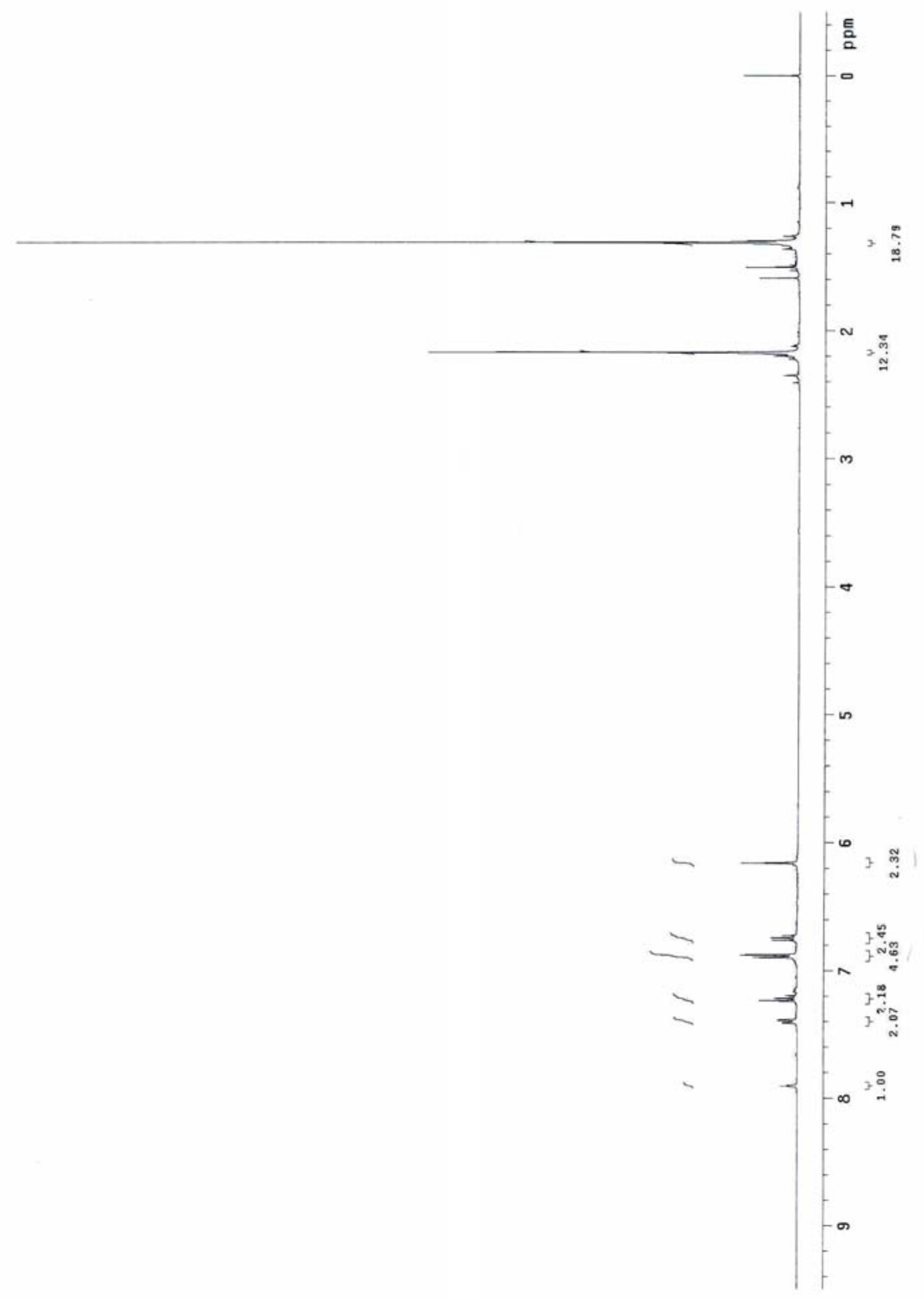


(1)

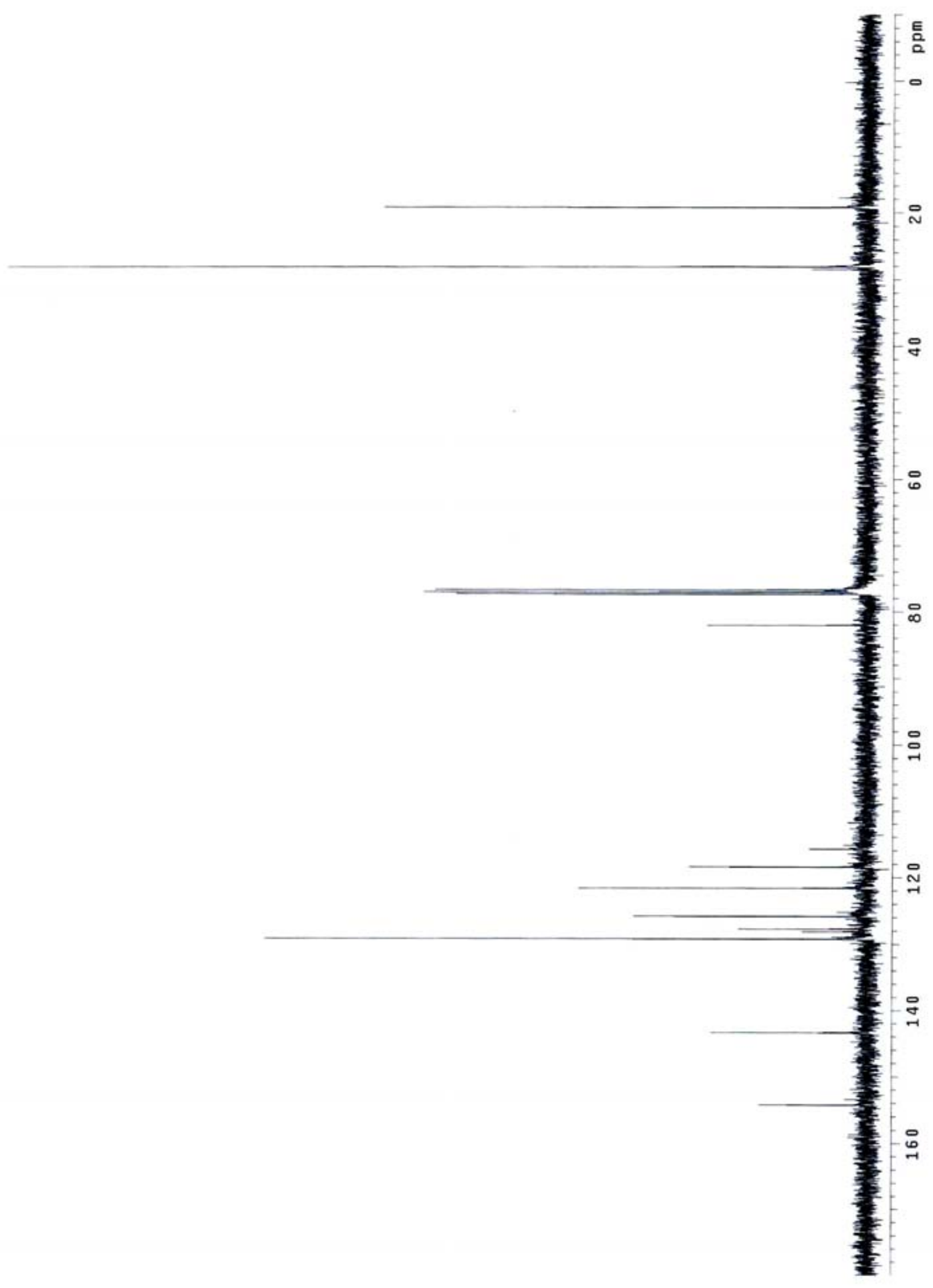




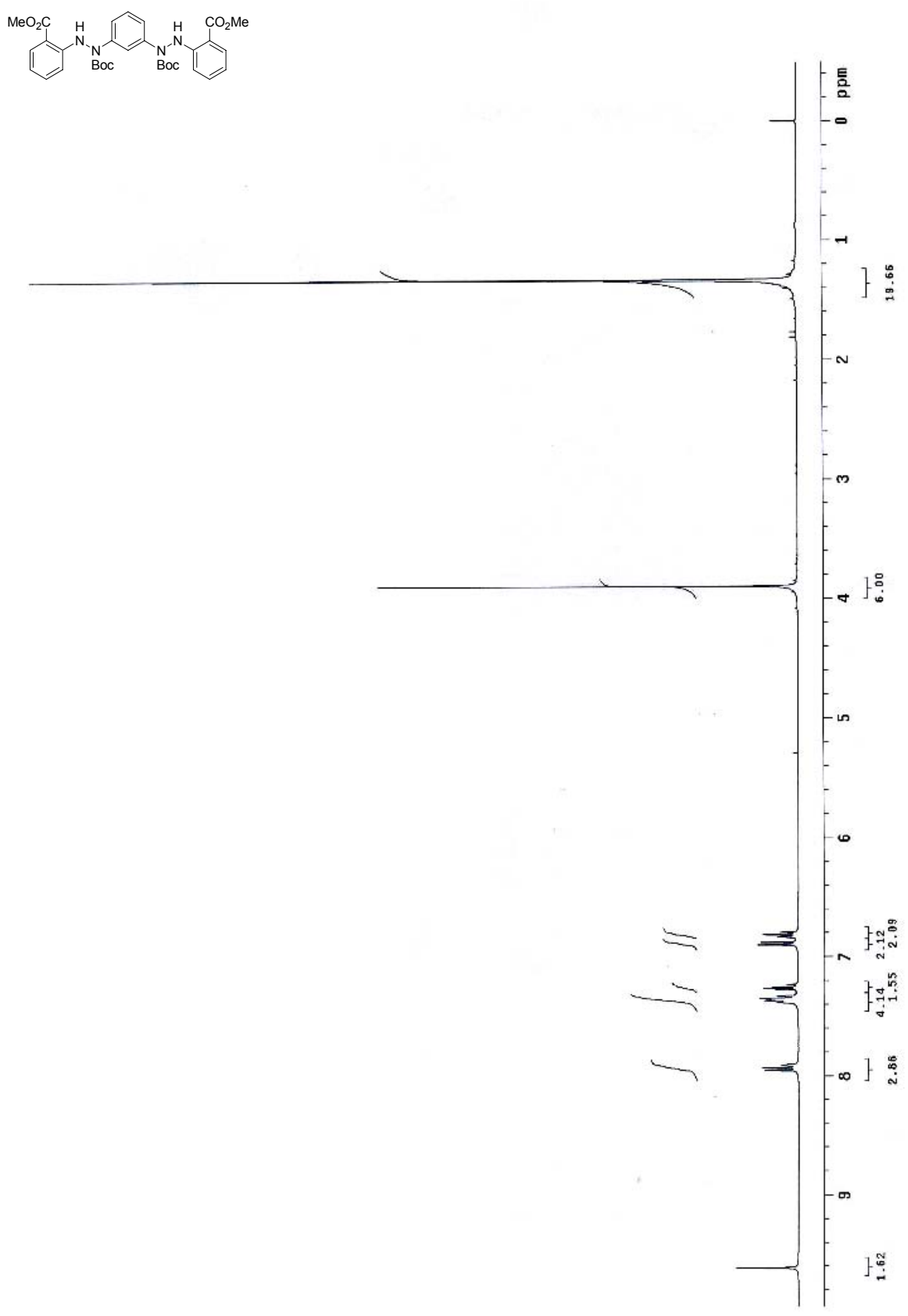


Boc

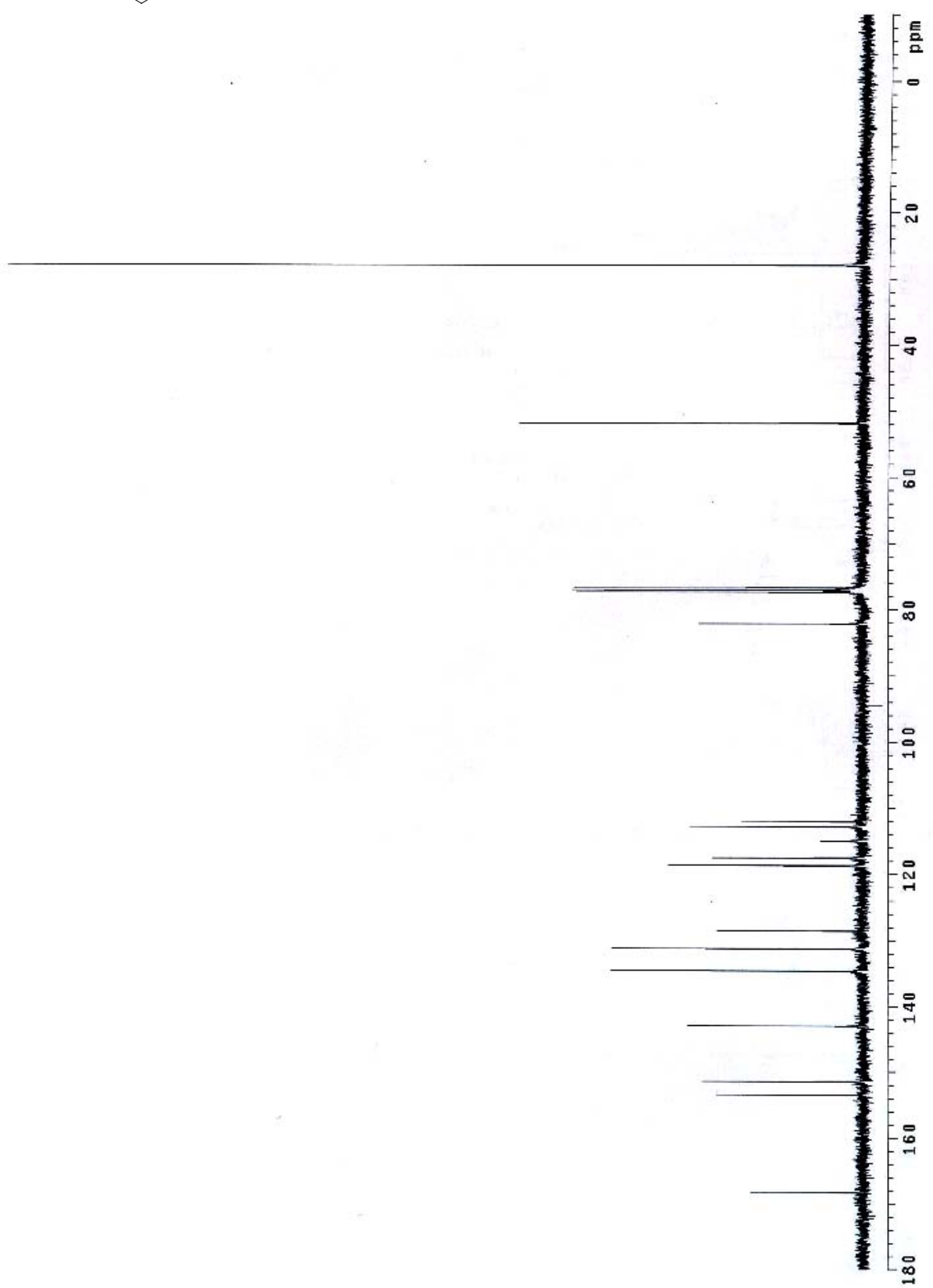



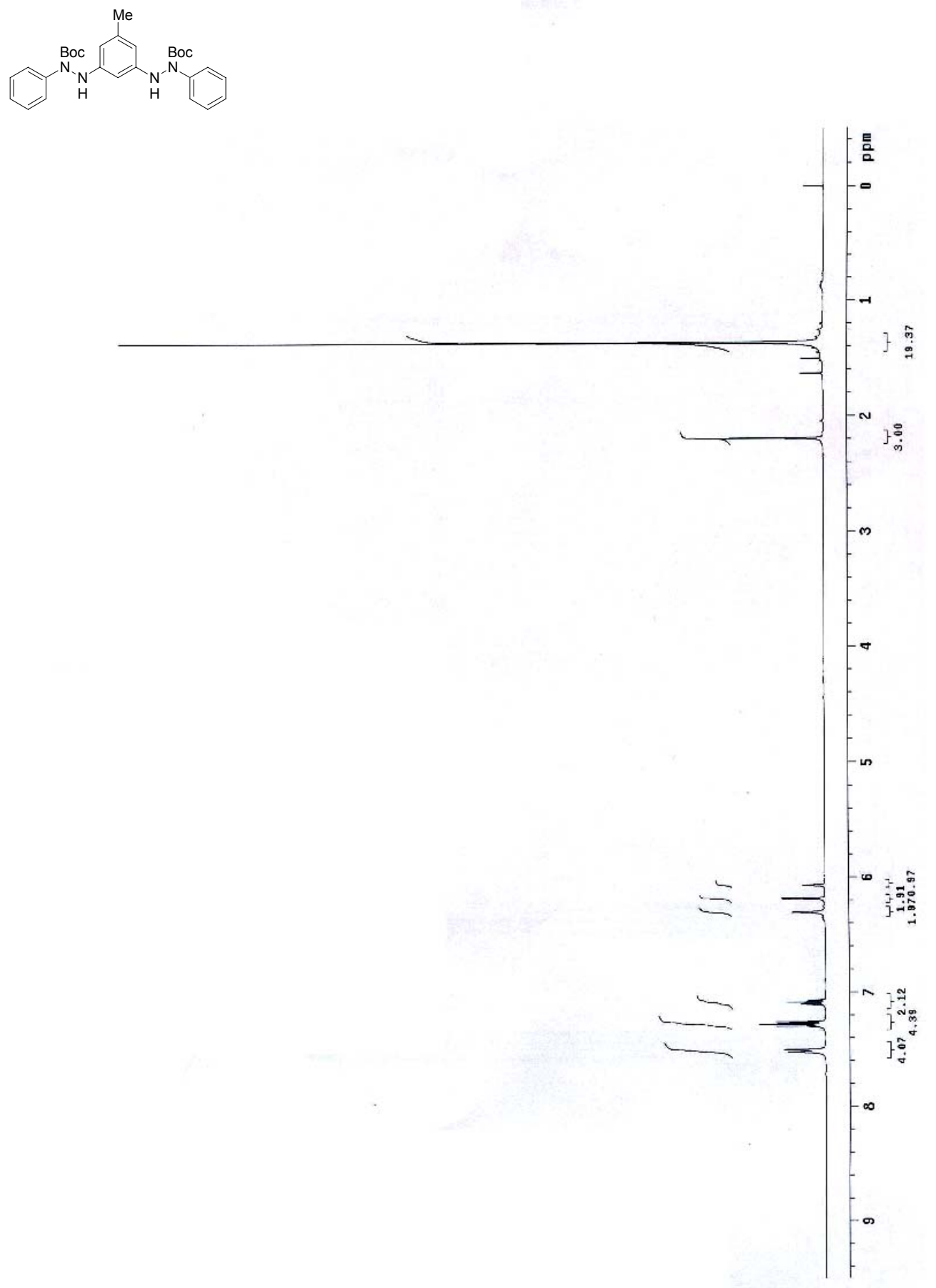

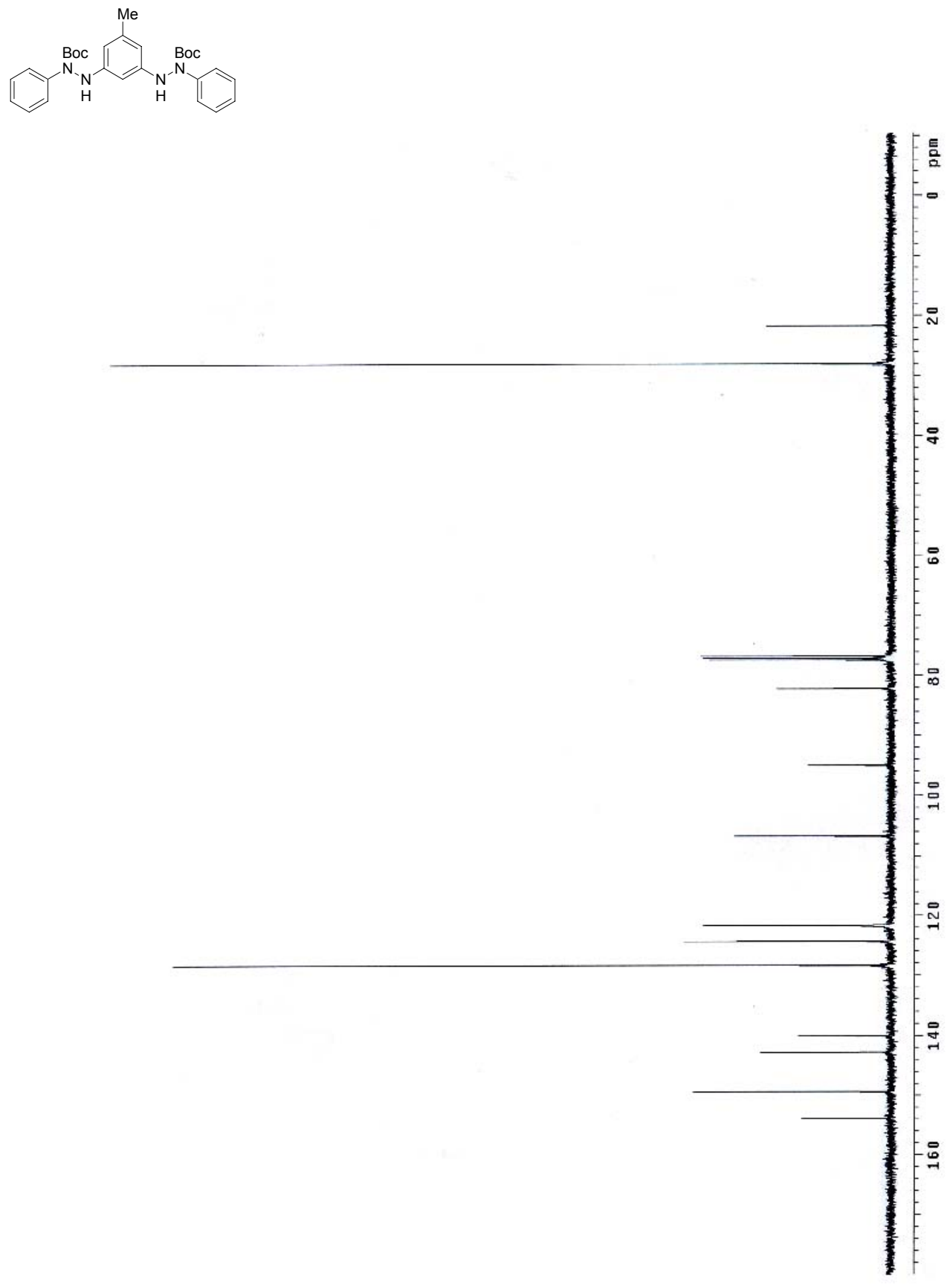

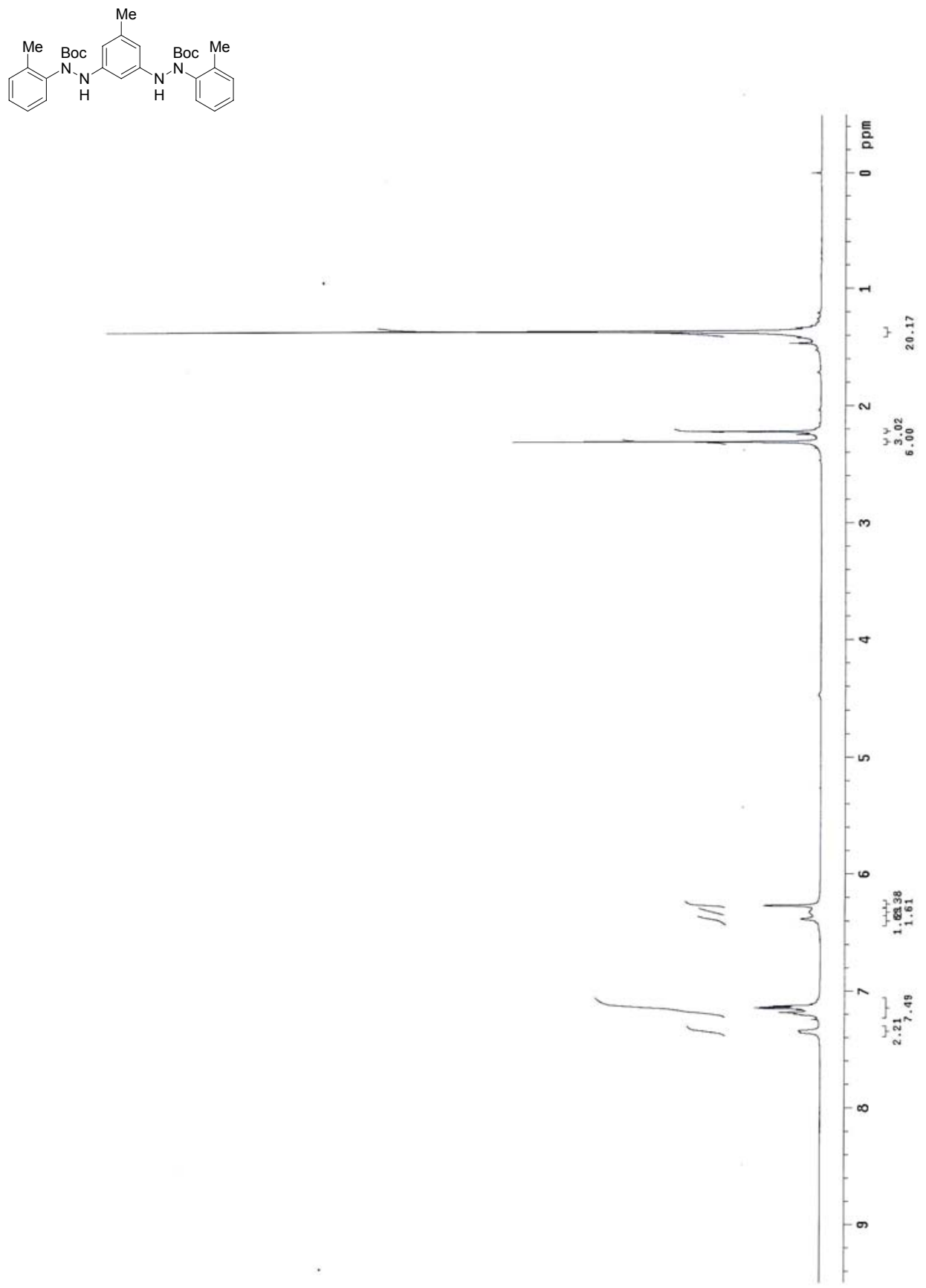

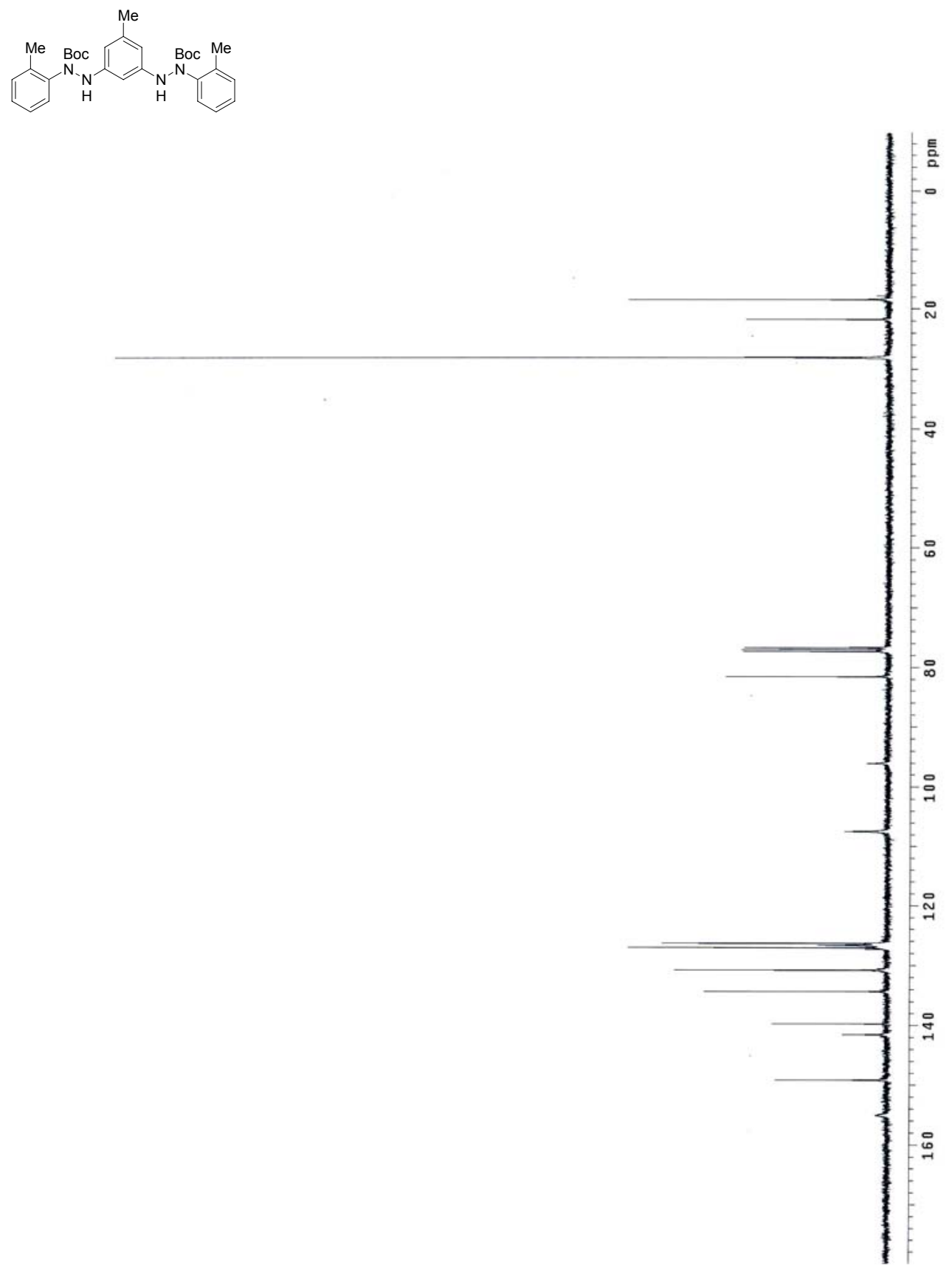

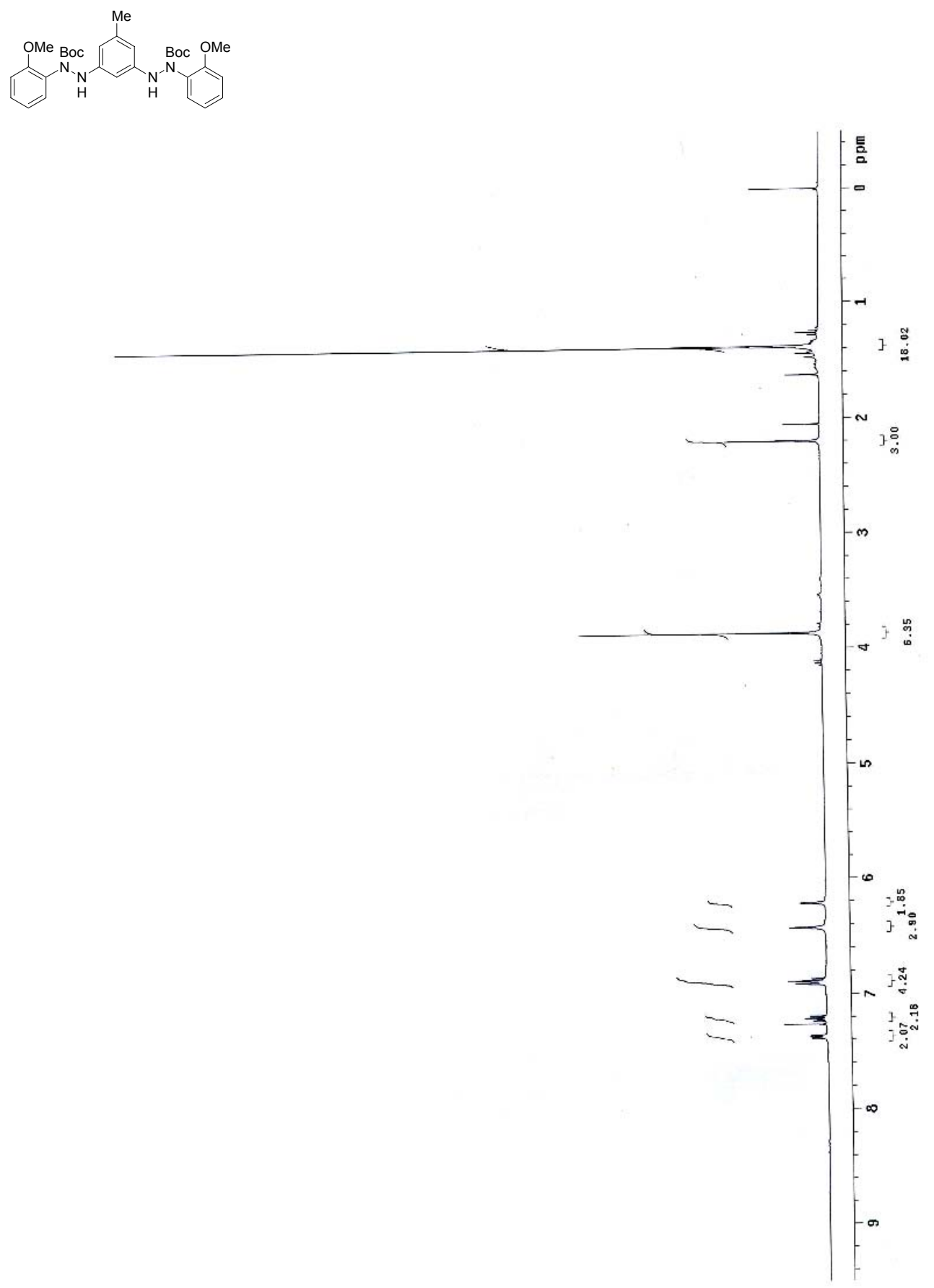

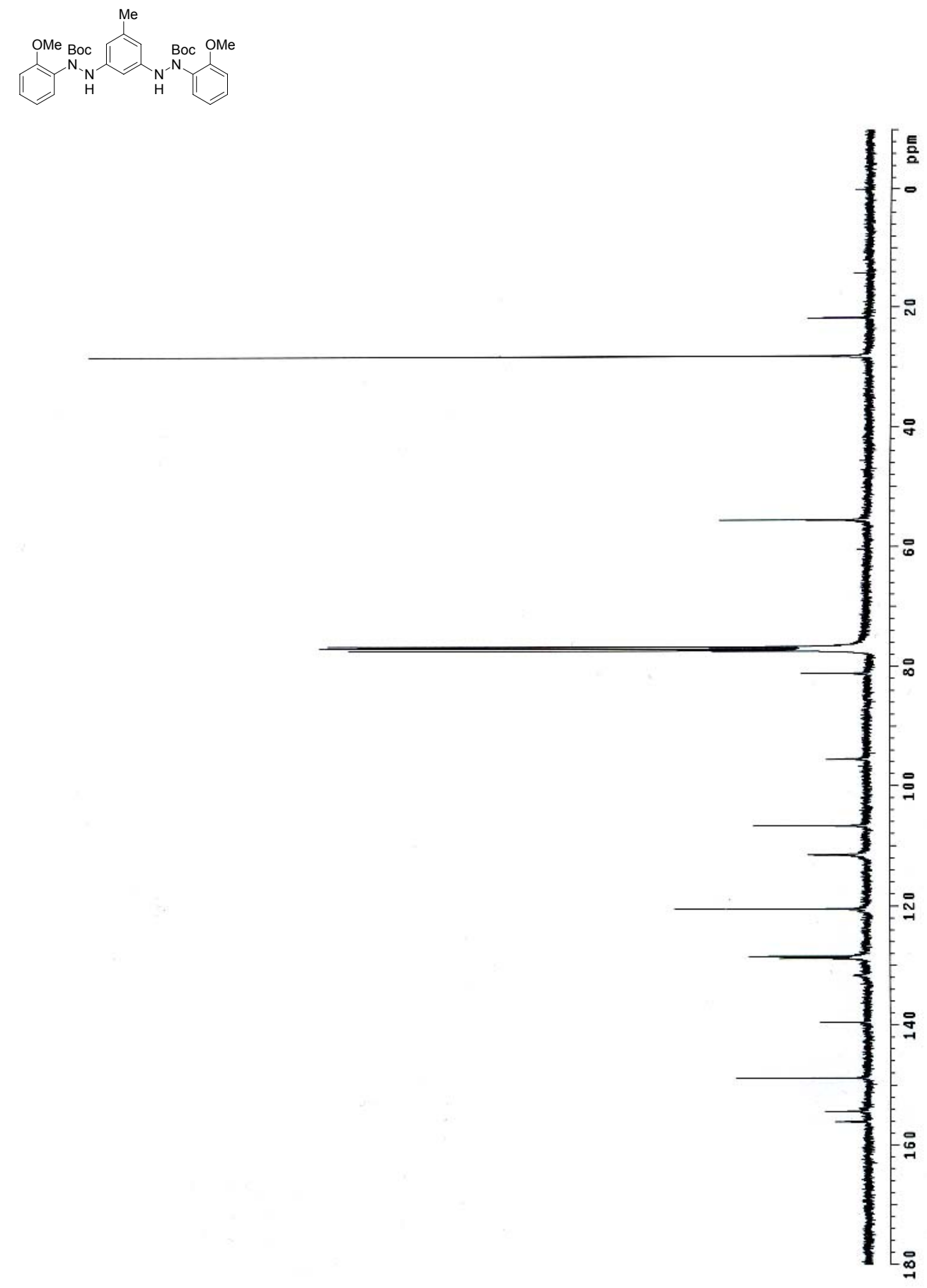

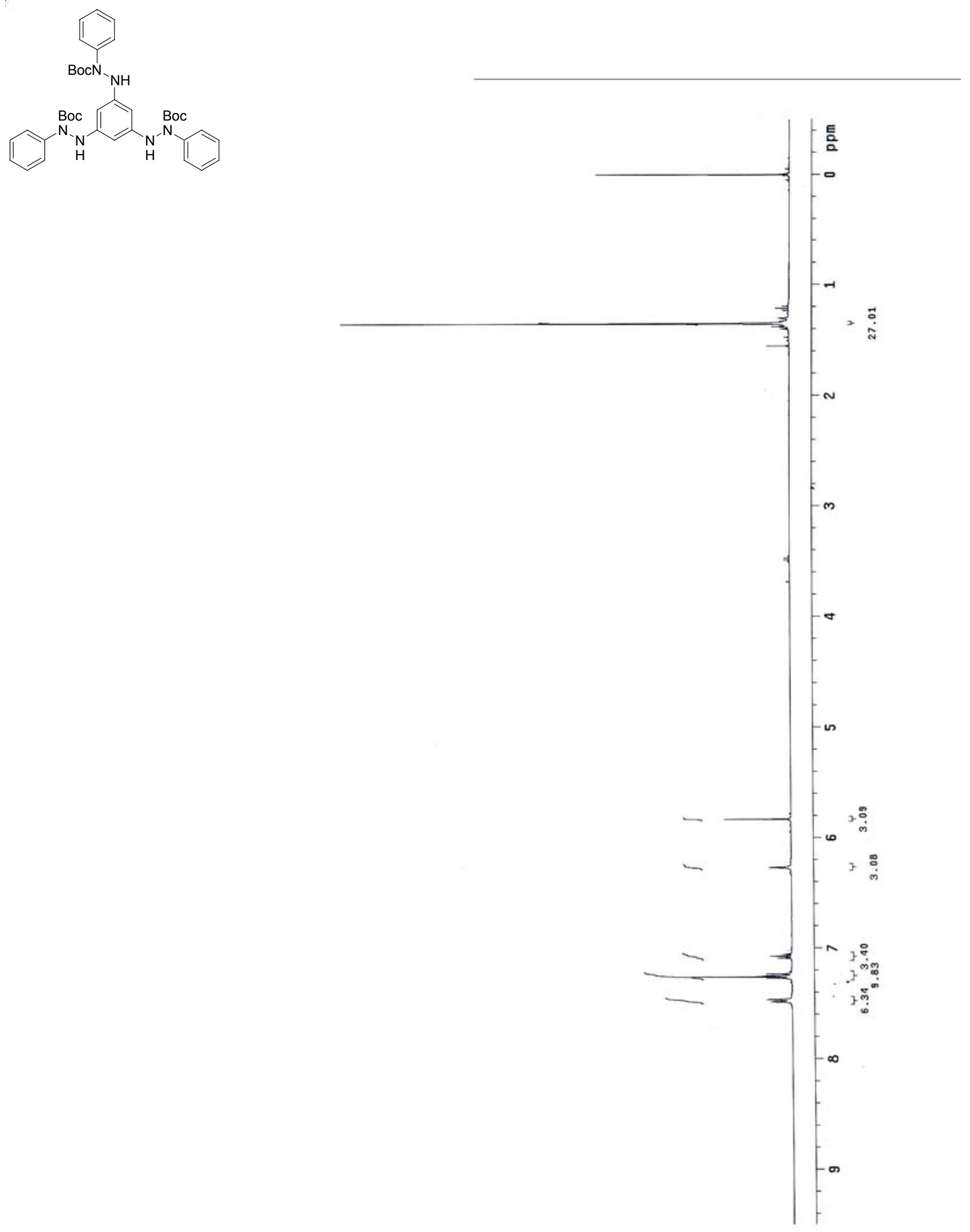


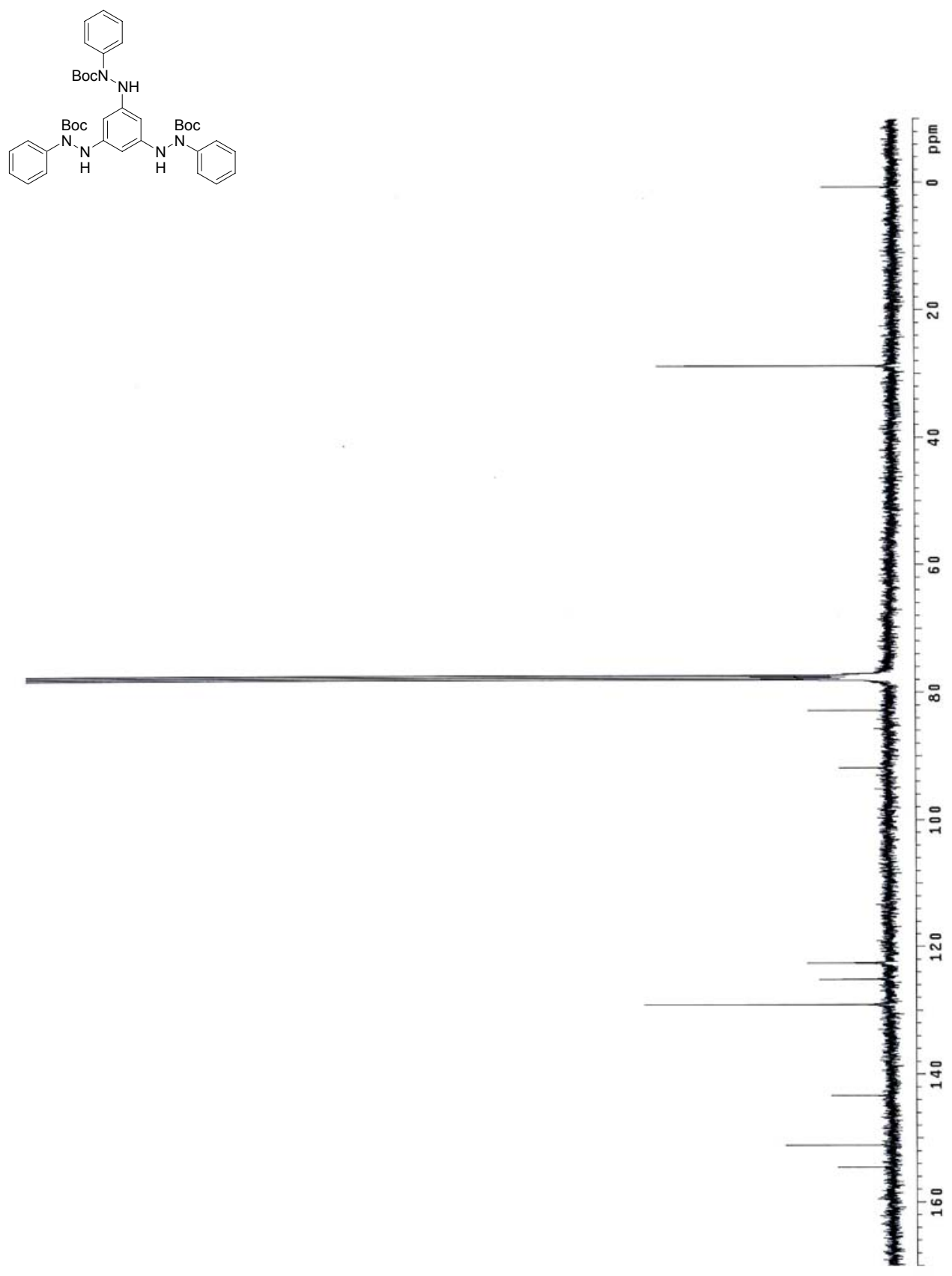



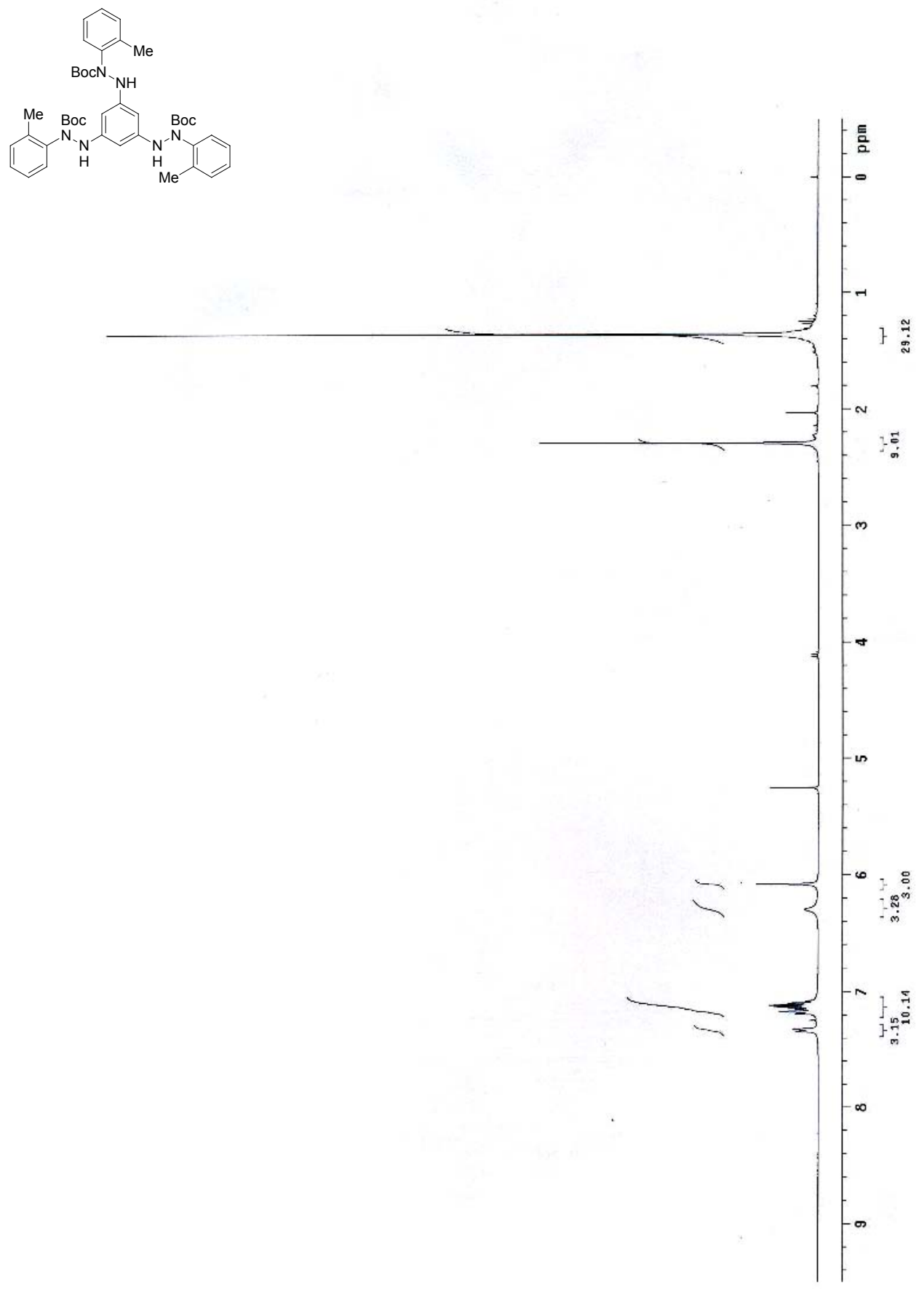

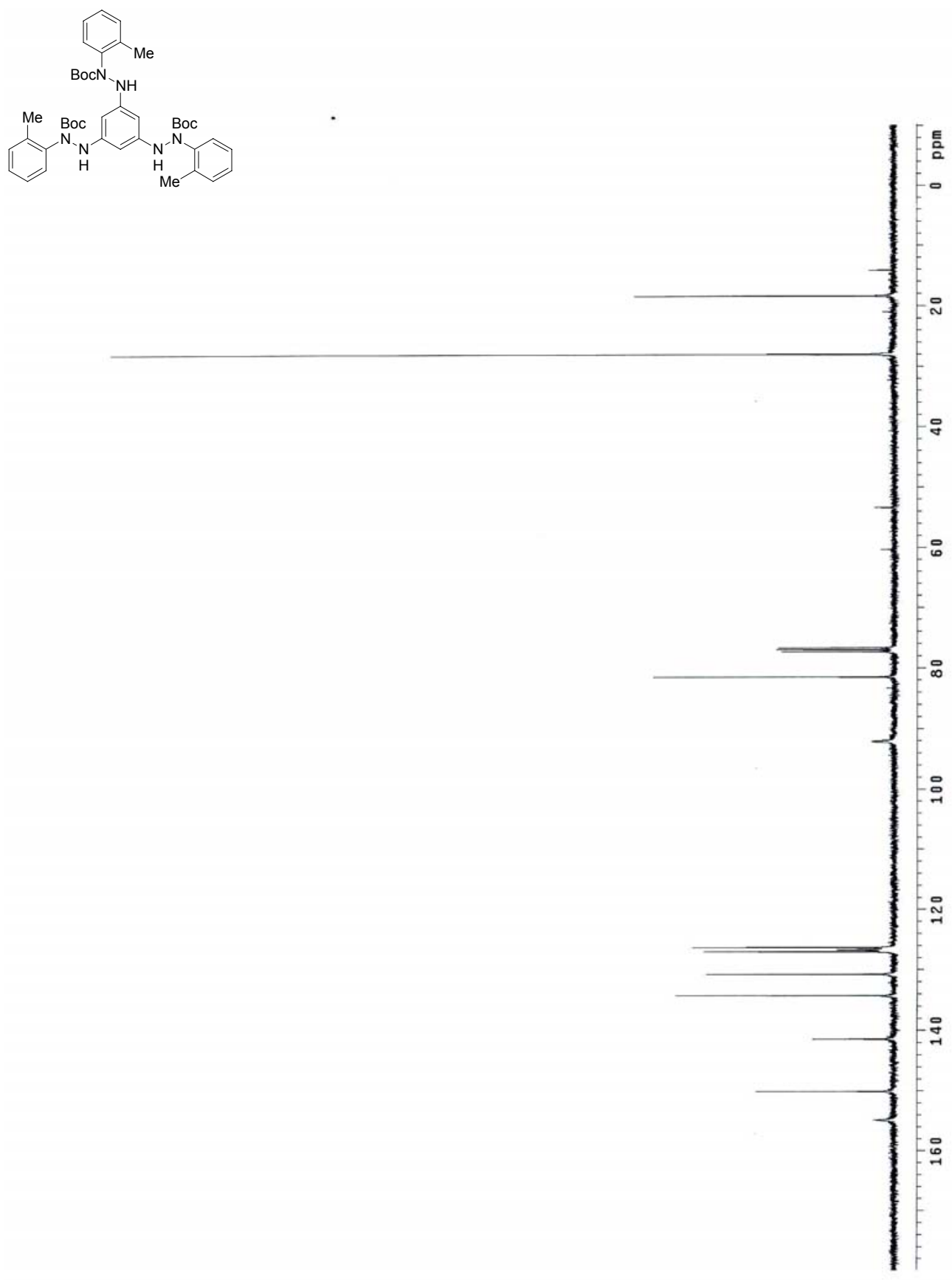


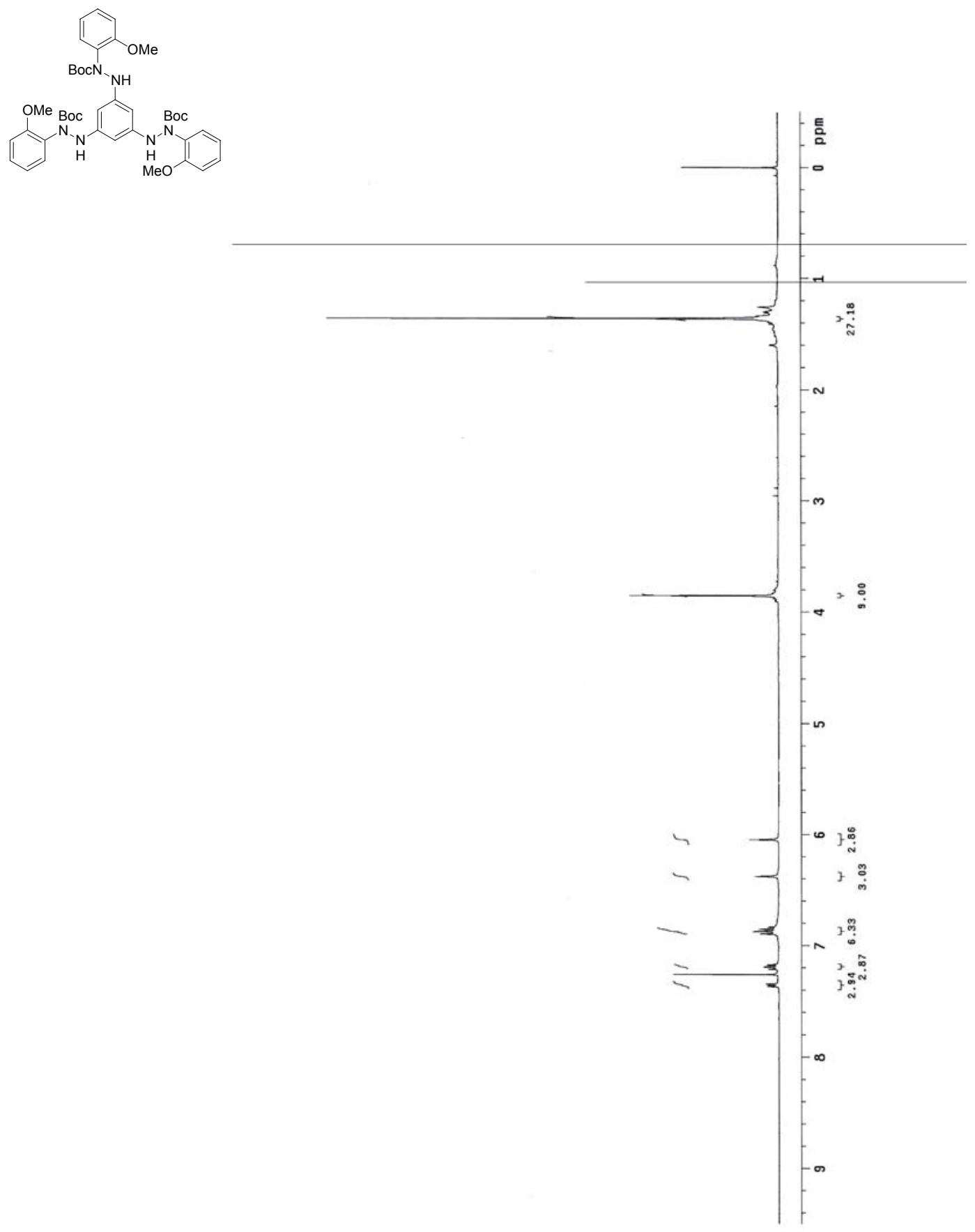



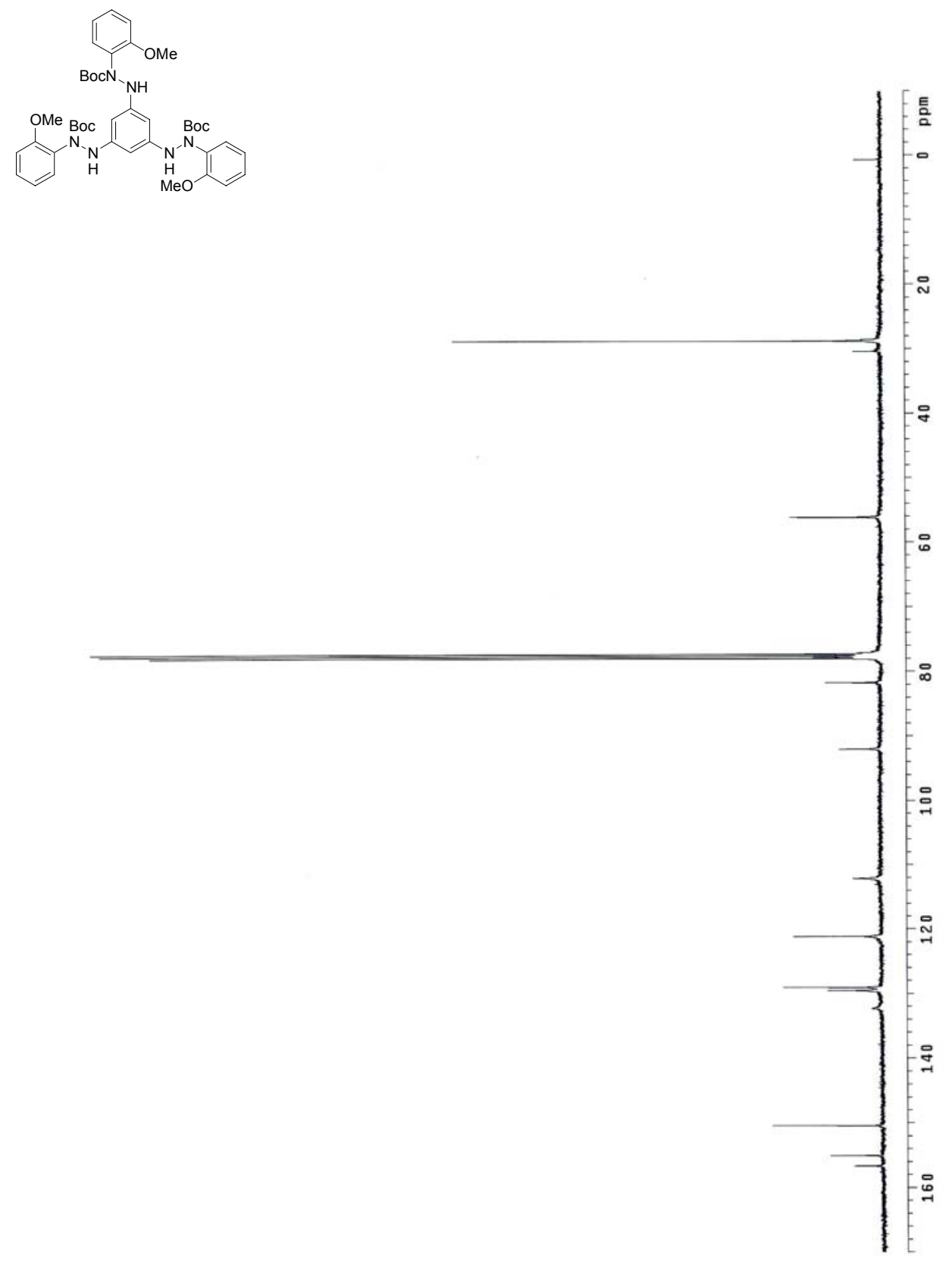

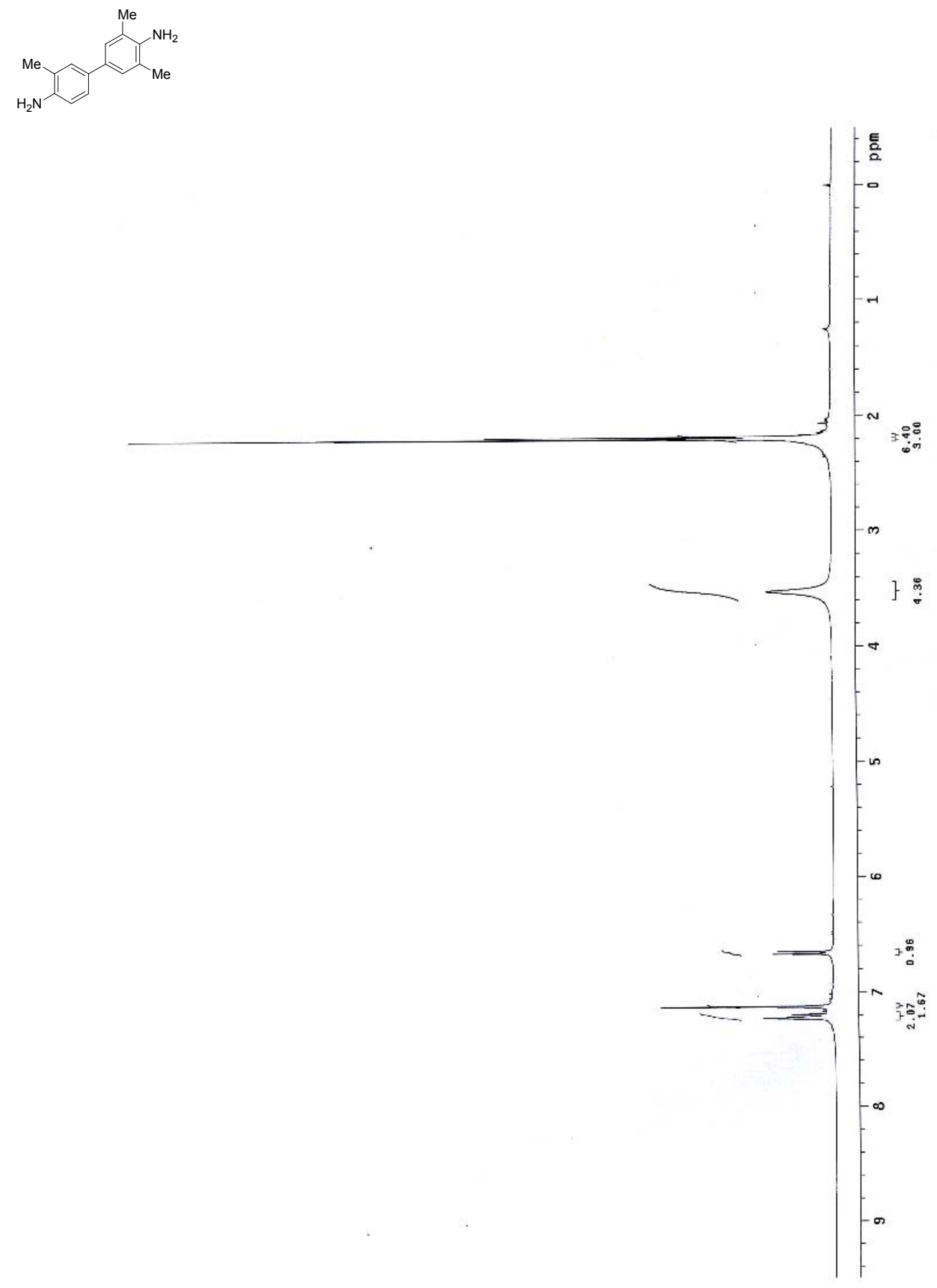

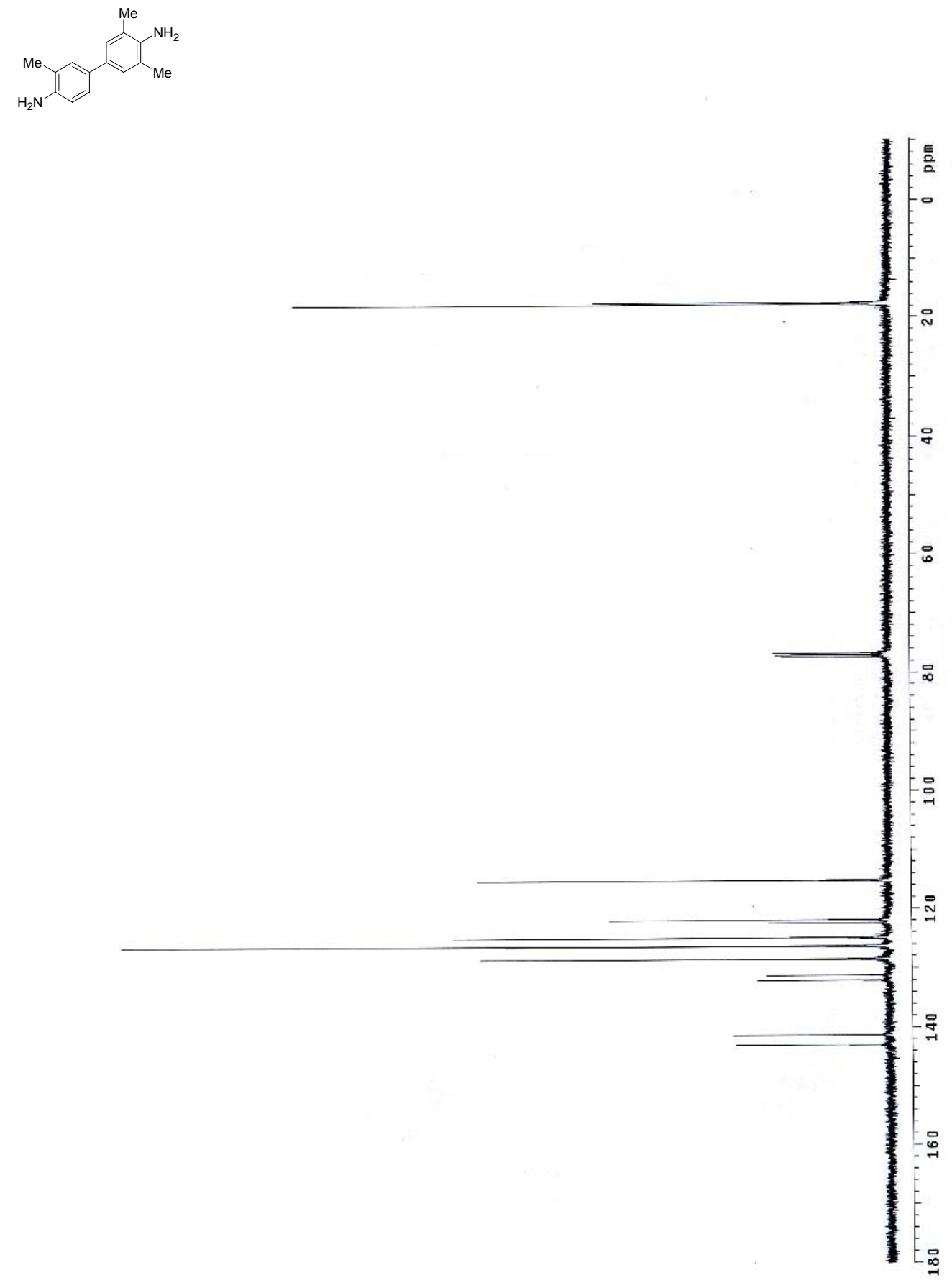

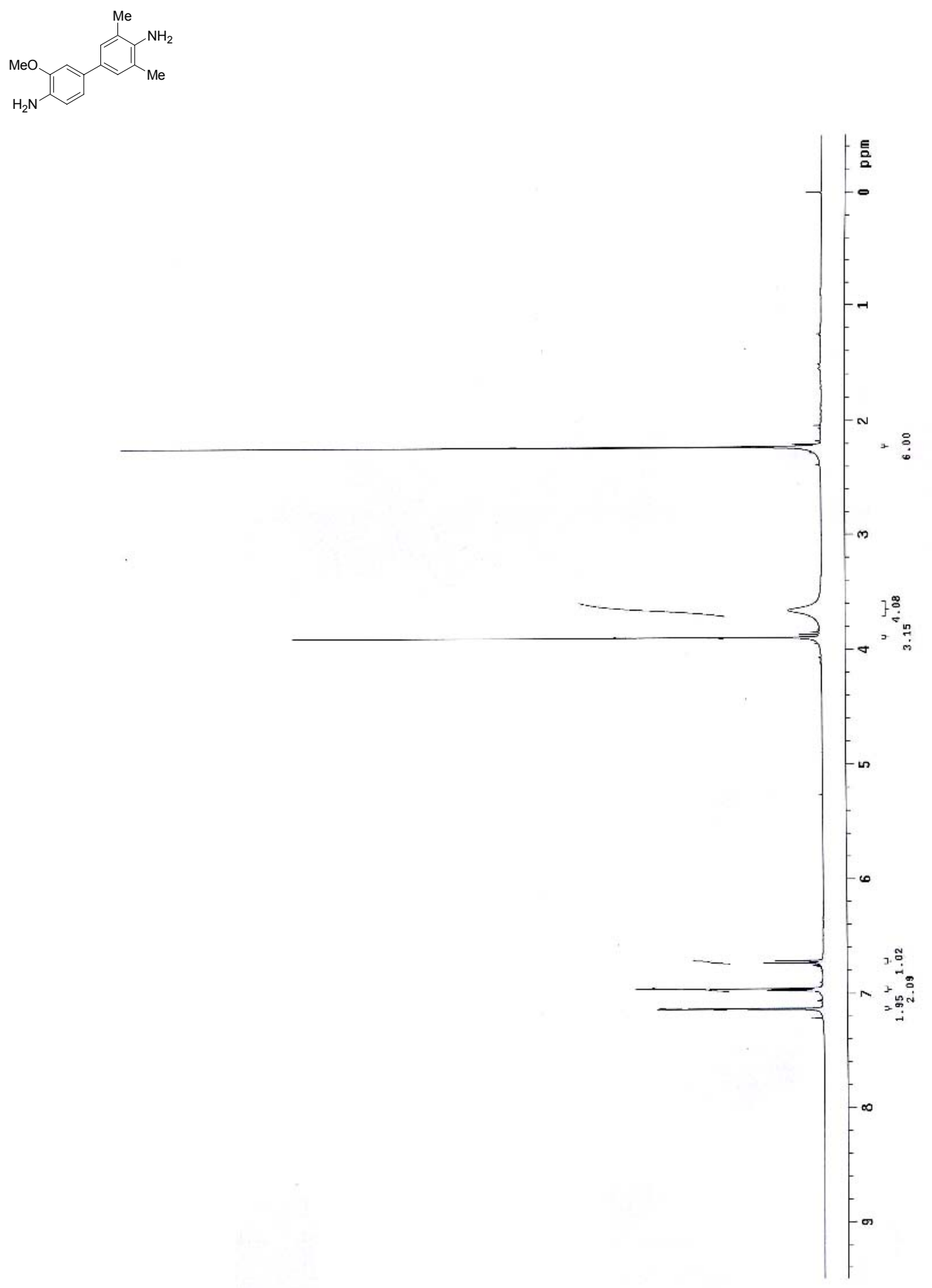

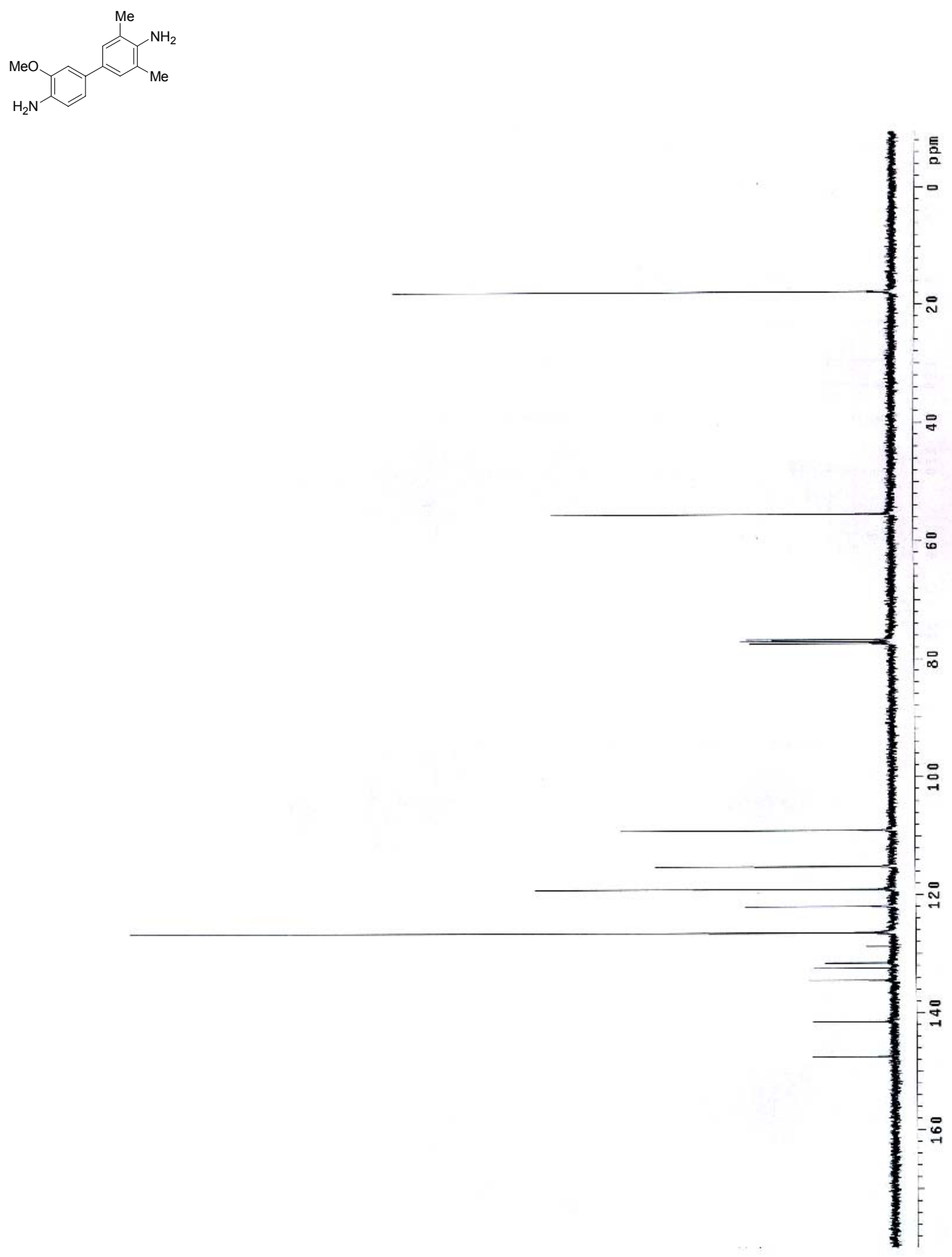

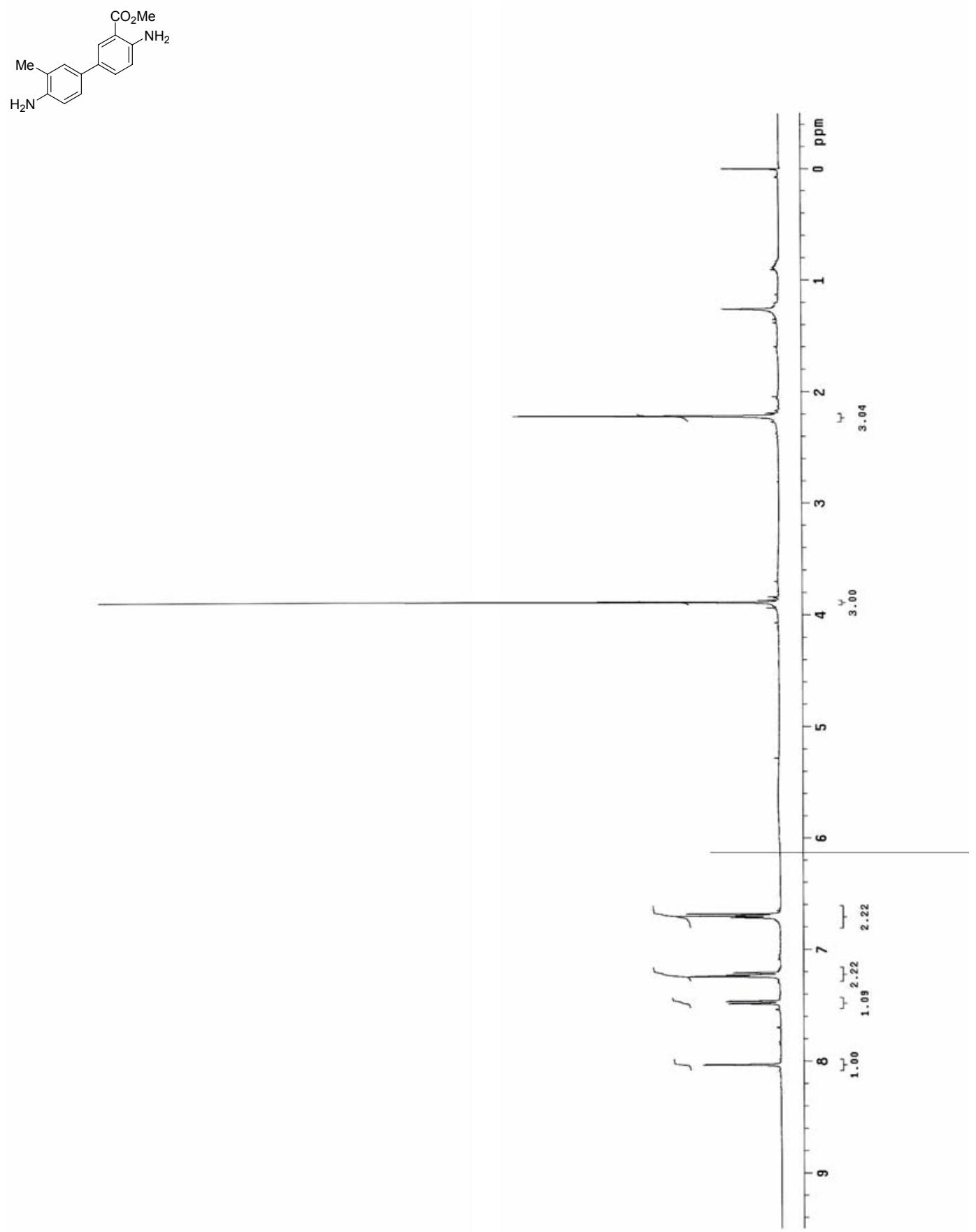

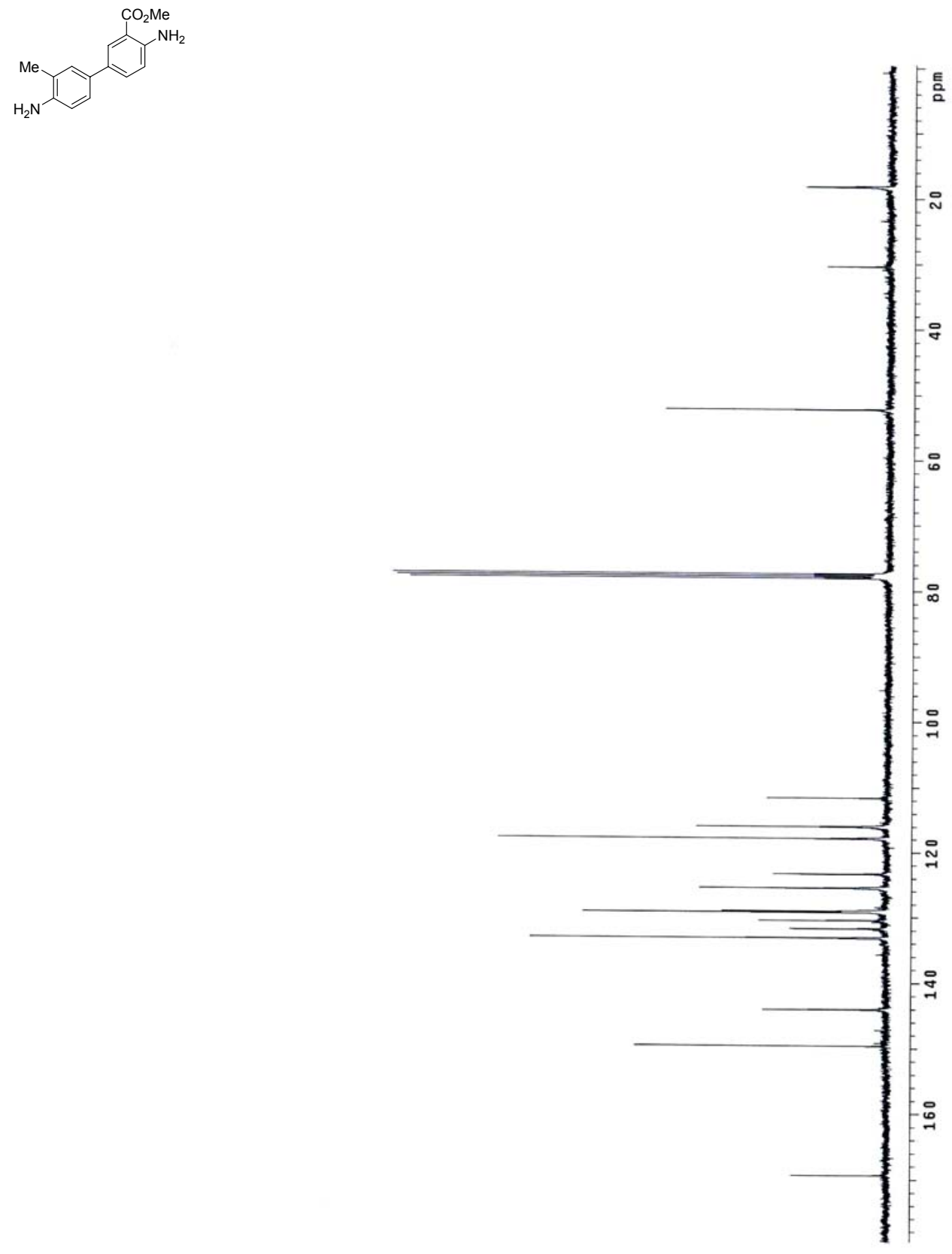

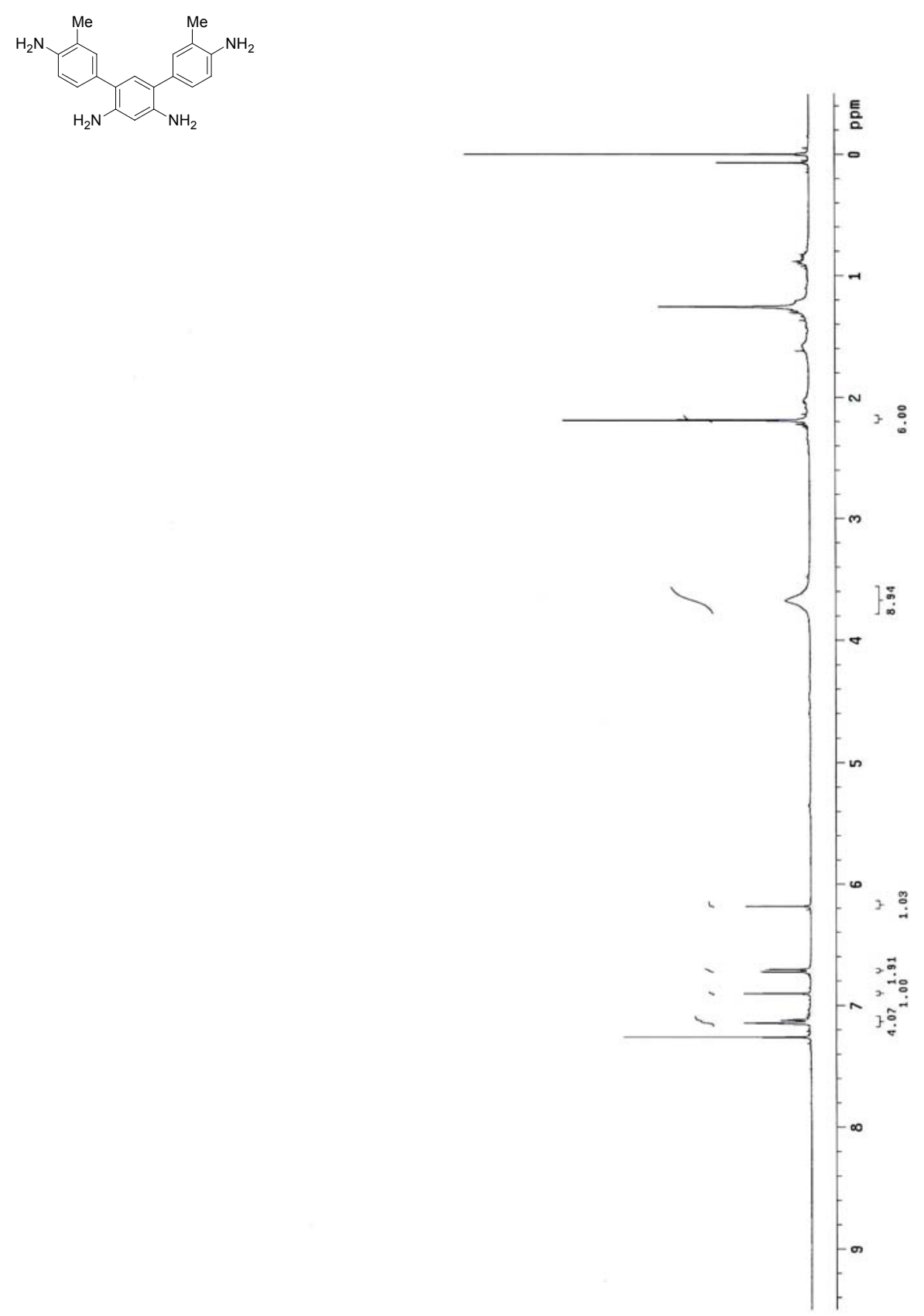

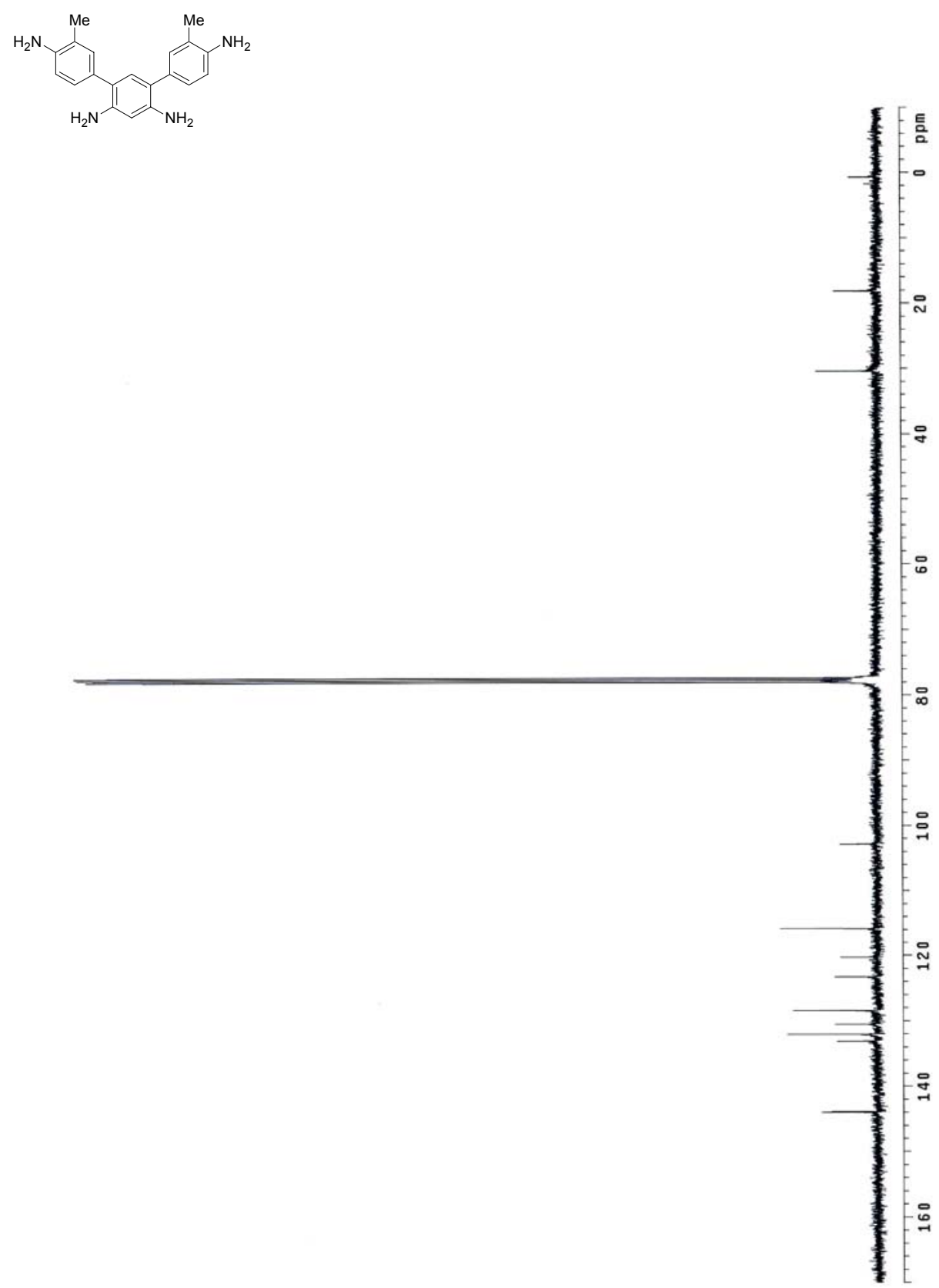

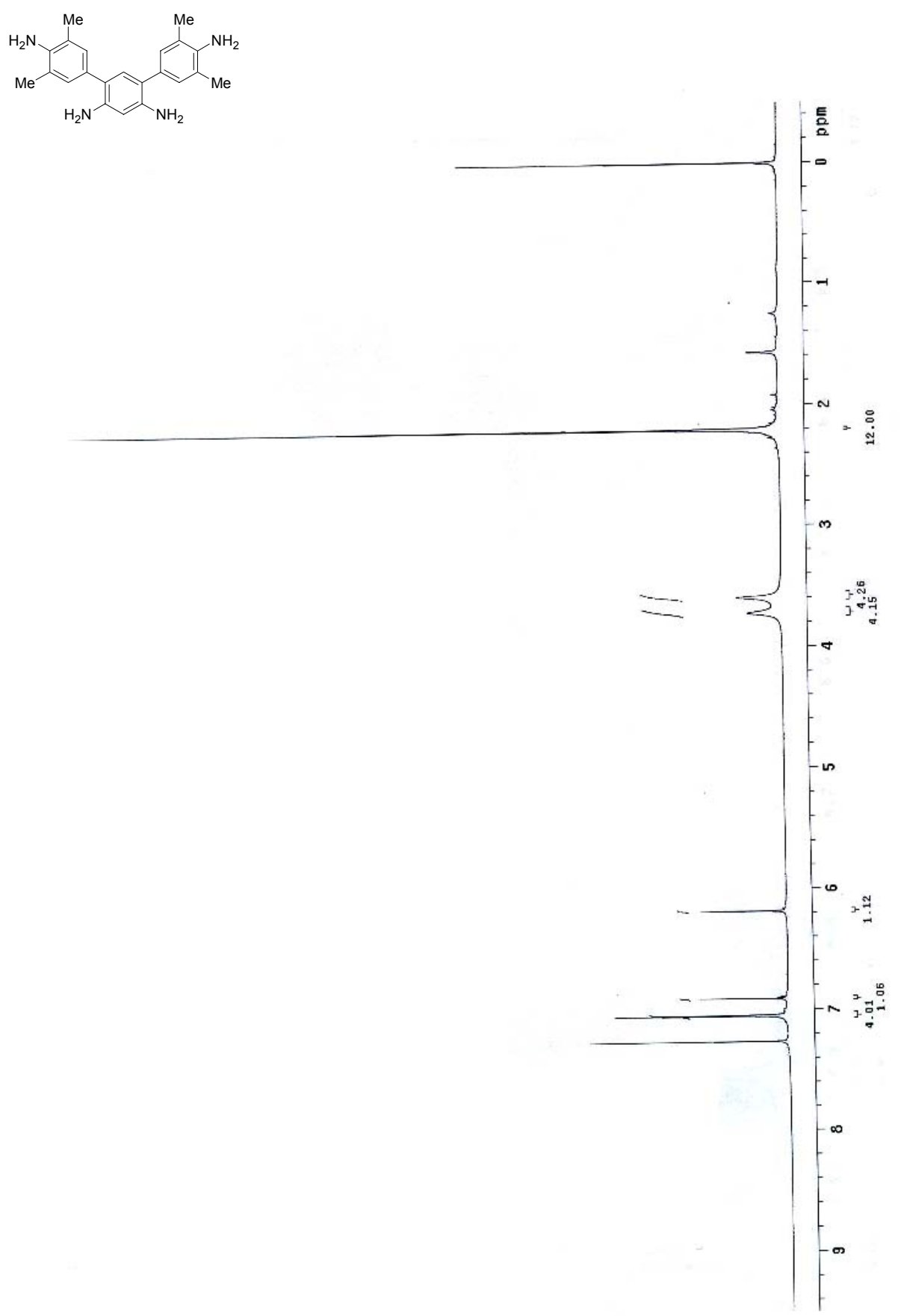

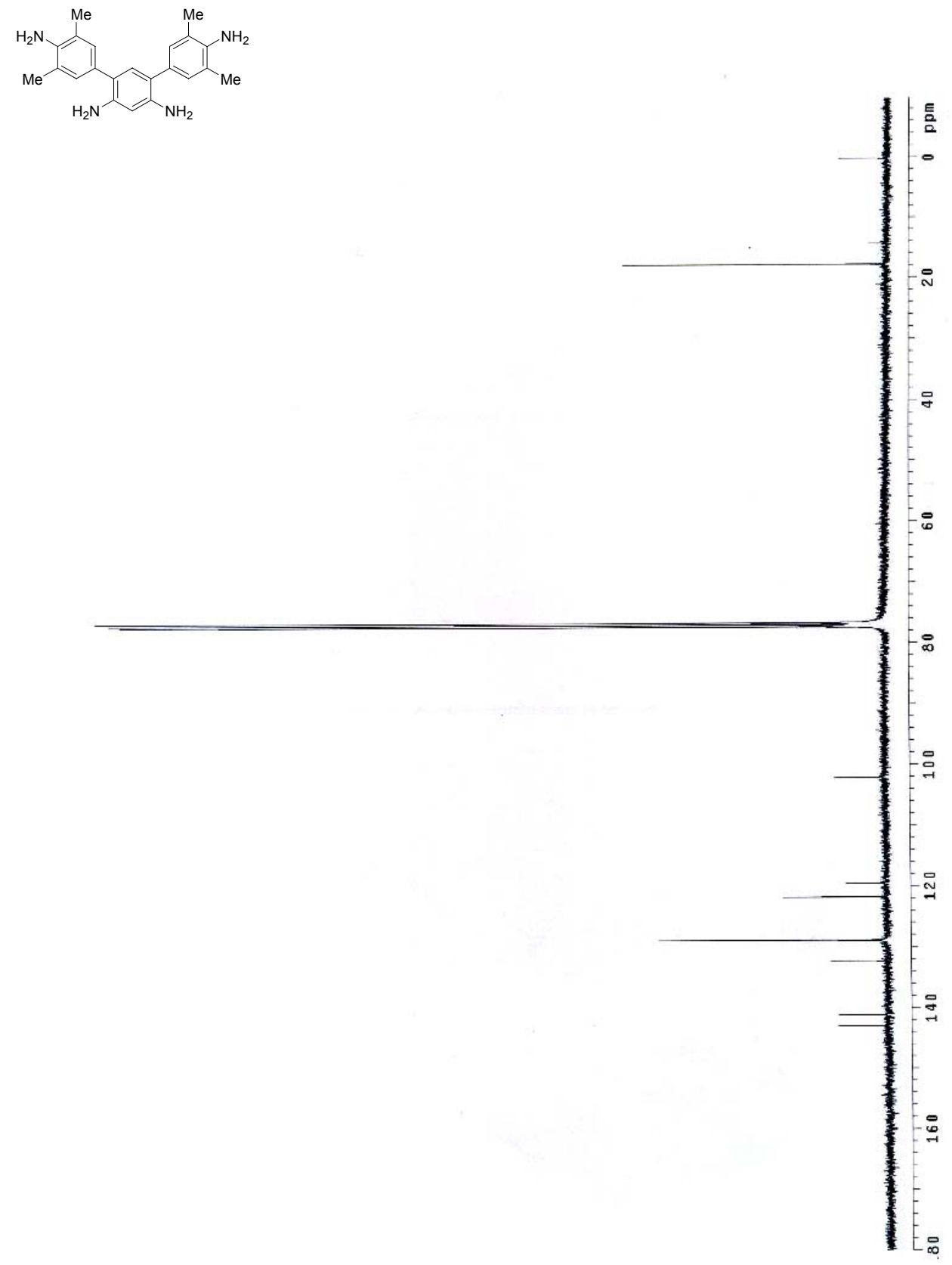


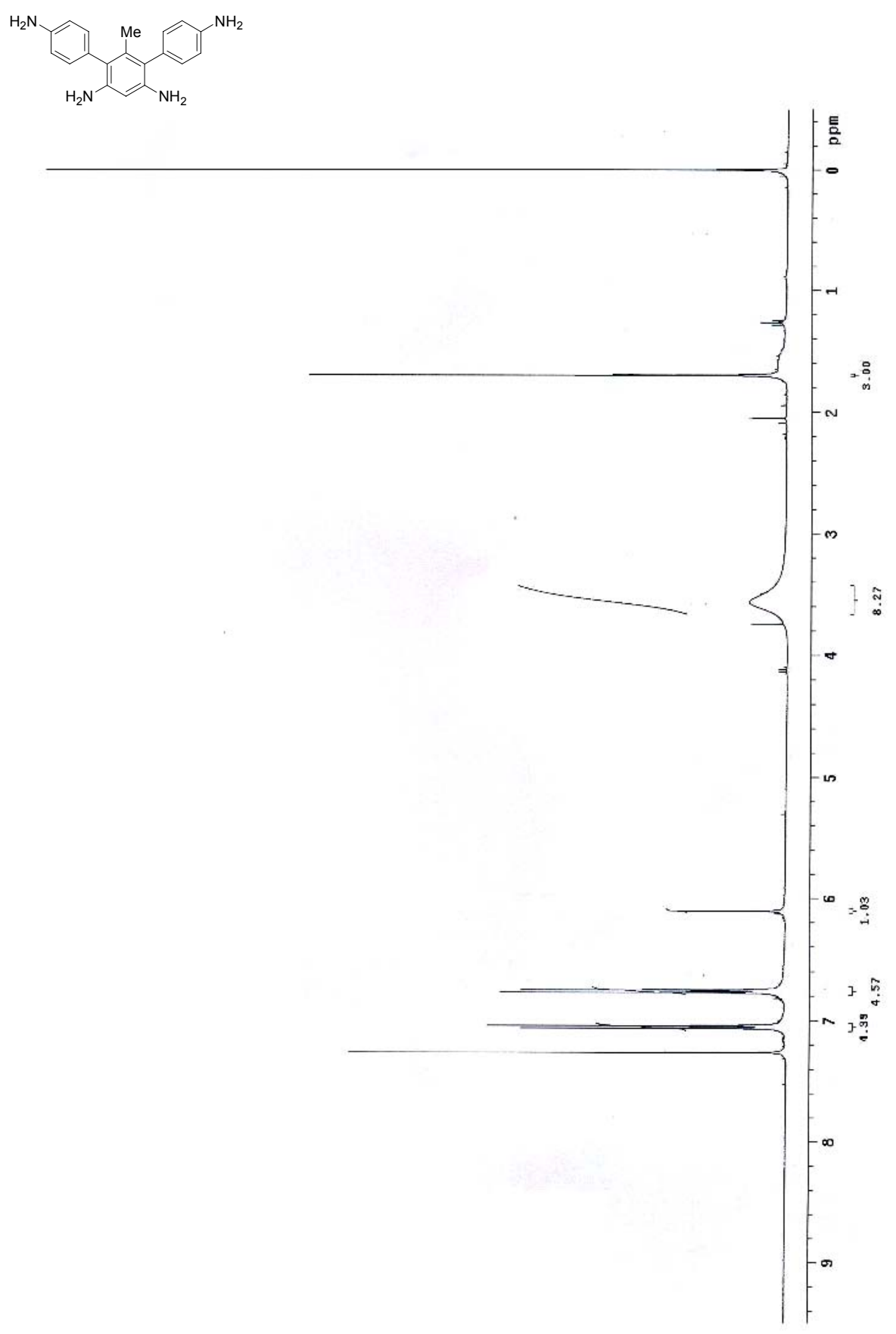



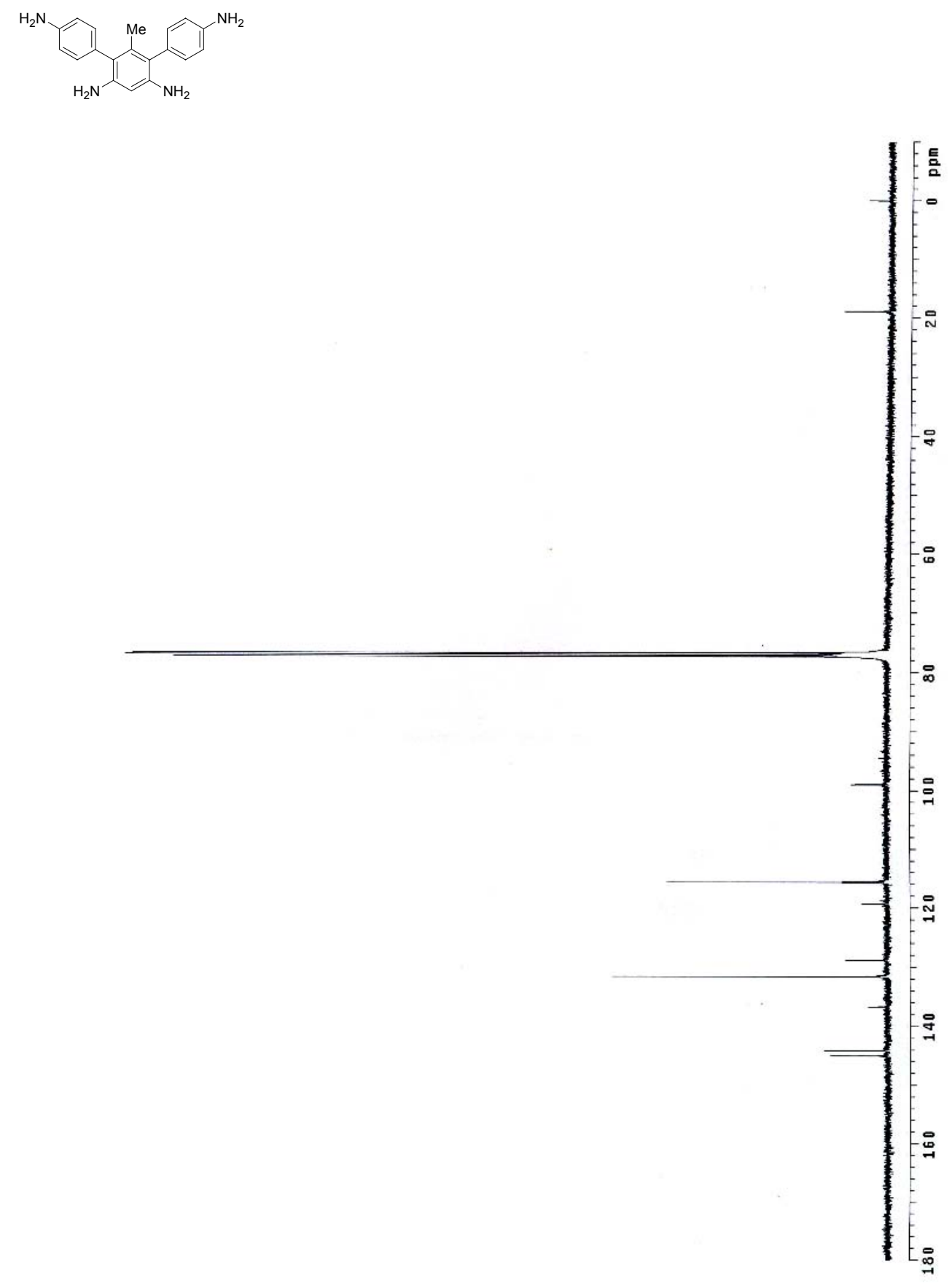


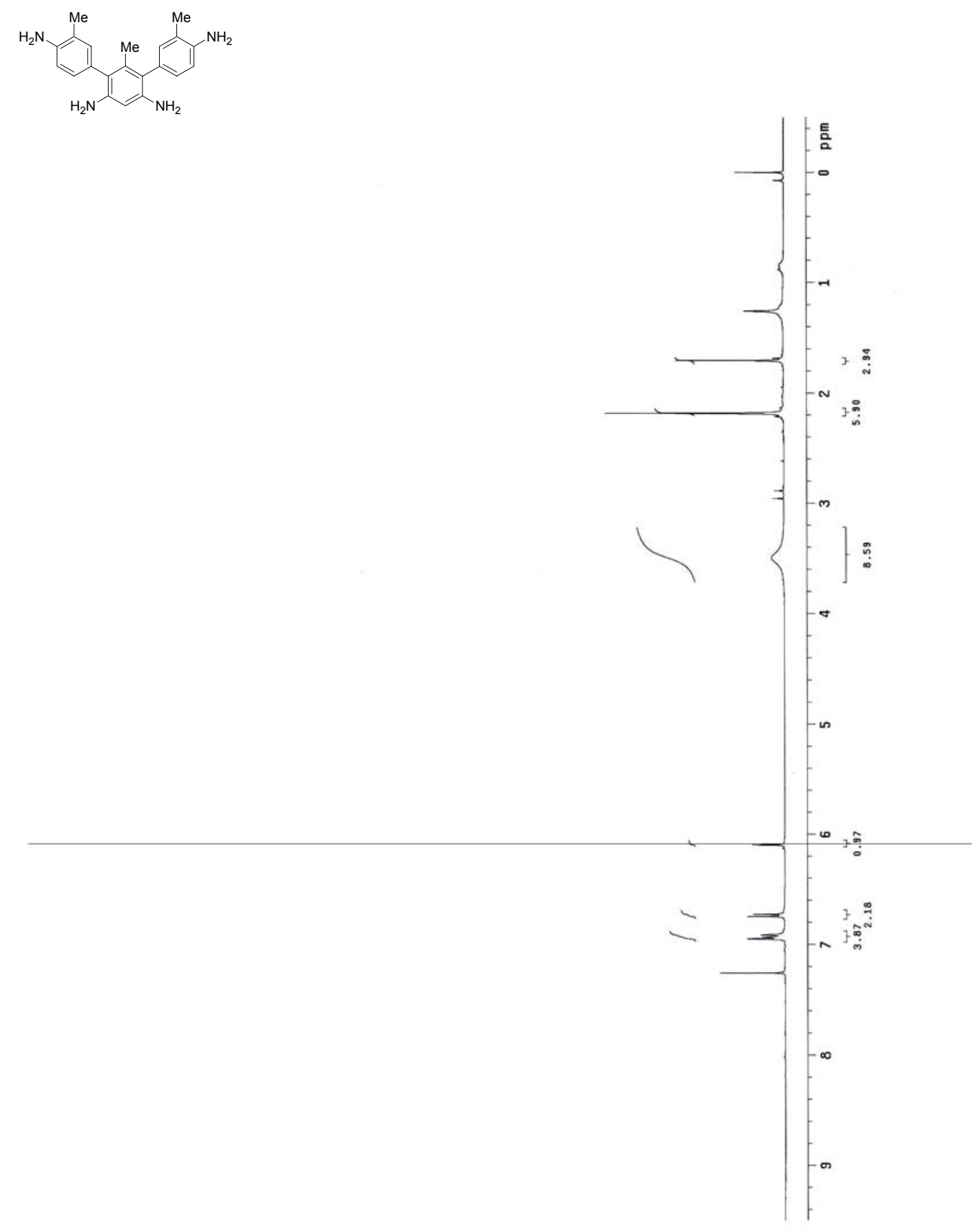



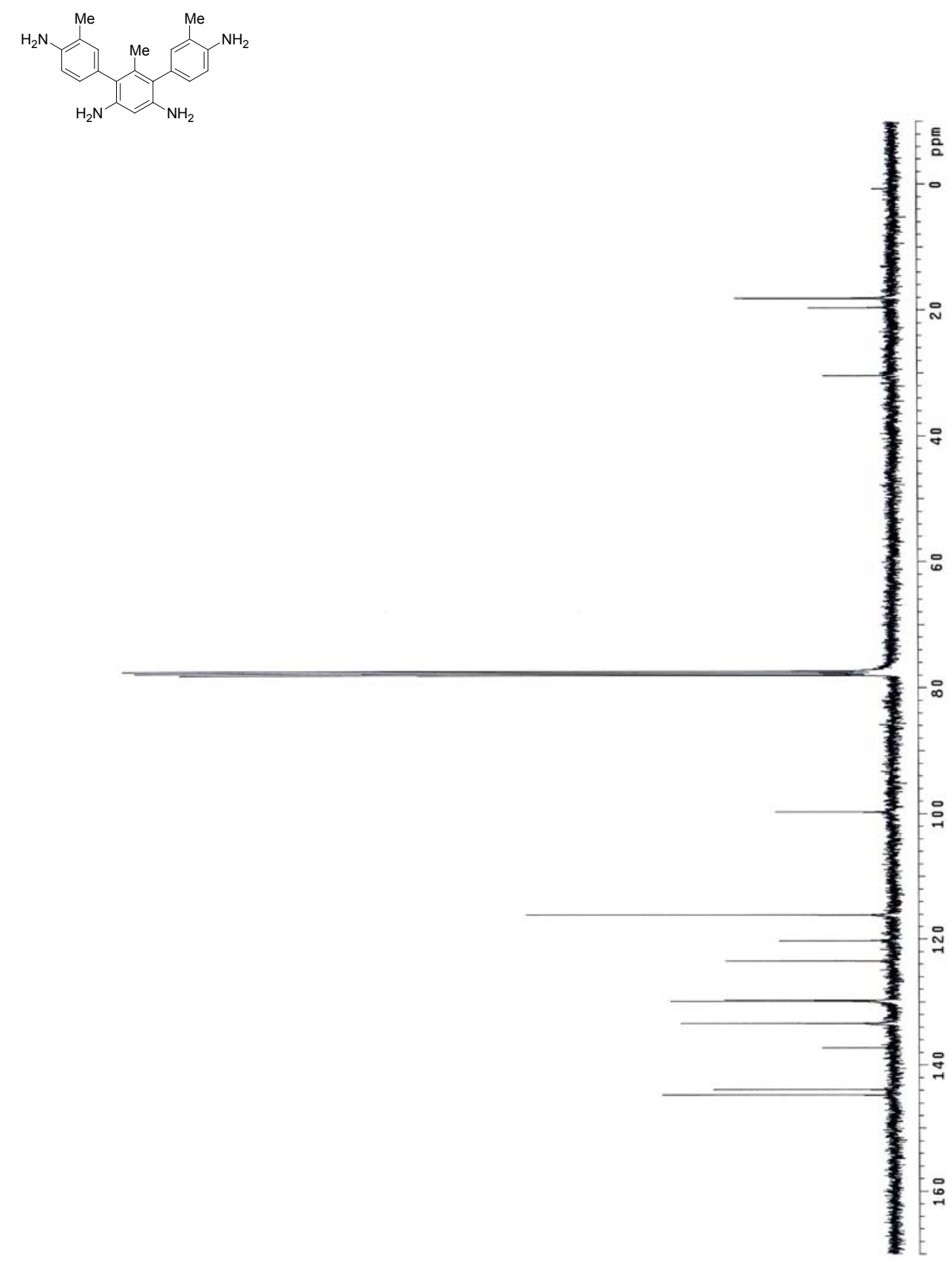

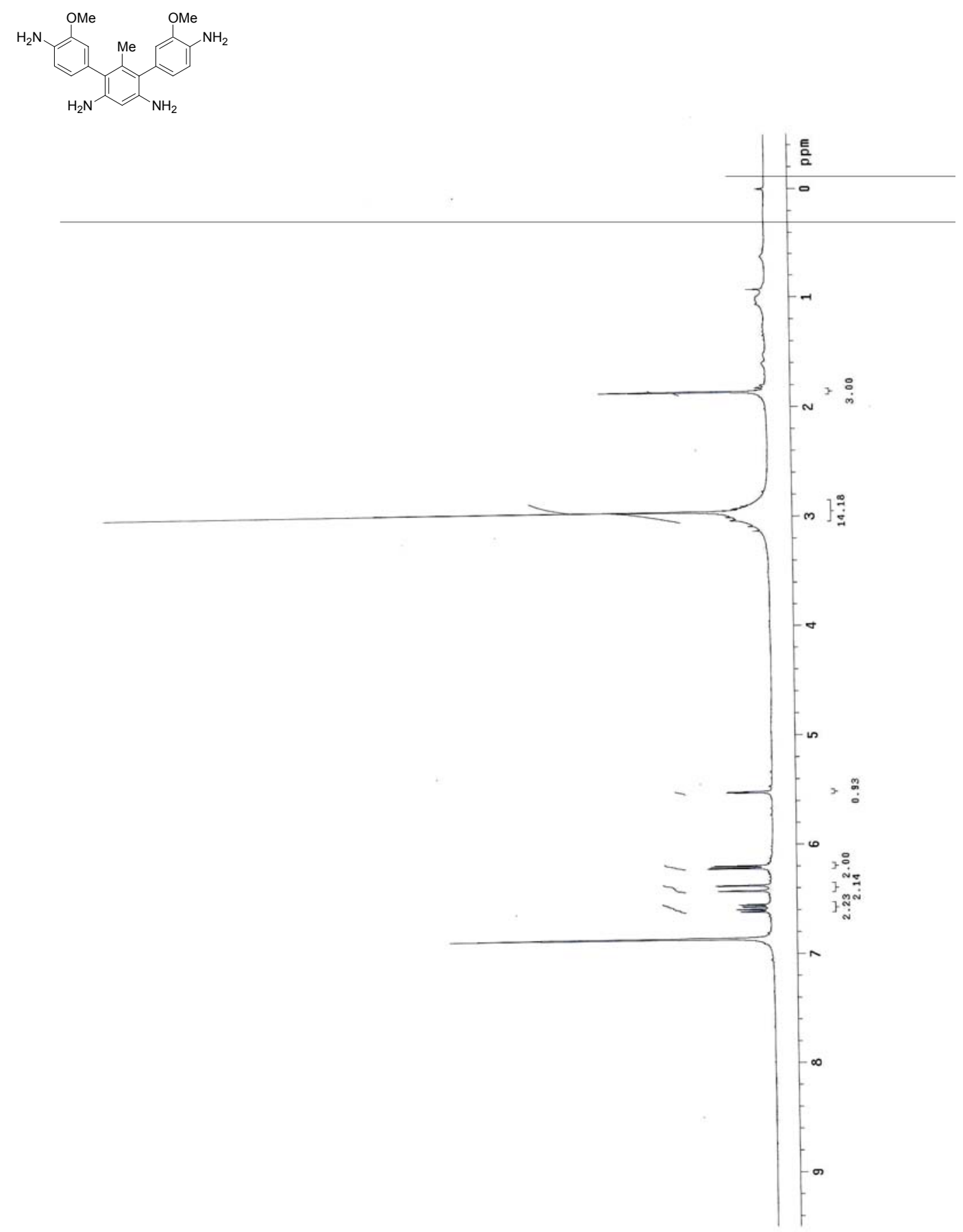

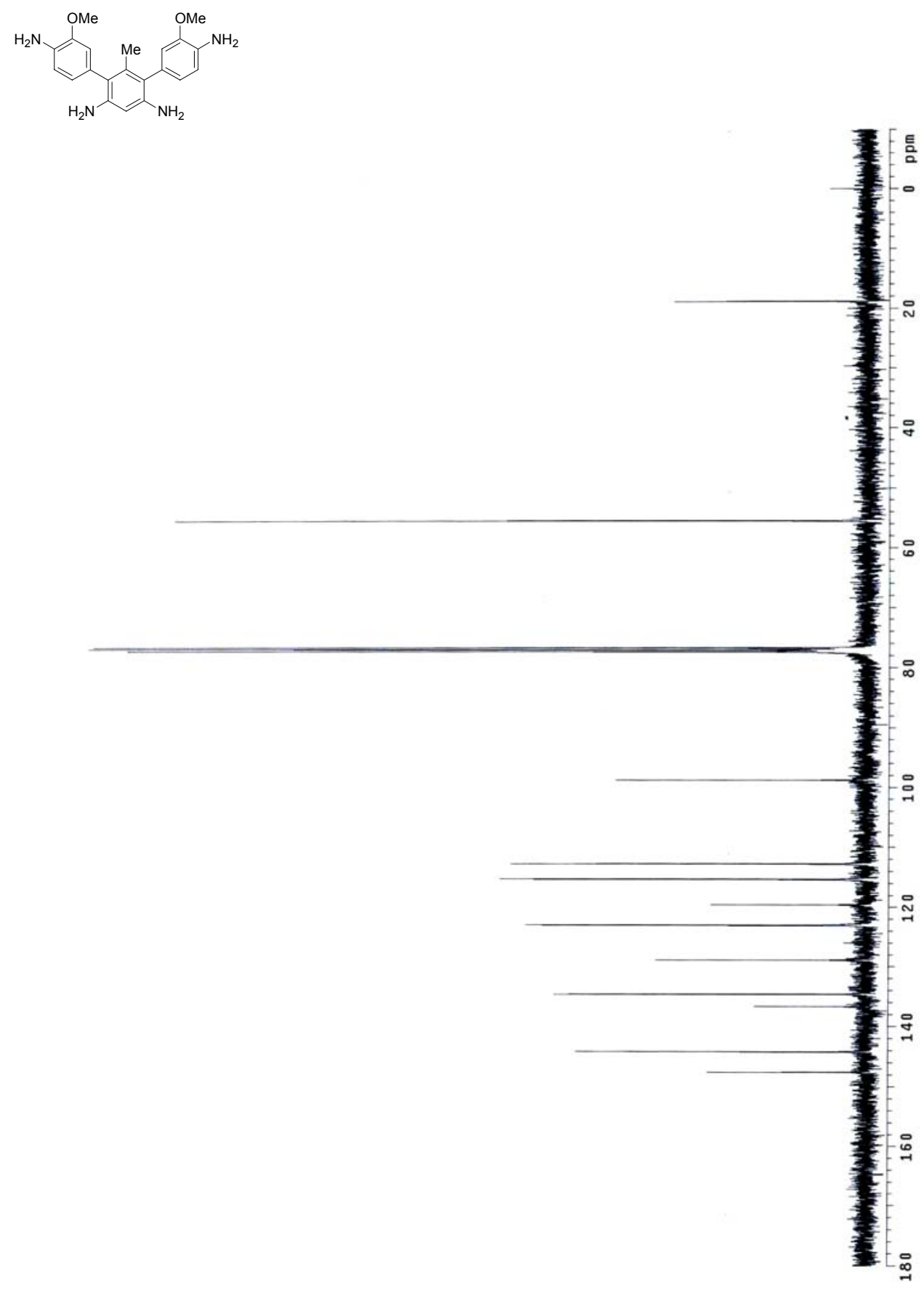

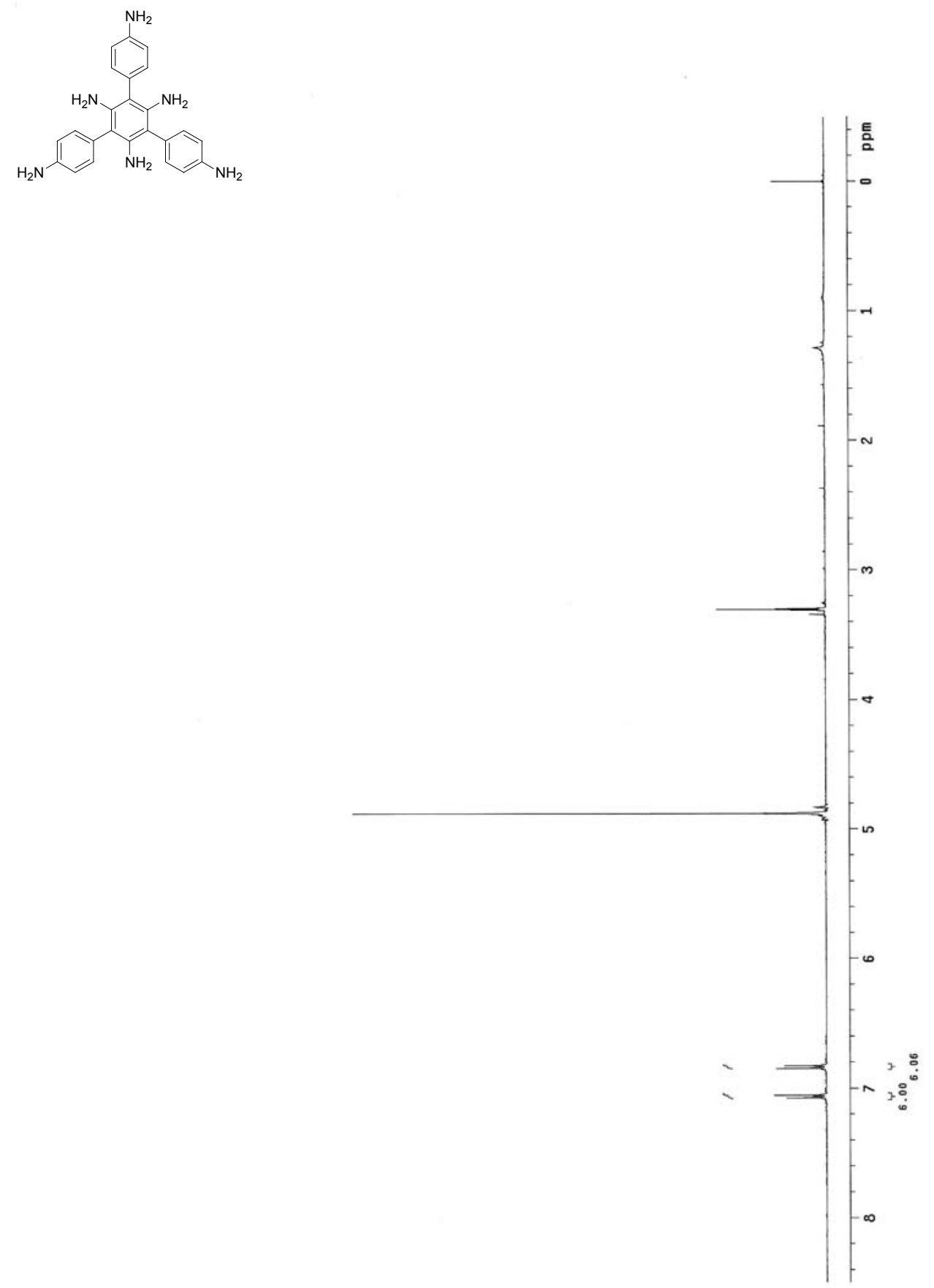

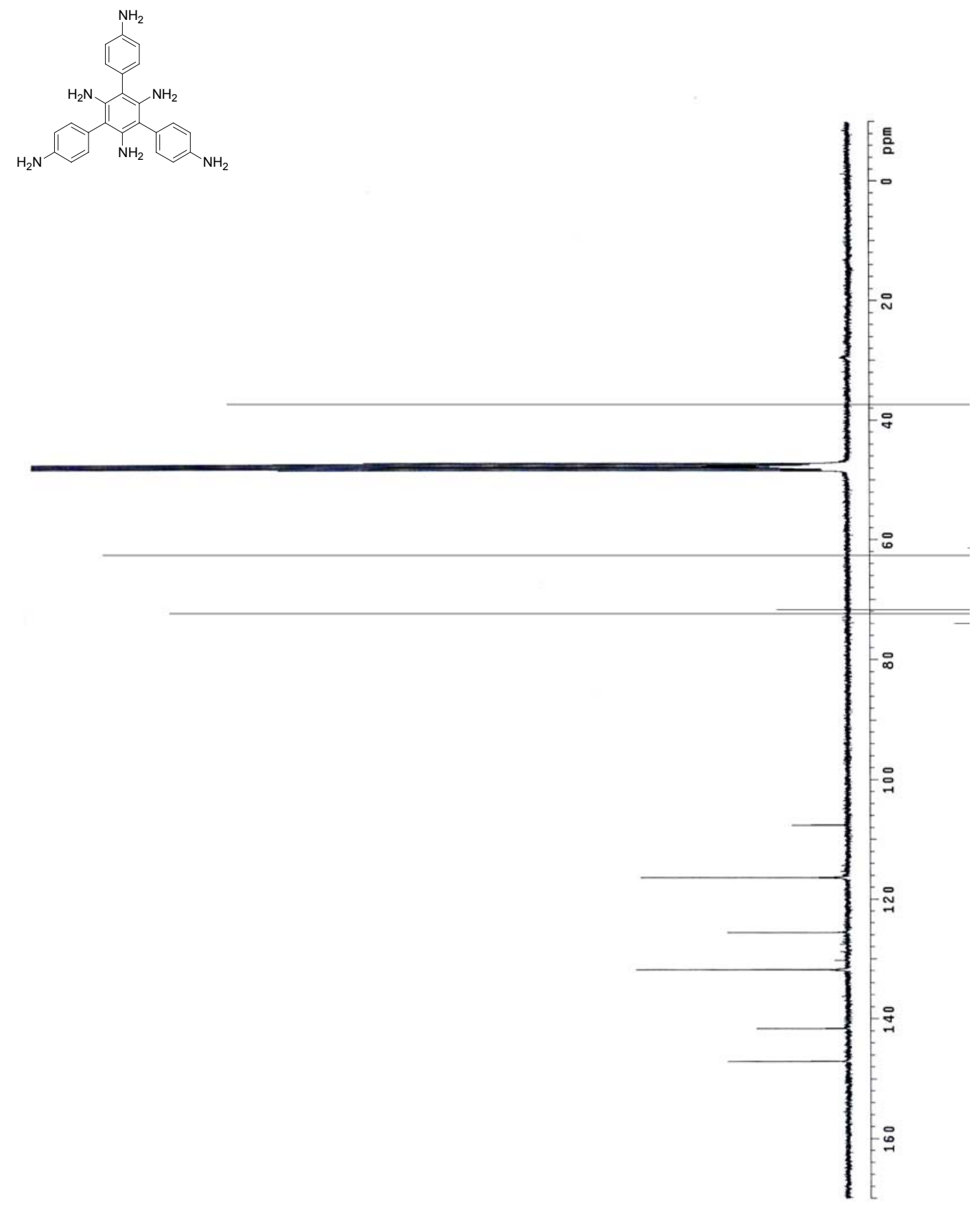


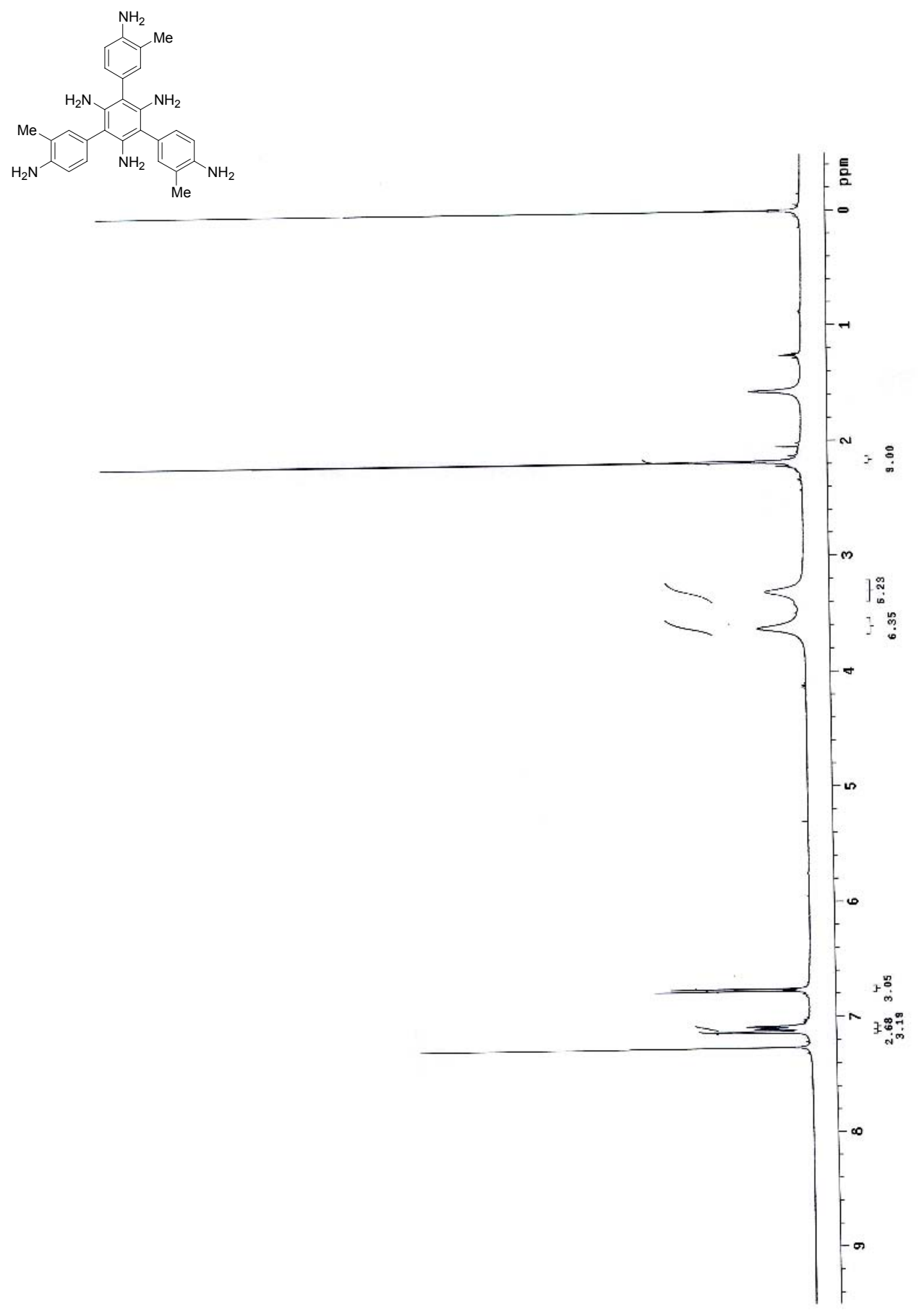



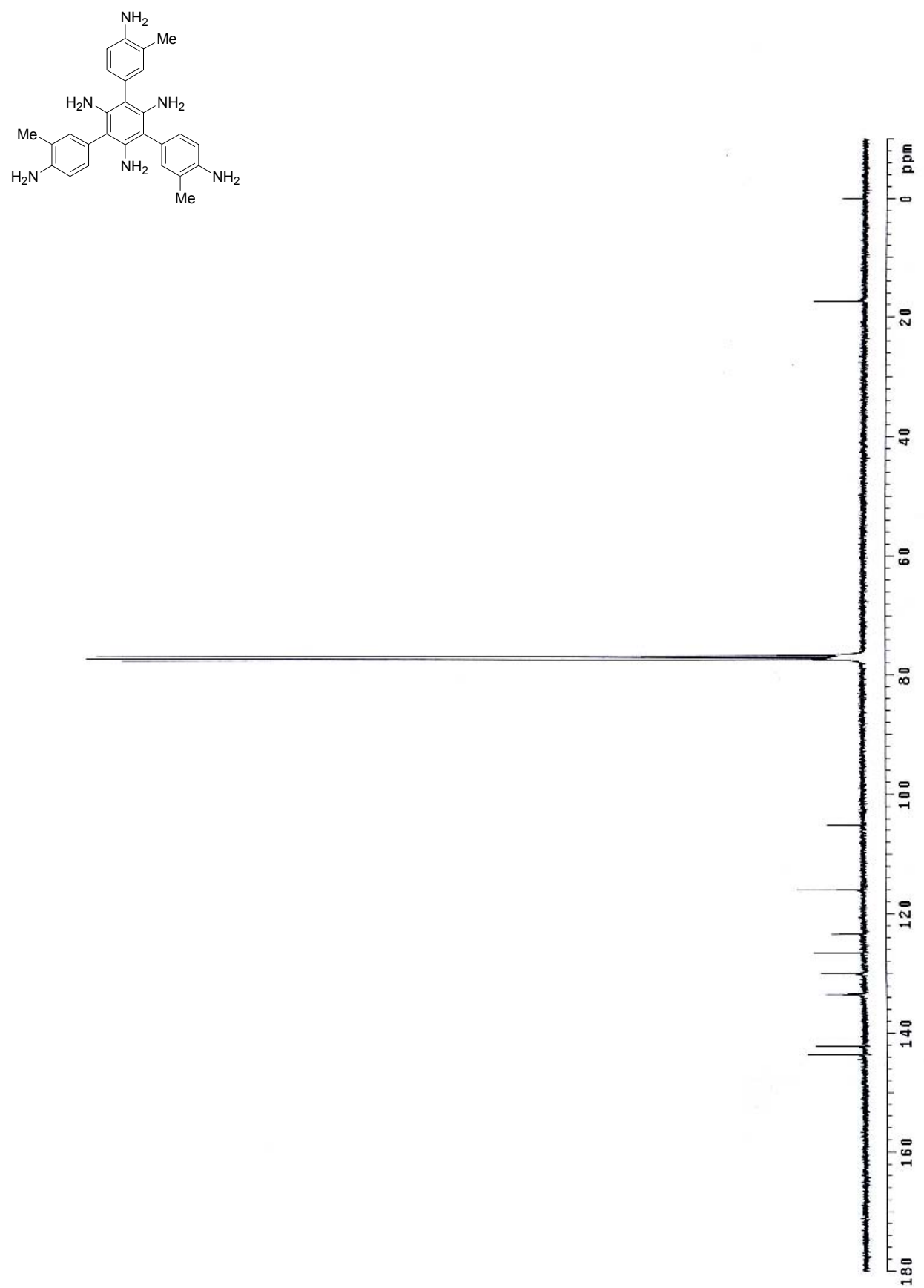


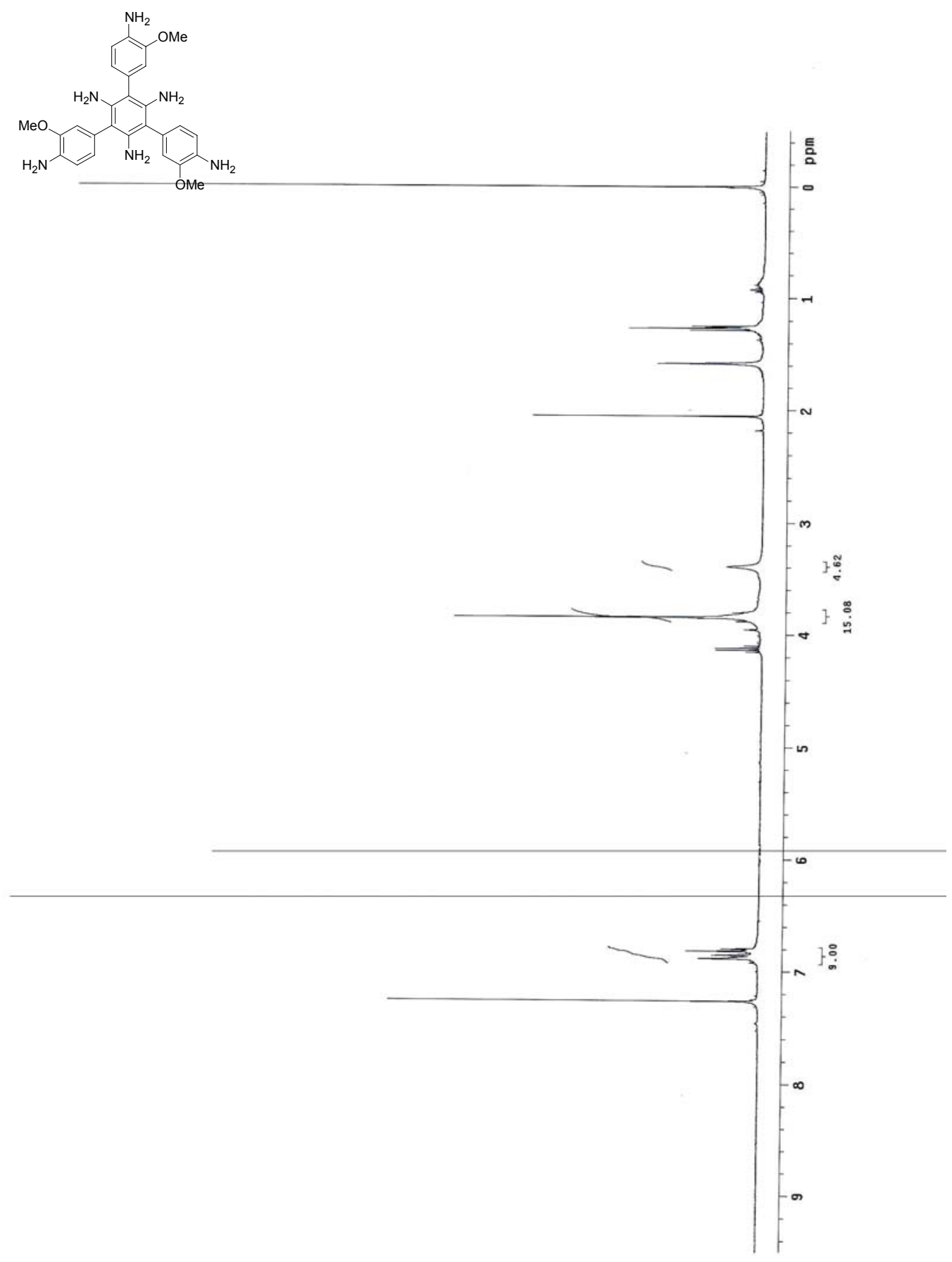



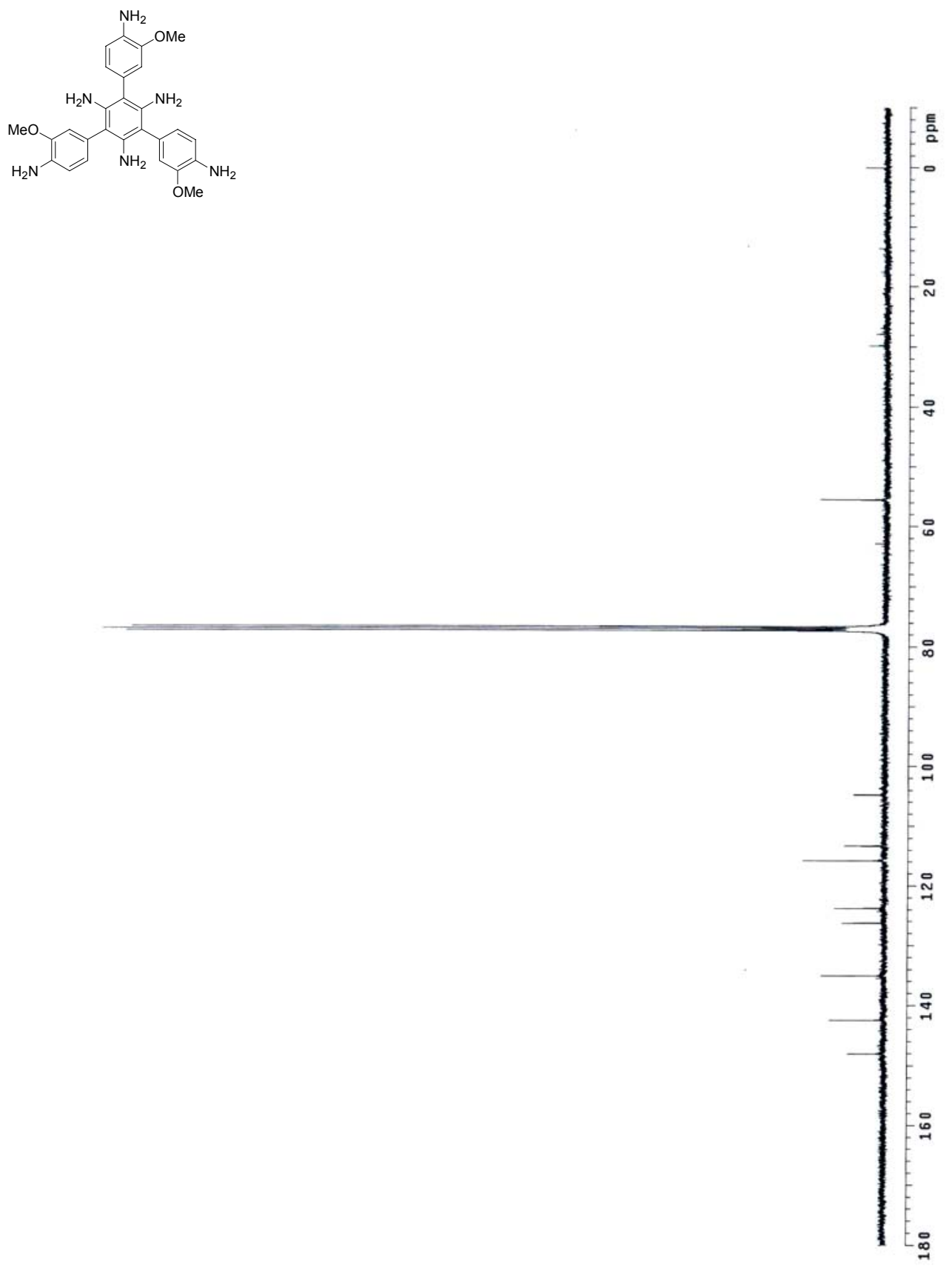